University of Montana

ScholarWorks at University of Montana

Numerical Terradynamic Simulation Group

Publications

Numerical Terradynamic Simulation Group

2000

\title{
Parameterization and Sensitivity Analysis of the BIOME-BGC Terrestrial Ecosystem model: Net Primary Production Controls
}

\author{
Michael A. White \\ Peter Edmond Thornton \\ The University of Montana \\ Steven W. Running \\ University of Montana - Missoula \\ Ramakrishna R. Nemani
}

Follow this and additional works at: https://scholarworks.umt.edu/ntsg_pubs Let us know how access to this document benefits you.

\section{Recommended Citation}

White, M., P. Thornton, S. Running, and R. Nemani, 2000: Parameterization and Sensitivity Analysis of the BIOME-BGC Terrestrial Ecosystem Model: Net Primary Production Controls. Earth Interact., 4, 1-85, doi: 10.1175/1087-3562(2000)004<0003:PASAOT>2.0.C0;2.

This Article is brought to you for free and open access by the Numerical Terradynamic Simulation Group at ScholarWorks at University of Montana. It has been accepted for inclusion in Numerical Terradynamic Simulation Group Publications by an authorized administrator of ScholarWorks at University of Montana. For more information, please contact scholarworks@mso.umt.edu. 


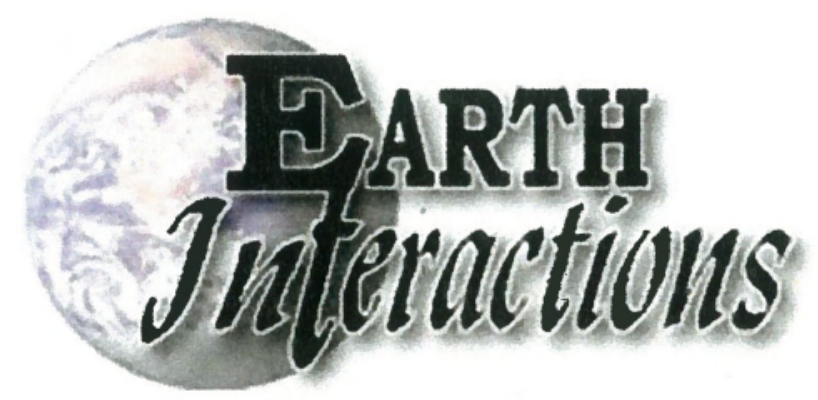

Copyright $\odot$ 2000. Paper 4-003: 38,228 Words, 5 Figures, 20 Tables. http://EarthInteractions.org

\title{
Parameterization and Sensitivity Analysis of the BIOME-BGC Terrestrial Ecosystem Model: Net Primary Production Controls
}

\section{Michael A. White,* Peter E. Thornton, Steven W. Running, and Ramakrishna R. Nemani}

Numerical Terradynamic Simulation Group, Missoula, Montana

Received 20 November 1999; accepted 21 June 2000.

\begin{abstract}
Ecosystem simulation models use descriptive input parameters to establish the physiology, biochemistry, structure, and allocation patterns of vegetation functional types, or biomes. For single-stand simulations it is possible to measure required data, but as spatial resolution increases, so too does data unavailability. Generalized biome parameterizations are then required. Undocumented parameter selection and unknown model sensitivity to parameter variation for larger-resolution simulations are currently the major limitations to global and regional modeling. The authors present documented input parameters for a process-based ecosystem simulation model, BIOMEBGC, for major natural temperate biomes. Parameter groups include the following: turnover and mortality; allocation; carbon to nitrogen ratios $(\mathrm{C}: \mathrm{N})$; the percent of plant material in labile, cellulose, and lignin pools; leaf morphology; leaf conductance rates and limitations; canopy water interception and light extinction; and the percent of leaf nitrogen in Rubisco (ribulose bisphosphate-1,5-carboxylase/oxygenase) (PLNR). Using climatic and site de-
\end{abstract}

* Corresponding author address: Dr. Michael A. White, NTSG, School of Forestry, University of Montana, Missoula, MT 59812.

E-mail address: mike@ntsg.umt.edu 
Earth Interactions - Volume 4 (2000) - Paper No. 3 - Page 2

scription data from the Vegetation/Ecosystem Modeling and Analysis Project, the sensitivity of predicted annual net primary production (NPP) to variations in parameter level of $\pm 20 \%$ of the mean value was tested. For parameters exhibiting a strong control on NPP, a factorial analysis was conducted to test for interaction effects. All biomes were affected by variation in leaf and fine root C:N. Woody biomes were additionally strongly controlled by PLNR, maximum stomatal conductance, and specific leaf area while nonwoody biomes were sensitive to fire mortality and litter quality. None of the critical parameters demonstrated strong interaction effects. An alternative parameterization scheme is presented to better represent the spatial variability in several of these critical parameters. Patterns of general ecological function drawn from the sensitivity analysis are discussed.

KEYWORDS: Biogeochemical processes; Plant ecology; Land/atmosphere interactions

\section{Introduction and background}

Terrestrial net primary production (NPP, $\mathrm{g} \mathrm{m}^{-2}$ ), equal to gross primary production minus autotrophic respiration, represents the carbon available for plant allocation to leaves, stems, roots, defensive compounds, and reproduction and is the basic measure of biological productivity. Tree growth, forage available for grazing, food production, fossil fuel production, and atmospheric $\mathrm{CO}_{2}$ levels are all strongly controlled by NPP. Accurate quantification of NPP at local to global scales is therefore central topic for carbon cycle researchers, foresters, land and resource managers, and politicians. For recent or current NPP estimates, satellite remote sensing can be used (e.g., Potter et al. 1993) but for research investigating pre1970s time periods or future climate scenarios, simulation models are required.

Models have been used to simulate regional water and carbon cycles under current and historical climates (Nemani et al. 1993; Running 1994), soil carbon dynamics (Motovalli et al. 1994), effects of nitrogen saturation (Aber et al. 1997), and the location of global carbon sources and sinks (Houghton et al. 1998; Randerson et al. 1997). Models can also be used to develop basic theoretical understandings of ecosystem function that cannot be tested with field methods (Churkina and Running 1998; Schimel et al. 1996). Perhaps most importantly, models are used to address the political and management need for estimates of ecosystem responses to climate changes (Intergovernmental Panel on Climate Change 1995). In particular, as fossil fuel consumption exponentially increases atmospheric $\mathrm{CO}_{2}$ (Keeling 1994) there is a growing need to provide credible estimates of ecosystem storage or release of carbon (Hunt et al. 1996; Schimel et al. 2000). NPP is a common component of these modeling approaches.

Large-scale biogeochemical (BGC) modeling, the topic of this research, is a specific type of modeling that seeks to mechanistically represent ecosystem cycles of carbon, water, and nutrients at regional to global scales through an integrated consideration of biology and geochemistry. The simulated land surface is divided into grid cells described by vegetation type (land cover), slope, aspect, elevation, albedo, and soil depth and texture (e.g., from Zobler 1986) from which soil water 
holding capacity and water release properties may be calculated (Clapp and Hornberger 1978). Nitrogen deposition, $\mathrm{CO}_{2}$ concentration, and climate data (usually monthly or daily) describe the atmosphere. Mathematical equations representing an abstraction of reality are then used to simulate ecosystem cycles of carbon (assimilation and respiration), nitrogen (mineralization, immobilization, leaching, volatilization, and denitrification), and water (evaporation, transpiration, and runoff).

The theoretical basis for NPP predictions and other model processes is usually based on realistic laboratory or field research, yet this same model realism often translates to a seemingly endless proliferation of difficult to obtain driving inputs, or parameters. In some cases, parameters are measured for a particular study, but when left unconstrained by measurement, parameters can be used as tuning knobs capable of producing a wide range of outputs. We feel that for these reasons, parameter selection and documentation, not model theory, are the main factors currently limiting the accuracy and believability of global and regional model simulations. As Aber (Aber 1997) stated: "ALL of the parameters used in the model should be listed, and ALL values for those parameters given, along with the references to the sources of those parameters." Aber also argued for complete descriptions of model structure and sensitivity. To address these and related concerns, our goals in this research are to

- provide an account of the source (or lack thereof) for parameters in BIOME-BGC, a commonly used terrestrial ecosystem process model, for major temperate biomes;

- assess the sensitivity of NPP to independent variation in every parameter;

- conduct a factorial sensitivity analysis of the most critical parameters;

- investigate patterns of ecosystem function revealed by the sensitivity analysis; and

- present a blueprint for an alternative parameterization scheme for critical parameters.

\section{Materials and methods}

\subsection{BIOME-BGC}

Using prescribed site conditions, meteorology, and parameter values, BIOMEBGC simulates daily fluxes and states of carbon, water, and nitrogen for coarsely defined biomes at areas ranging from $1 \mathrm{~m}^{2}$ to the entire globe. Plant physiological processes respond to diurnal environmental variation (Geiger and Servaites 1994), but BIOME-BGC uses a daily time step in order to take advantage of widely available daily temperature and precipitation data from which daylight averages of short wave radiation, vapor pressure deficit, and temperature are estimated (Thornton et al. 1997; Thornton and Running 1999). Nonlinear diurnal photosynthetic responses to radiation levels will not be captured by the use of daylight average radiation, but models initially designed to operate at daily timescales may still be used to accurately represent short-term variation in carbon fluxes (Kimball et al. 1997b). 
BIOME-BGC simulates the development of soil and plant carbon and nitrogen pools; no input of soil carbon information or leaf area index (LAI, $\mathrm{m}^{2}$ leaf area per $\mathrm{m}^{2}$ ground area) is required. LAI controls canopy radiation absorption, water interception, photosynthesis, and litter inputs to detrital pools and is thus central to BIOME-BGC. Model structure is discussed by Thornton (Thornton 1998) and is available online (www.forestry.umt.edu/ntsg), and will not be presented here. Briefly, though, NPP is based on gross primary production simulated with the Farquhar photosynthesis model (Farquhar et al. 1980) minus maintenance respiration [calculated as a function of tissue nitrogen concentration (Ryan 1991)] and growth respiration (a constant fraction of gross primary production). Theory and applications of BIOME-BGC and its predecessor, FOREST-BGC, are widely available (e.g., Hunt et al. 1996; Kimball et al. 1997b; Kimball et al. 1997c; Running 1994; Running and Coughlan 1988; Running and Gower 1991; Running and Hunt 1993; Running and Nemani 1991; White et al. 1999).

In BIOME-BGC, 34 parameters within several main categories are used to distinguish separate biomes. 1) Turnover and mortality parameters are used to describe the portion of the plant pools that are either replaced each year or removed through fire or plant death. 2) The allocation of photosynthetically accumulated carbon to leaf, stem, and root pools is controlled by a series of allometric parameters. 3) Carbon to nitrogen ratios define nutrient requirements for new growth, plant respiration rates, photosynthetic capacity, and litter quality. 4) The percentage of lignin, cellulose, and labile material in fine roots, leaves, and dead wood controls litter recalcitrance and influences decomposition rates. 5) Three morphological parameters control the distribution of LAI at the leaf and canopy level. 6) Several ecophysiological parameters are used to control rates of and limitations to leaf conductance. 7) Single parameters are used to control water interception, canopy radiation absorption, and the rate of carbon assimilation. Conceptually, the parameter groups describe biomes by rejecting excessive detail and unobtainable parameters while maintaining broadly significant vegetation descriptions.

\subsection{Parameterization}

For each parameter we conducted a literature search for each biome and calculated mean and standard deviation. There were two choices when assigning values: use the mean for each biome or conduct multiple comparison tests to group biome values together into statistically similar groups. Natural variability within biomes and, in some cases, limited sample sizes led the statistical approach to produce a homogeneous parameterization wherein biomes were remarkably indistinguishable. Since the ecological relevance of biome differences is well recognized (T. M. Smith et al. 1997) we chose the first option and did not test for statistically significant differences.

Data were usually available for evergreen needle leaf forest (ENF) and deciduous broadleaf forest (DBF), but in the grass literature, $\mathrm{C}_{4}$ data were rare and many authors reported "grasslands" without $\mathrm{C}_{3} / \mathrm{C}_{4}$ discrimination. We therefore parameterized a single grass biome. The $\mathrm{C}_{4}$ grass $(\mathrm{C} 4 \mathrm{G})$ is simulated with simple mechanisms to concentrate $\mathrm{CO}_{2}$ levels and to increase quantum yield efficiency. 
While some parameters were adequately treated for deciduous needle leaf forest (DNF), data for allocation parameters, the percent labile, cellulose, and lignin content in fine roots, litter, and dead wood, and leaf water stress parameters were lacking. With one exception related to photosynthesis, we applied the ENF values to DNF. Since data for shrubs were often sparse, defining multiple shrub categories, while perhaps ecologically appealing, was impractical. When shrub data were unavailable, we again generally assumed ENF values (see appendix A for exceptions).

\subsection{Sensitivity analysis}

\subsubsection{Inputs}

We used BIOME-BGC and the $0.5^{\circ} \times 0.5^{\circ}$ continental U.S. Vegetation/Ecosystem Modeling and Analysis Project (VEMAP) dataset (Pan et al. 1998; VEMAP 1995) to simulate NPP. VEMAP provided daily meteorology (T. G. F. Kittel et al. 2000, manuscripts in preparation; Kittel et al. 1997) for both preindustrial (1795-1894) and industrial (1895-1993) periods, soil texture and depth (Kern 1994; Kern 1995), and land cover (Küchler 1964; Küchler 1975). We reclassified the land cover into six classes: ENF, shrub, DNF [not represented at 0.5 resolution, distribution in Gower and Richards (Gower and Richards 1990) used to identify known areas], DBF, $\mathrm{C}_{3}$ grass (C3G), and C4G. To estimate VEMAP preindustrial nitrogen deposition, we first calculated a linear precipitation to deposition regression equation from a global 14-yr daily gridded meteorology dataset (Piper 1995) and total global preindustrial nitrogen deposition estimated from data in Holland et al. (Holland et al. 1997). We then applied the same relationship to the VEMAP preindustrial precipitation levels. We estimated industrial nitrogen deposition with $5^{\circ} \times 5^{\circ}$ MOGUNTIA (Dentener and Crutzen 1994; Zimmermann et al. 1989) predictions scaled to the 0.5 VEMAP resolution.

\subsubsection{Process}

Any given simulation followed a two-step procedure. First, using preindustrial meteorology, $\mathrm{CO}_{2}$ levels, and nitrogen deposition, soil carbon and nitrogen pools were initialized with BIOME-BGC simulations that terminated when equilibrium levels of net ecosystem carbon exchange were attained, typically 500-4000 yr. Second, the 1895-1993 period was simulated with increasing levels of $\mathrm{CO}_{2}$ [(VEMAP 1995), from ice core and atmospheric measurements]. Nitrogen deposition was increased from preindustrial to industrial (1990) levels at the same rate as $\mathrm{CO}_{2}$ increases.

\subsubsection{Independent variation sensitivity analysis}

We executed the sensitivity analysis with independent parameter variation as follows. We randomly selected 10 pixels for each of the six biomes (appendix B) and tested the effect of varying each parameter independently of other parameters by plus or minus a constant percent value. Ideally we would have varied the parameters within their measured range of variability, but because some parameters were based on a single value or used values from a different biome, such 
Earth Interactions - Volume 4 (2000) - Paper No. 3 - Page 6

an approach was impracticable. Instead we calculated one-tailed $95 \%$ confidence intervals and then calculated the average confidence interval (expressed as a percent of the mean value; only parameters based on at least two individual values were included). We varied parameters by the mean confidence interval (to one significant digit). We then arranged the parameters by the significance of their impact on NPP and identified the parameters most dominating BIOME-BGC predictions.

\subsubsection{Factorial sensitivity analysis}

For these limited parameters, we then adopted the suggestion of Henderson-Sellers and Henderson-Sellers (Henderson-Sellers and Henderson-Sellers 1996) and conducted a fractional factorial analysis [half-fraction approach in chapter 12 of Box et al. (Box et al. 1978)] to calculate main and interaction effects for two representative biomes. A factorial approach detects interacting effects of parameter variation, information that is impossible to obtain from varying parameters independently. In this case, the critical parameters were well documented and we used the measured range of parameter variability in the analysis. The range of variability used in factorial analysis is subjective and we initially used the standard deviation as the measure of variability. However, in the case where all critical parameters were set at levels expected to produce decreases in NPP, BIOMEBGC did not simulate biome development (i.e., the simulation "crashed"). We therefore used the standard error as the measure of variability. We calculated main and interaction effects for each of the 10 pixels per biome and then calculated the mean and confidence interval of the effects.

\subsection{Ecosystem synthesis and alfernative parameterization}

We then analyzed the results of the parameterization and sensitivity analysis for patterns of consistent biome and ecosystem function. Finally, we explored schemes to predict the spatial variability of critical parameters.

\section{Results and discussion}

\subsection{Parameterization}

Table 1 shows mean values for each parameter. Full parameter descriptions and a discussion of their role in BIOME-BGC, statistical information, species names, and citations are included in appendix A.

\subsection{Sensitivity analysis}

\subsubsection{Independent variation sensitivity analysis}

The mean confidence interval (expressed as percent of parameter mean) was $20 \%$. Results from the sensitivity analysis in which parameters were independently varied $\pm 20 \%$ show two key findings (Table 2 ): 1 ) only a small number of parameters consistently produced statistically significant differences in simulated 


\section{Earth Interactions - Volume 4 (2000) - Paper No. 3 - Page 7}

NPP and 2) the groups of important parameters were different between woody and nonwoody biomes.

Parameter C: $\mathrm{N}_{\text {leaf }}$ is the only one that exerted a significant control on NPP for all biomes (Table 2). For the woody biomes (ENF, shrub, DNF, and DBF), increasing C: $\mathrm{N}_{\text {leaf }}$ decreased NPP, while in $\mathrm{C} 3 \mathrm{G}$ and $\mathrm{C} 4 \mathrm{G}$, increased C: $\mathrm{N}_{\text {leaf }}$ had the opposite effect, increasing NPP. Thus, for woody biomes an increased leaf nitrogen investment and higher respiration cost was more than offset by increases in photosynthesis while in nonwoody biomes the opposite was true. PLNR (which strongly controls maximum rate of carboxylation), which had the largest effect for woody biomes but no significant effect in C3G and C4G (Table 2), is the main reason for this effect. Compared to the grasses, the woody biomes had low PLNR (Table 1); slightly increasing C: $\mathrm{N}_{\text {leaf }}$ reduced the amount of nitrogen available for investment in ribulose bisphosphate-1,5-carboxylase/oxygenase (Rubisco). In these biomes, the amount of Rubisco at mean C: $\mathrm{N}_{\text {leaf }}$ already limited the maximum rate of carboxylation and increasing $\mathrm{C}: \mathrm{N}_{\text {leaf }}$ created an even more severe photosynthetic limitation. Conversely, in the grass biomes, even at the mean minus $20 \%$ PLNR, Rubisco was still abundant and did not limit photosynthesis. By increasing $\mathrm{C}: \mathrm{N}_{\text {leaf }}$, grasses did not reduce photosynthetic capacity but they did reduce the cost of creating and maintaining leaves; a larger canopy and higher NPP were simulated. Increased leaf nitrogen investment in grasses simply created a nitrogen cost without increasing photosynthetic capacity.

In all biomes except DNF, higher C: $\mathrm{N}_{\mathrm{fr}}$ increased NPP by making more nitrogen available for investment in leaves. Unlike for C: $\mathrm{N}_{\text {leaf }}$, increases in C: $\mathrm{N}_{\text {fr }}$ do not have a negative physiological consequence for any biome and purely increase the pool of nitrogen available for investment in beneficial plant pools such as Rubisco. Beyond these two $\mathrm{C}: \mathrm{N}$ parameters, the significant parameters diverge for woody and grass biomes.

In woody biomes, three more parameters consistently impacted simulated NPP. First, increased new fine root carbon to new leaf carbon allocation (FRC: LC) diverted carbon from leaves into fine roots, but because BIOME-BGC does not mechanistically simulate root processes, it had no effect of increasing the efficiency of root nitrogen uptake. NPP therefore declined when FRC:LC was increased. Second, increased specific leaf area (SLA) resulted in higher LAI (LAI $=$ SLA $\times$ leaf carbon) without altering photosynthetic capacity, increasing water stress and reducing NPP. Third, increases in $g_{\text {smax }}$ reduced NPP by increasing water stress. The increases in potential leaf $\mathrm{CO}_{2}$ uptake caused by higher $g_{\text {smax }}$ were negated by increased depletion of soil water early in the growing season leading to stomatal down regulation of conductance later in the growing season. Parameters with a more limited effect included leaf and fine root turnover (LFRT, increased ENF NPP), $\mathrm{LAI}_{\text {all:proj }}$ (decreased ENF NPP), $W_{\text {int }}$ (decreased ENF NPP), and $k$ (decreased DBF NPP). In general, parameters decreasing LAI [high FRC: LC, $g_{\text {smax }}, \mathrm{LAI}_{\text {all:proj}}$, and $W_{\text {int }}$ (LAI data not shown)] decreased NPP, but LAI increases independent of increases in leaf nutrition (higher SLA) also decreased NPP.

In grass biomes, fire mortality (FM) and parameters relating to litter quality were far more important. Increasing FM, which increases nitrogen volatilization and reduces available mineral nitrogen, produced the largest NPP sensitivity for 
Earth Interactions - Volume 4 (2000) - Paper No. 3 - Page 8

C3G and $\mathrm{C} 4 \mathrm{G}$ but no discernable response for any woody biome. Increasing litter quality, as seen by significant effects from high levels of $F_{\text {cel }}, F_{\text {lab }}, L_{\text {cel }}$, and $\mathrm{L}_{\text {lab }}$, increased NPP, while reducing litter quality (high $\mathrm{FR}_{\mathrm{lig}}$ ) reduced NPP. Higher quality litter and fine roots decompose more rapidly than low quality material and increase the amount of nitrogen available for plant uptake.

\subsubsection{Factorial sensitivity analysis}

Because of similar behavior within woody and nonwoody biomes, we selected only two biomes for the half-fraction factorial analysis: ENF for woody and C3G for nonwoody. For ENF we selected C: $\mathrm{N}_{\text {leaf }}, g_{\text {smax }}$, PLNR, SLA, and C: $\mathrm{N}_{\mathrm{fr}}$. The variable FRC:LC was also important in the independent parameter analysis, but due to uncertainties in parameter variance and methodological difficulties inherent in obtaining the parameter (appendix A), we excluded FRC:LC from the design. For C3G, we used C:N $\mathrm{N}_{\text {leaf }}, \mathrm{FM}, \mathrm{FR}_{\text {cel }}$, $\mathrm{L}_{\text {cel }}$, and $\mathrm{C}: \mathrm{N}_{\mathrm{fr}}$.

In the half-fraction factorial design of $n$ parameters, $2^{n-1}$ simulations, or half the number of a full factorial design, are used (Box et al. 1978). Simulation time is halved with extremely small differences from the full factorial. Table 3 shows the design of the half-fraction [see Box et al. (Box et al. 1978) for details on constructing the table of contrast coefficients and calculating main and interaction terms].

Main effects are conceptually similar to the results from the independent parameter variation analysis but in this case were produced by runs with parameters set plus or minus their measured range of variability. Table 4 shows the main effect caused by increasing the parameter from the mean minus the standard error to the mean plus the standard error. All five main effects were statistically significant for both biomes. Consistent with results from the independent parameter analysis (Table 2), ENF was most affected by variation in PLNR while in C3G, FM had the largest effect. All main effects were at least 5.9 times larger than their standard errors.

Interaction effects (Table 5) show the difference between what would be expected by adding up the two main effects and what was actually observed from the interaction. For example, the ENF C: $\mathrm{N}_{\text {leaf }} \times$ PLNR interaction produced a 2.9 $\mathrm{g} \mathrm{m}^{-2}$ larger effect than would be expected from the sum of the C: $\mathrm{N}_{\text {leaf }}$ and PLNR main effects, indicating that the negative effects of increased C: $N_{\text {leaf }}$ were lessened by interacting increases in PLNR. The other two significant interaction effects were $g_{s \max } \times \mathrm{C}: \mathrm{N}_{\mathrm{fr}}$ and SLA $\times \mathrm{C}: \mathrm{N}_{\mathrm{fr}}$, both of which indicated that increased water stress caused by high $g_{s \max }$ or SLA dampened the increase in NPP caused by higher $C: \mathrm{N}_{\mathrm{fr}}$.

Interaction effects were often an order of magnitude less than main effects and the largest interaction term was 4.8 (4.7) times smaller than the smallest main effect in ENF (C3G). In C3G, no interaction term was significant and in ENF, only 3 out of 10 interactions were significant. To summarize, results from the factorial analysis presented in Tables 3-5 show that main effects are extremely dominant over interaction effects and that changes in simulated NPP can be approximated from the magnitude of the main effects in Table 4. 


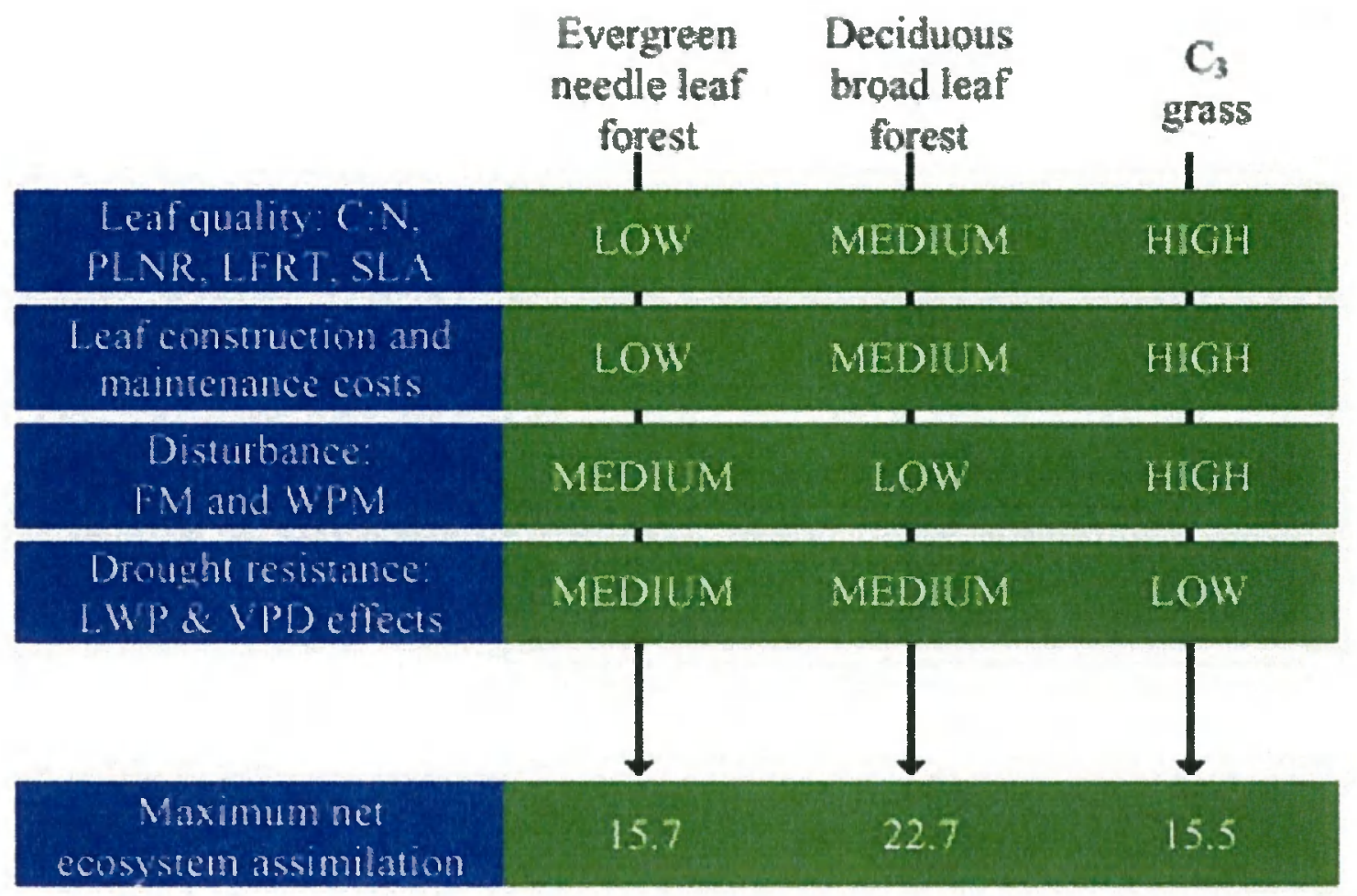

Figure 1. Conceptual chart of biome-level parameter groups and resulting maximum net ecosystem assimilation rates. Parameter categories (left boxes) are as follows. 1) Leaf quality is a function of $\mathrm{C}: \mathrm{N}_{\text {leaf }}$, the percent of leaf nitrogen in Rubisco (PLNR), LFRT (leaf and fine root furnover), and specific leaf area (SLA). Low C:N $\mathrm{N}_{\text {lear }}$, high PLNR, high LFRT, and high SLA create high photosynthetic capacity foliage. 2) Leaf costs represent the carbon and nitrogen costs to construct and maintain foliage. 3) Disturbance includes fire mortality (FM) and whole plant mortality (WPM). 4) Drought resistance represents plant ability to regulate stomatal conductance under vapor pressure deficit (VPD) and leaf water potential (LWP) stresses. Bottom panels show the average maximum net ecosystem assimilation rates ( $\mu \mathrm{mol} \mathrm{CO} \mathrm{C}^{-2} \mathrm{~s}^{-1}$ ) for each biome (Buchmann and Schulze 1999).

\subsection{Parameter associations and NPP limitations}

In a global survey of multiple vegetation types, Reich et al. (Reich et al. 1997; Reich et al. 1999) found strong linkages between SLA, leaf longevity, and leaf nitrogen concentration. In summary, high rates of net carbon assimilation at the leaf level $=$ high SLA $=$ short leaf longevity $=$ high leaf nitrogen concentration. Conceptually, the results showed that plants exist along a continuum from shortlived, high productivity to long-lived, less productive foliage. Here, even though we considered biome means and not continuous vegetation gradients, we found similar patterns in the three best-referenced biomes (Figure 1). 
Yet the parameter association identified by Reich et al. (Reich et al. 1997; Reich et al. 1999) as increasing instantaneous rates of assimilation do not necessarily result in increased assimilation at the ecosystem level (Figure 1). In all biomes, increased SLA increased LAI (data not shown) yet decreased NPP (Table 2) through a feedback from increased water stress. If NPP were limited by canopy assimilation capacity and not nitrogen availability (photosynthetic limitation), increasing leaf nitrogen would always increase NPP. For the woody biomes, this was true: increasing PLNR and reducing C: $\mathrm{N}_{\text {leaf }}$ increased NPP (Tables 2 and 4). For grasses, increased PLNR had little effect on NPP. Increasing grass C: $\mathrm{N}_{\text {leaf }}$, because of high grass PLNR and reductions in maintenance respiration calculated as a function of tissue nitrogen (Ryan 1991), increased NPP. Retranslocation was also lowest in grass (appendix A), suggesting that high photosynthetic investment may reduce the ability to recover nitrogen, further enhancing growth limitations. Thus, grasses appear to be limited by their foliage nutrition, both in terms of construction nitrogen required and respiration costs.

Field research appears to support these concepts. In their global survey of average maximum net canopy assimilation rates $\left(A_{\text {smax }}\right.$, total canopy, not per LAI), Buchmann and Schulze (Buchmann and Schulze 1999) found that the ordinal relationship among biomes was $\mathrm{DBF}>$ grass $\left(\mathrm{C}_{3}\right) \sim$ ENF. We speculate that the following general patterns, as illustrated in Figure 1, govern these results. In spite of optimal leaf nutrition, grass nitrogen limitations (to construct and maintain leaves), limited resistance to drought stresses, and high disturbance caused the low grass LAI found in Buchmann and Schulze (Buchmann and Schulze 1999). Thus a small grass canopy coupled with high photosynthetic capacity yields $A_{\text {smax }}$ values comparable to a large canopy of poor quality foliage in ENF. Longer growing seasons also compensate ENF for low quality foliage. With moderate controls on stomatal responses to water availability and moderate leaf nutrition and costs (Figure 1), DBF attained high LAI and the highest $A_{\text {smax }}$ of the three biomes (Buchmann and Schulze 1999).

\subsection{Reduction in parameter uncertainty}

While we have presented mean values, parameters should in reality vary spatially. For example, leaf longevity varies from about $2 \mathrm{yr}$ to over $10 \mathrm{yr}$ within the ENF (Figure 2, from appendix A). Cohesive patterns, such as decreasing leaf longevity at lower latitudes (Reich et al. 1995b), suggest the potential to spatially and realistically vary parameter levels. Not doing so may produce the correct spatially averaged NPP, but at any one location, over- or underestimation is likely. As a first attempt to reduce some of this uncertainty, we developed an approach to predict the spatial variability of C: $\mathrm{N}_{\text {leaf }}$, SLA, LFRT, and PLNR.

We used published equations (Yin 1993) to predict the spatial variation of $\mathrm{ENF}$ and DBF C: $\mathrm{N}_{\text {leaf }}$ based on climatic variation (climate from the VEMAP dataset). We then predicted SLA and LFRT (leaf longevity) with the equations relating $\mathrm{C}: \mathrm{N}_{\text {leaf }}$, SLA, and leaf longevity developed by Reich et al. (Reich et al. 1997) and calculated PLNR based on the spatial variation of SLA and C: $\mathrm{N}_{\text {leaf }}$ (see appendix A for PLNR equation).

Mean values between the spatial method presented here and the data pre- 


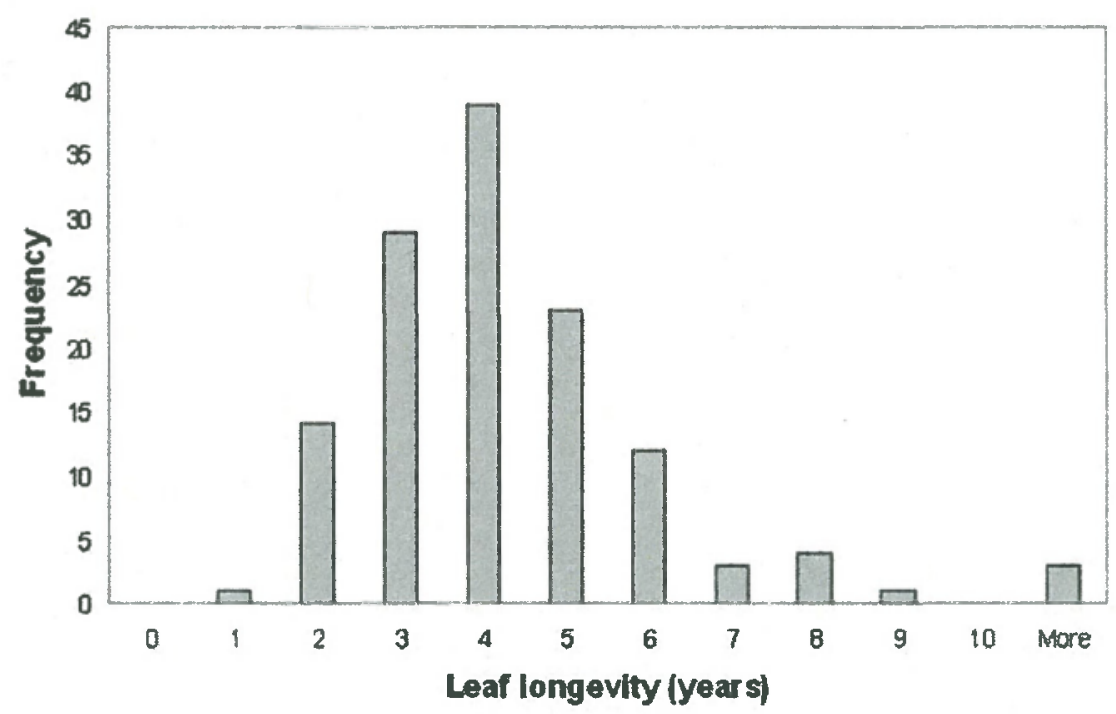

Figure 2. Frequency histogram of ENF leaf longevity. Data from appendix A.

sented above (Table 1) were generally similar for the ENF, but not for the DBF (Table 6). For ENF, mean C: $N_{\text {leaf }}$, SLA, leaf life span, and PLNR were all slightly higher in the spatial prediction (Figure 3 ) than in the single value per biome approach. Spatial patterns in Figure 3 were due entirely to initial C: $\mathbf{N}_{\text {leaf, }}$ which peaked in warm and cold climates (Minnesota and the far South) and reached a minimum in moderate climates. Large sections of the country were at approximately the same value for all four parameters. While the spatial prediction method produced mean values roughly comparable to the single value per biome approach, spatial patterns were troubling. For example, the methods in Yin (Yin 1993) predicted low nitrogen concentration in warm climates that in turn produced high leaf longevity in the south (Figure 3). Yet southern pine forests are known to have short leaf longevity (Reich et al. 1995b).

Mean DBF values showed significant differences between the two methodologies. In the spatial prediction (Figure 4), C: $\mathrm{N}_{\text {leaf }}$ was much higher than in the single value per biome approach. Consequently, the mean leaf life span was 13 months, more than twice as long as the leaf longevity predicted by the phenology subroutine (White et al. 1997) used in BIOME-BGC (Table 6). DBF SLA and PLNR were also low in the spatial prediction (Figure 4 and Table 6).

The prediction of very low SLA and numerous leaf life spans greater than $2 \mathrm{yr}$ in the DBF and incorrect patterns of leaf longevity in the ENF are not acceptable results. We suspect that the range of climates used in Yin (Yin 1993) may not have been representative of the entire climatic range of the biome, thus leading to high C: $\mathrm{N}_{\text {leaf }}$ predictions in inappropriate areas. Note that in the northeastern United States, where many of the studies in Yin (Yin 1993) were concentrated, DBF leaf life spans, $\mathrm{C}: \mathrm{N}_{\text {leaf }}$, and SLA were in a more normal range. These patterns illustrate both the exciting potentials of this methodology and its potential pitfalls. We believe that while this approach is conceptually superior to 


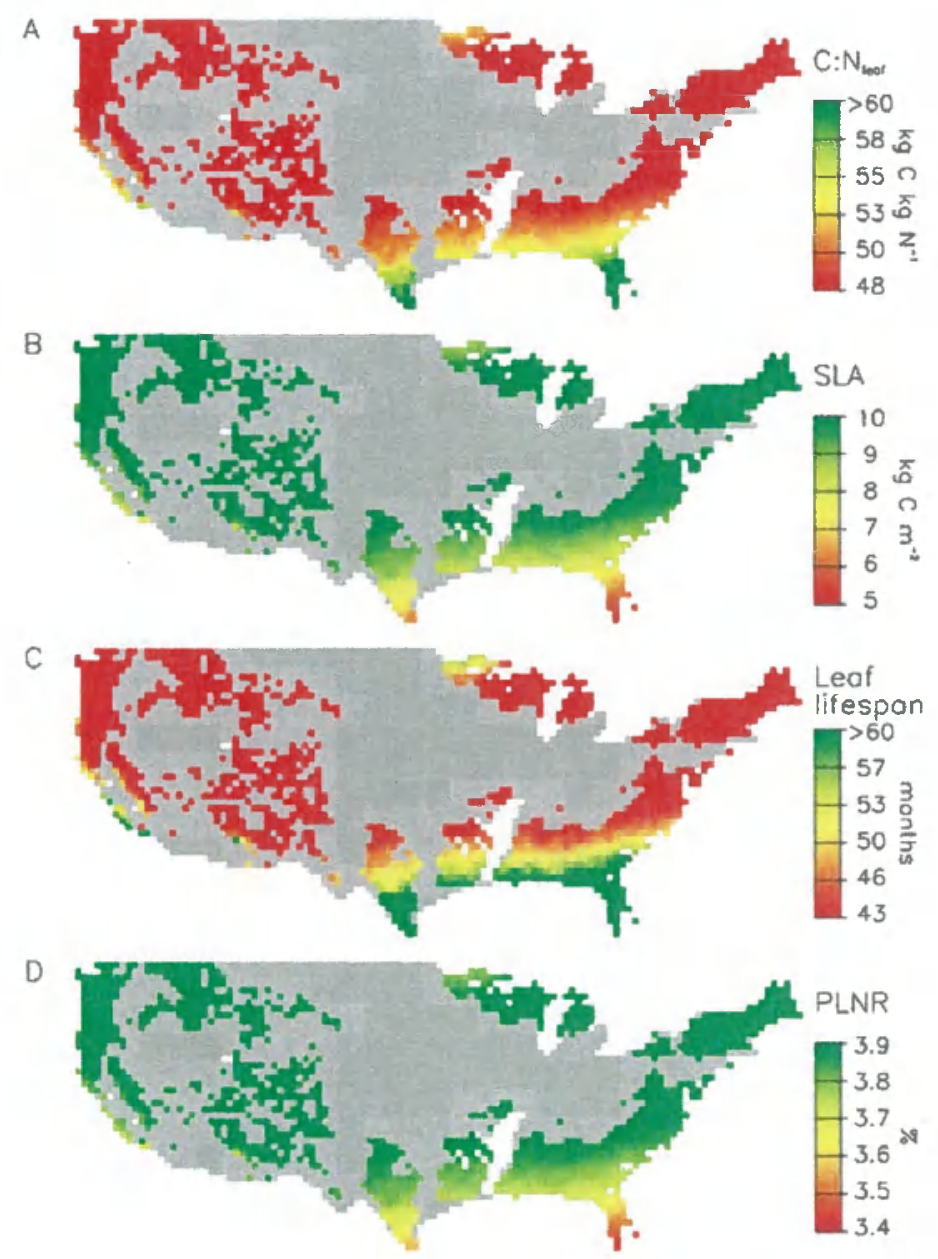

Figure 3. ENF parameter variation in the continental United States: (a) C:N $N_{\text {leat }}(\mathrm{kg}$ $C \mathrm{~kg} \mathrm{~N}^{-1}$ ), (b) specific leaf area (SLA, $\mathrm{kg} \mathrm{C} \mathrm{m}^{-2}$ ), (c) leaf life span (months), and (d) the percent of leaf nitrogen in Rubisco (PLNR, dimensionless). Gray areas do not contain ENF in the VEMAP dataset.

using single values per biome, its adoption with the existing equations is premature. Future work should focus on predicting a more global distribution of $\mathrm{C}$ : $\mathrm{N}_{\text {leaf }}$ (appendix A; Reich et al. 1997; Reich et al. 1999) from site climate. Most studies do not publish climatic data, but with a global network of meteorological stations (Piper 1995) and a microclimate simulator (Glassy and Running 1994; Kimball et al. 1997a; Thornton and Running 1999), it is possible to estimate climate for any site.

The remaining critical parameters are more problematic. The parameter $\mathrm{C}$ : $\mathrm{N}_{\mathrm{fr}}$ appears to follow consistent patterns with climate (Yin and Perry 1991), again suggesting the possibility of using site climate to predict C:N. However, C: $\mathrm{N}_{\mathrm{fr}}$ calculation also relies on root diameter data, which are not currently available at large scales. We suggest that FRC:LC will be best obtained by calculation, not measurement. Given that the majority of other parameters are 1) better con- 


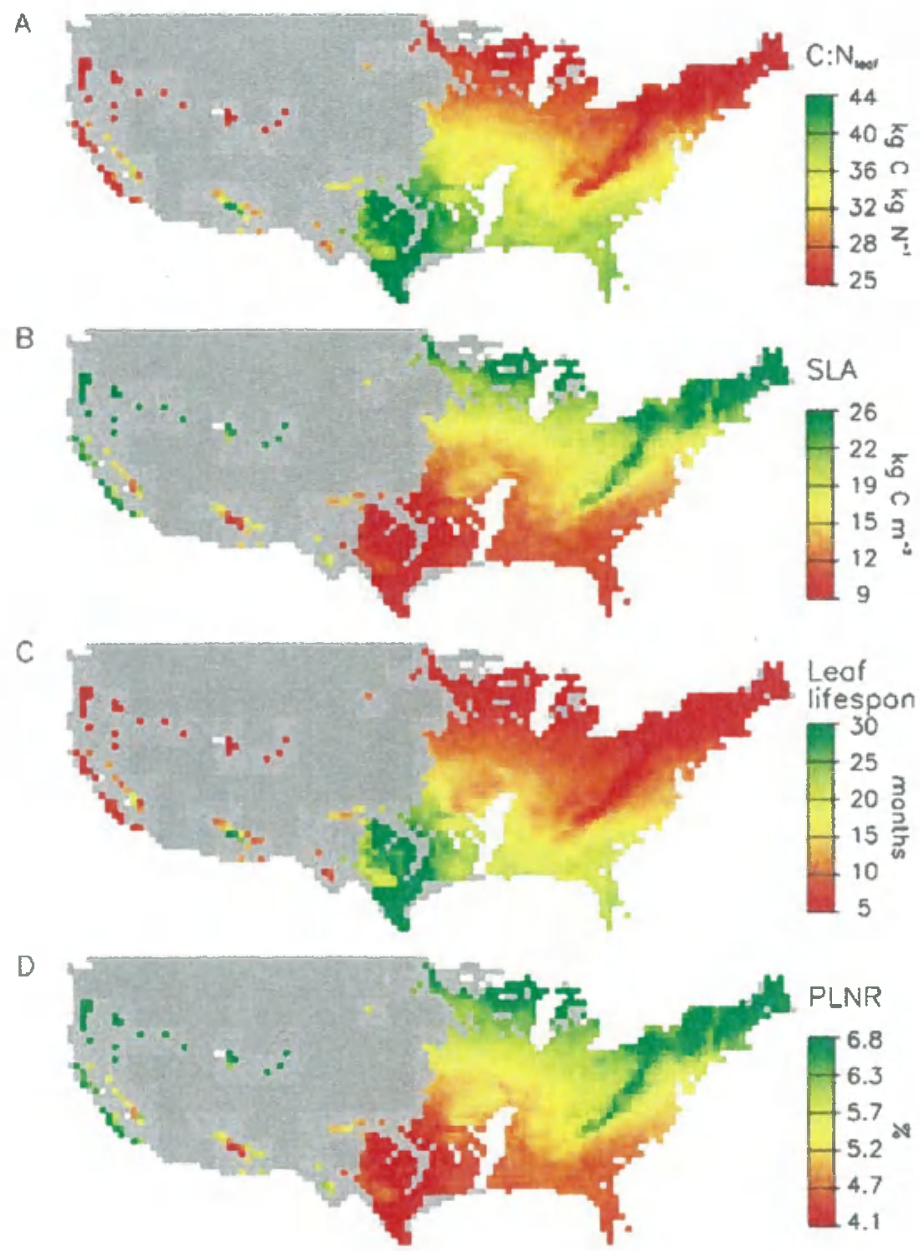

Figure 4. DBF parameter variation in the continental United States. Panels as in Figure 3. Gray areas do not contain DBF in the VEMAP dataset.

strained, 2) exert minor control over NPP, 3) could be fairly easily measured for a given site $\left(C: N_{\mathrm{fr}}\right)$, or 4$)$ could be resolved with the preceding scheme, it may be possible to solve for FRC:LC with an inverse method based on known values of total ecosystem exchange from eddy covariance methods (Baldocchi et al. 1996; Goulden et al. 1996). Currently, eddy covariance techniques are not adequate for this approach, but future improvements may make it possible. Reduction in FM uncertainty will require the development of a spatially variable global fire mortality dataset.

\subsection{Suspicious results}

While most of the results and interpretations presented above are fairly straightforward and follow logically from physiological and physiographic concepts, some of the results and parameterizations may be artifacts of model design or field data. 
Increased C: $\mathrm{N}_{\mathrm{fr}}$, for example, reduces root nitrogen requirements and diverts nitrogen to increased photosynthetic capacity (higher NPP for most biomes; Table 2). Similarly, high FRC:LC diverts carbon to fine roots and away from LAI. Nitrogen and carbon are in essence allocated to the roots without any assimilation benefit from increased investment. In a purely mathematical sense, plants without roots will produce the highest NPP. However, it is well known that the vast majority of flowering plants maintain an active root system requiring carbon and nitrogen and we therefore include these costs in the model design. Adding the model complexity required to accurately model root density and distribution, ion gradients, mycorrhizal associations, etc. is not practical for large-scale ecological models, but should be considered for stand-specific efforts.

Reducing $g_{\text {smax }}$ from $0.006 \mathrm{~m} \mathrm{~s}^{-1}$ increased NPP for every biome except C4G (Table 2), indicating that most biomes have nonoptimal $g_{\text {smax }}$. Nonoptimal levels for other parameters are usually somehow offset (i.e., poor leaf nutrition in ENF produces low respiration rates). For $g_{s \max }$, it is not clear what compensates for the high water loss. While $0.006 \mathrm{~m} \mathrm{~s}^{-1}$ may represent the maximum possible $g_{\text {smax }}$ under nonlimiting environmental conditions, average growing season $g_{\text {smax }}$ may be more appropriate for ecosystem modeling. It is also possible that $g_{s \max }$ should also be reduced in the shaded canopy portion (Beadle et al. 1985; Kozlowski and Pallardy 1997; Oberbauer et al. 1987). Based on data showing $g_{s \max }$ reductions with leaf age (Field and Mooney 1983; Igboanugo 1996; Leverenz et al. 1982), it may further be realistic to reduce $g_{\text {smax }}$ as a function of LFRT. Finally, if more data were available, it is possible that $g_{s \max }$ would vary statistically between biomes. Future research should focus on establishing the most appropriate $g_{s \max }$ values for ecosystem modeling approaches.

\section{Conclusions and suggestions}

For the first time in the history of BIOME-BGC and its predecessor, FORESTBGC (Running and Coughlan 1988; Running and Gower 1991), we have produced a complete documentation of all model parameters and a comprehensive model sensitivity analysis for major natural temperate biomes. Major conclusions from the parameterization and sensitivity analysis include the following.

- Greatest NPP increases were created by 1) increasing leaf nitrogen content and nitrogen investment in Rubisco in woody biomes and by 2) reducing leaf nitrogen content and increasing nitrogen availability in nonwoody biomes.

- This suggests that productivity is photosynthetically limited for woody biomes and nitrogen limited for nonwoody biomes.

- Parameters varied logically such that biomes could not simultaneously have high productivity foliage, long leaf life span, low exposure to drought stresses, and low fire and mortality fluxes. Biomes tend to exist on a continuum from high quality foliage with a short and risky life span to low quality foliage with a long life and lower risk of fire and mortality.

- This research clearly shows why simplified NPP modeling approaches (Aber et al. 1996; Coops 1999) work well; for coarse time resolution 
growth simulations, a very limited number of critical physiological variables are responsible for most output variation.

Simulations in other climates may produce a different list of critical parameters. We speculate that higher precipitation in the wet Tropics may eliminate SLA and $g_{\text {smax }}$ from the list and add controls on the absorption of radiation, such as the light extinction coefficient. The sensitivity analysis should therefore be expanded to include a more globally representative climatic distribution.

Exploration of more appropriate $g_{s \max }$ values for modeling research and the implementation of mechanisms to regulate the efficiency of mineral nitrogen uptake as a function of root carbon and nitrogen investment should be future priorities. However, we believe that accurate predictions of the spatial distribution of several key parameters would produce the greatest reduction in the uncertainty of large-scale NPP simulations. We presented a blueprint for such an approach, but results were inconclusive. Further research on this topic is a critical priority.

Acknowledgments. We thank Alisa Keyser for editing the manuscript and Anna Sala, Lloyd Queen, and Don Potts for their critiques. Andy Pitman and an anonymous reviewer provided helpful comments. NASA Grant NAG531368 supported this research. Michael White was supported by the NASA Earth System Science Fellowship.

\section{Appendix A: Individual Parameter Values}

Values presented here were converted from their original units to BIOME-BGC units. We assumed that carbon was $50 \%$ of dry weight. For parameters based on multiple literature values, we include summary statistics (mean, standard deviation, number of values) and individual citations. In cases where data were unavailable for DNF and shrub biomes, we used ENF values (exceptions noted below).

\section{A.1 Turnover and mortality parameters}

Turnover refers to the percent of the carbon pool replaced each year (flux/mass) and is the inverse of the mean residence time (mass/flux). Mortality refers to the fraction of the carbon pool removed by plant death, either through whole plant mortality or fire mortality. Turnover and mortality parameters, with one exception, are relatively poorly documented parameters in BIOME-BGC and should be priorities for future work.

\section{A.1.1 Leaf and fine root turnover}

For all deciduous biomes, LFRT $\left(1 \mathrm{yr}^{-1}\right)$ is set to 1.0 , indicating that the entire leaf and fine root carbon pools are turned over every year. The rationale for linking leaf and fine root turnover is presented in Thornton (Thornton 1998). ENF LFRT data are compiled from extensive foliage production and biomass data and shows mean leaf longevity of $3.8 \mathrm{yr}$. Values presented here are leaf turnover values. Original data were in leaf longevity. The mean value used in the parameterization is calculated from the inverse of the mean leaf longevity. A different 
(and larger) value of leaf and fine root turnover is obtained by taking the mean of the values presented below.

\begin{tabular}{|c|c|c|}
\hline \multirow{2}{*}{$\begin{array}{l}\text { ENF } \\
\text { Abies amabilis }\end{array}$} & \multicolumn{2}{|c|}{ Mean $=0.26$, std dev $=0.15, n=129$} \\
\hline & 0.093 & $\begin{array}{l}\text { (Gholz et al. 1976; Grier and Milne 1981; Grier } \\
\text { et al. 1981) }\end{array}$ \\
\hline Abies amabilis & 0.047 & $\begin{array}{l}\text { (Gholz et al. 1976; Grier and Milne 1981; Grier } \\
\text { et al. 1981) }\end{array}$ \\
\hline Abies balsamea & 0.270 & (Baskerville 1965; Baskerville 1966) \\
\hline Abies balsamea & 0.270 & (Baskerville 1965; Baskerville 1966) \\
\hline Abies balsamea & 0.263 & (Baskerville 1965; Baskerville 1966) \\
\hline Abies balsamea & 0.270 & (Baskerville 1965; Baskerville 1966) \\
\hline Abies balsamea & 0.270 & (Baskerville 1965; Baskerville 1966) \\
\hline Abies balsamea & 0.278 & (Baskerville 1965; Baskerville 1966) \\
\hline Abies concolor & 0.244 & $\begin{array}{l}\text { Whittaker and Niering 1968; Whittaker and } \\
\text { Niering 1975) }\end{array}$ \\
\hline Abies lasiocarpa & 0.227 & $\begin{array}{l}\text { (Whittaker and Niering 1968; Whittaker and } \\
\text { Niering 1975) }\end{array}$ \\
\hline Abies procera & 0.182 & (Fujimori et al. 1976) \\
\hline Abies sachalinensis & 0.233 & (Satoo 1973) \\
\hline Abies veitchii & 0.294 & (Tadaki et al. 1967) \\
\hline Abies veitchii & 0.208 & (Tadaki et al. 1967) \\
\hline Abies veitchii & 0.192 & (Tadaki et al. 1967) \\
\hline Abies veitchii & 0.196 & (Tadaki et al. 1967) \\
\hline Abies veitchii & 0.345 & (Tadaki et al. 1967) \\
\hline Abies veitchii & 0.185 & (Tadaki et al. 1967) \\
\hline Abies veitchii & 0.213 & (Tadaki et al. 1967) \\
\hline Abies veitchii & 0.313 & (Tadaki et al. 1967) \\
\hline Abies veitchii & 0.233 & (Tadaki et al. 1967) \\
\hline Abies veitchii & 0.286 & (Kimura 1963; Kimura 1969; Kimura et al. 1968) \\
\hline Abies veitchii & 0.161 & (Kimura 1963; Kimura 1969; Kimura et al. 1968) \\
\hline Abies veitchii & 0.175 & (Kimura 1963; Kimura 1969; Kimura et al. 1968) \\
\hline Abies veitchii & 0.161 & (Kimura 1963; Kimura 1969; Kimura et al. 1968) \\
\hline Abies veitchii & 0.139 & (Kimura 1963; Kimura 1969; Kimura et al. 1968) \\
\hline Abies veitchii & 0.204 & (Kimura 1963; Kimura 1969; Kimura et al. 1968) \\
\hline Abies veitchii & 0.185 & (Kimura 1963; Kimura 1969; Kimura et al. 1968) \\
\hline Picea abies & 0.141 & $\begin{array}{l}\text { (Duvigneaud and Kestemont 1977; Kestemont } \\
\text { 1975) }\end{array}$ \\
\hline Picea abies & 0.189 & (Droste zu Hülshoff 1970; Ellenberg 1981a) \\
\hline Picea abies & 0.244 & (Droste zu Hülshoff 1970; Ellenberg 1981a) \\
\hline Picea abies & 0.400 & (Droste zu Hülshoff 1970; Ellenberg 1981a) \\
\hline Picea abies & 0.182 & (Satoo 1971) \\
\hline Picea abies & 0.313 & (Satoo 1971) \\
\hline Picea abies & 0.323 & (Satoo 1971) \\
\hline Picea abies & 0.130 & (Satoo 1971) \\
\hline Picea abies & 0.189 & (Satoo 1971; Yoshimura 1967) \\
\hline Picea abies & 0.182 & $\begin{array}{l}\text { (Nihlgard 1972; Nihlgard and Lindgren 1977; } \\
\text { Nihlgard and Lindgren 1981) }\end{array}$ \\
\hline Picea rubens & 0.088 & (Gordon 1981) \\
\hline Picea rubens & 0.169 & (Gordon 1981) \\
\hline Picea rubens & 0.123 & (Gordon 1981) \\
\hline Picea rubens & 0.125 & (Gordon 1981) \\
\hline Pinus banksiana & 0.286 & (Gordon 1981) \\
\hline Pinus banksiana & 0.303 & (Gordon 1981) \\
\hline
\end{tabular}


Earth Interactions - Volume 4 (2000) - Paper No. 3 - Page 17

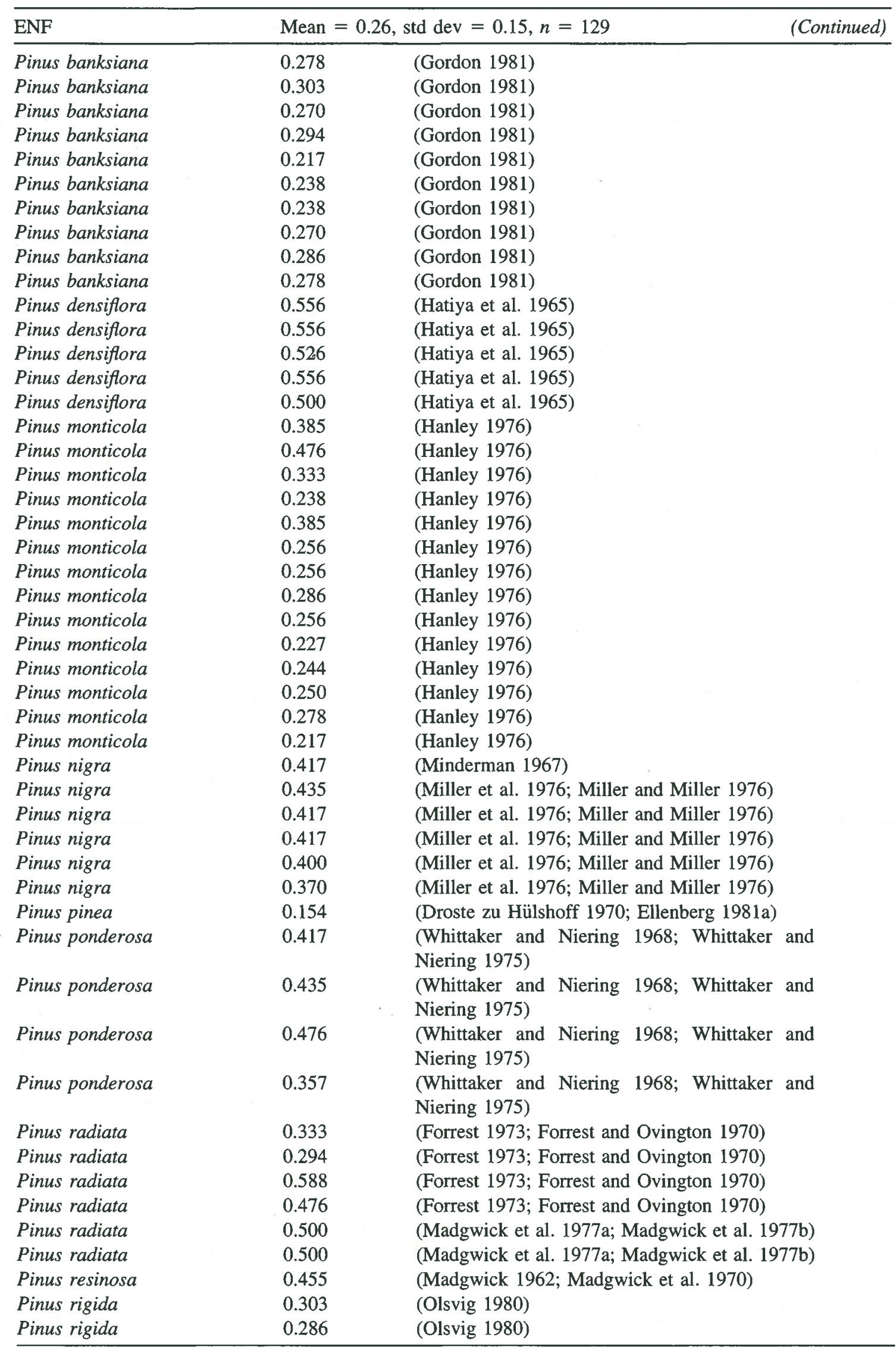




\begin{tabular}{|c|c|c|}
\hline ENF & Mean & std $\operatorname{dev}=0.15, n=129$ \\
\hline Pinus rigida & 0.278 & (Olsvig 1980) \\
\hline Pinus rigida & 0.417 & (Olsvig 1980) \\
\hline Pinus strobus & 0.769 & $\begin{array}{l}\text { (Swank and Schreuder 1973; Swank and Schreu- } \\
\text { der 1974) }\end{array}$ \\
\hline Pinus sylvestris & 0.385 & (Mälkönen 1974) \\
\hline Pinus sylvestris & 0.400 & (Mälkönen 1974) \\
\hline Pinus sylvestris & 0.400 & (Mälkönen 1974) \\
\hline Pinus sylvestris & 0.345 & (Alvera 1973; Alvera 1981) \\
\hline Pinus taeda & 1.00 & (Nemeth 1973a; Nemeth 1973b) \\
\hline Pinus taeda & 0.435 & (Nemeth 1973a; Nemeth 1973b) \\
\hline Pinus taeda & 0.556 & (Nemeth 1973a; Nemeth 1973b) \\
\hline Pinus taeda & 0.435 & (Nemeth 1973a; Nemeth 1973b) \\
\hline Pinus taeda & 0.909 & (Ralston 1973) \\
\hline Pinus taeda & 0.588 & (Wells et al. 1975) \\
\hline Pinus taeda & 0.476 & (Nemeth 1973a; Nemeth 1973b) \\
\hline Pinus virginiana & 0.588 & (Madgwick 1968) \\
\hline Pseudotsuga menziesii & 0.256 & (Turner 1981; Turner and Long 1975) \\
\hline Pseudotsuga menziesii & 0.233 & (Turner 1981; Turner and Long 1975) \\
\hline Pseudotsuga menziesii & 0.213 & (Turner 1981; Turner and Long 1975) \\
\hline Pseudotsuga menziesii & 0.286 & $\begin{array}{l}\text { (Cole et al. 1968; Cole et al. 1981; Dice 1970; } \\
\text { Grier et al. 1974) }\end{array}$ \\
\hline Pseudotsuga menziesii & 0.217 & $\begin{array}{l}\text { (Cole et al. 1968; Cole et al. 1981; Dice 1970; } \\
\text { Grier et al. 1974) }\end{array}$ \\
\hline Pseudotsuga menziesii & 0.200 & (Keyes and Grier 1981) \\
\hline Pseudotsuga menziesii & 0.200 & (Keyes and Grier 1981) \\
\hline Pseudotsuga menziesii & 0.250 & $\begin{array}{l}\text { (Gholz 1982; Gholz et al. 1976; Gholz et al. } \\
\text { 1979) }\end{array}$ \\
\hline Pseudotsuga menziesii & 0.250 & $\begin{array}{l}\text { (Gholz 1982; Gholz et al. 1976; Gholz et al. } \\
\text { 1979) }\end{array}$ \\
\hline Pseudotsuga menziesii & 0.222 & $\begin{array}{l}\text { (Gholz 1982; Gholz et al. 1976; Gholz et al. } \\
\text { 1979) }\end{array}$ \\
\hline Pseudotsuga menziesii & 0.417 & (Turner 1981; Turner and Long 1975) \\
\hline Pseudotsuga menziesii & 0.500 & (Turner 1981; Turner and Long 1975) \\
\hline Pseudotsuga menziesii & 0.323 & (Turner 1981; Turner and Long 1975) \\
\hline Pseudotsuga menziesii & 0.270 & (Turner 1981; Turner and Long 1975) \\
\hline Pseudotsuga menziesii & 0.213 & $\begin{array}{l}\text { (Whittaker and Niering 1968; Whittaker and } \\
\text { Niering 1975) }\end{array}$ \\
\hline Pseudotsuga menziesii & 0.196 & $\begin{array}{l}\text { (Whittaker and Niering 1968; Whittaker and } \\
\text { Niering 1975) }\end{array}$ \\
\hline Pseudotsuga menziesii & 0.313 & $\begin{array}{l}\text { (Gholz 1982; Gholz et al. 1976; Gholz et al. } \\
\text { 1979) }\end{array}$ \\
\hline Tsuga diversifolia & 0.213 & (Kitazawa 1981) \\
\hline Tsuga heterophylla & 0.294 & (Fujimori 1971; Fujimori et al. 1976; Grier 1976) \\
\hline Tsuga heterophylla & 0.370 & (Fujimori 1971; Fujimori et al. 1976; Grier 1976) \\
\hline Tsuga sieboldii & 0.294 & (Ando et al. 1977) \\
\hline DBF & 1.00 & Annual canopy turnover \\
\hline DNF & 1.00 & Annual canopy turnover \\
\hline Grass & 1.00 & Annual canopy turnover \\
\hline Shrub & 0.320 & Set to ENF \\
\hline
\end{tabular}




\section{Earth Interactions - Volume 4 (2000) - Paper No. 3 - Page 19}

\section{A.1.2 Live wood turnover}

We are unaware of any appropriate data with which to parameterize live wood turnover (LWT, $1 \mathrm{yr}^{-1}$ ). Since cambium (conceptually the live wood pool in BIOME-BGC) is replaced on an annual basis, LWT could be set to 1.0. However, since the living and respiring portion of the sapwood [primarily ray parenchyma (Kozlowski and Pallardy 1997)] originates from the cambium, some of the live wood must be retained, and we set LWT to 0.7 for all woody biomes.

\section{A.1.3 Whole plant mortality}

Whole plant mortality (WPM, $1 \mathrm{yr}^{-1}$, including whole-tree death, branch shedding, herbivory, etc.) is the fraction of the above- and below-ground ecosystem carbon pools that dies or is consumed each year. Silvicultural researchers have collected large amounts of data on self-thinning processes and age-density relationships, but because BIOME-BGC requires a proportion of the stand (mass or volume) that dies each year in mature (not developing) stands, these data are not useable in the parameterization. Data in the required form are scarce. The forest value used here $(0.0050)$ is based on a single ongoing large-scale field experiment being conducted by silvicultural researchers and is considered to represent mostly branch and tree mortality (R. E. Keane, USDA Forest Service, 1998, personal communication). Grass WPM $(0.10)$ is meant to represent herbivory, which varies greatly with insect phenology and the presence or absence of grazing activity and can range from 0.06 in steppe (Lavrenko and Karamysheva 1992) to over 0.4 in savanna grasses (Gandar 1982). Our value is thus a low approximation. Shrub WPM (0.020) is set intermediate between the forest and grass biomes on the assumption that while there is a woody component to the biome, it is small enough that herbivory can still consume significant amounts.

\section{A.1.4 Fire mortality}

Fire mortality $\left(1 \mathrm{yr}^{-1}\right)$ is based on approximations from data in Aber and Melillo (Aber and Melillo 1991). Based on their general co-occurrence, we set the DNF FM to the ENF value (0.0050). DBF FM is significantly lower (0.0025). Use of the low end of Aber and Melillo's (Aber and Melillo 1991) prairie fire regime of 0.1 resulted in extremely low simulated grass LAI and we reduced grass FM to 0.05. Data from grass-dominated tropical savannas suggest that FM of 0.05 (20yr interval) is not uncommon (Lacey et al. 1982). To represent reduced fire rate in cold shrublands, we set shrub FM to 0.010 , slightly below the low shrub value in Aber and Melillo (Aber and Melillo 1991).

\section{A.2 Allocation parameters}

Allometric relationships between different plant pools control how photosynthetically produced carbon is allocated throughout the ecosystem. BIOME-BGC considers carbon allocation to major plant pools of roots (fine and coarse), stems, and leaves. The four ratios presented below are used in an algebraic solution to determine the allocation of carbon throughout the ecosystem. As each plant pool 


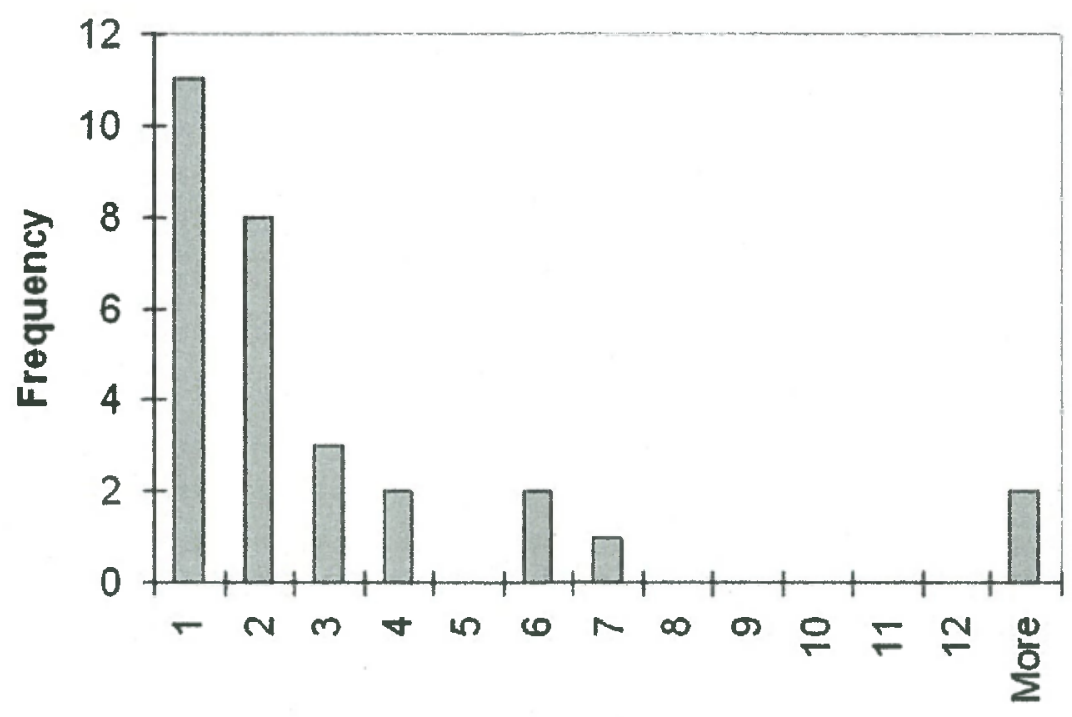

FRC:LC

Figure A1. Frequency histogram of the ratio of new fine root carbon to new leaf carbon.

is associated with a fixed carbon to nitrogen ratio (below), allocation indirectly controls nitrogen demand.

Carbon allocation to seeds (Kaldy and Dunton 1999), fruit (Jonasson et al. 1997), and defensive chemistry (Crone and Jones 1999; Wallin and Raffa 1999) can represent a significant portion of total allocation, but the physiologic, genetic, and pathogenic detail required to accurately model these processes is impractical in a generalized ecosystem model. Site-specific application of BIOME-BGC or other BGC models should consider these processes.

The allocation parameters were, in general, well documented from a wealth of stand inventory data collected from the 1960s-1980s. However, little data were available for the DNF biome. DNF allocation exhibits similarities to both ENF and DBF patterns (Gower and Richards 1990) without complete justification for adopting either strategy as a surrogate for DNF. We arbitrarily chose to set DNF equal to ENF values.

\section{A.2.1 New fine root carbon to new leaf carbon allocation}

In spite of the great difficulty in measuring FRC:LC $\left(\mathrm{kg} \mathrm{C} \mathrm{kg} \mathrm{C}^{-1}\right)$, there was a surprisingly large amount of data available, usually recorded separately as fine root and leaf NPP. The distribution of FRC:LC for ENF, upon which three biome values are based, was highly positively skewed, with the mean value (2.7) almost twice the median (Figure A1). Ratios close to one often produce skewed distributions because of the limited possible range of values less than one versus the unlimited range of values greater than one. Inverting the ratio to LC:FRC, for example, removes the skewness. Selecting the mean value also produced an indefensibly large allocation of carbon to fine roots. Even though nitrogen and water 
were both available, LAI development with FRC:LC at the mean value was severely photosynthetically limited by fine root carbon consumption. With FRC:LC set at the mean, ENF did not grow an LAI above 1.9, even at relatively warm and wet sites two and four (appendix B). Thus, we used the median value. We also checked all other ratio values and found that ENF FRC:LC was the only case with extreme skewness.

\begin{tabular}{|c|c|c|}
\hline ENF & Mediar & oseudo-std dev $=1.5, n=29$ \\
\hline Abies & 12.4 & (Vogt et al. 1982) \\
\hline Abies amabilis & 5.46 & (Grier et al. 1981) \\
\hline Abies amabilis & 12.7 & (Grier et al. 1981) \\
\hline Mixed pine & 0.931 & (Nadelhoffer et al. 1985) \\
\hline Picea & 0.662 & (Nadelhoffer et al. 1985) \\
\hline Pinus contorta & 3.64 & (Comeau and Kimmins 1989) \\
\hline Pinus contorta & 5.50 & (Comeau and Kimmins 1989) \\
\hline Pinus contorta & 2.76 & (Comeau and Kimmins 1989) \\
\hline Pinus contorta & 1.47 & (Comeau and Kimmins 1989) \\
\hline Pinus elliottii & 1.09 & (Gholz et al. 1986) \\
\hline Pinus radiata & 0.463 & (Beets and Pllock 1987) \\
\hline Pinus radiata & 0.347 & (Beets and Pllock 1987) \\
\hline Pinus resinosa & 0.872 & (Nadelhoffer et al. 1985) \\
\hline Pinus strobus & 0.994 & (Nadelhoffer et al. 1985) \\
\hline Pinus sylvestris & 1.03 & (Mälkönen 1974) \\
\hline Pinus sylvestris & 0.921 & (Mälkönen 1974) \\
\hline Pinus sylvestris & 1.17 & (Mälkönen 1974) \\
\hline Pinus sylvestris & 1.37 & (Paavilainen 1980) \\
\hline Pinus sylvestris & 2.99 & (Linder and Axelsson 1982) \\
\hline Pinus sylvestris & 0.523 & (Linder and Axelsson 1982) \\
\hline Pinus taeda & 1.76 & (Kinerson et al. 1977) \\
\hline Pseudotsuga menziesii & 3.66 & (Keyes and Grier 1981) \\
\hline Pseudotsuga menziesii & 0.819 & (Keyes and Grier 1981) \\
\hline Pseudotsuga menziesii & 1.41 & (Vogt et al. 1990) \\
\hline Pseudotsuga menziesii & 0.883 & (Vogt et al. 1990) \\
\hline Pseudotsuga menziesii & 2.41 & (Gower et al. 1992) \\
\hline Pseudotsuga menziesii & 1.00 & (Gower et al. 1992) \\
\hline Pseudotsuga menziesii & 1.43 & (Gower et al. 1992) \\
\hline Pseudotsuga menziesii & 6.85 & (Fogel 1983) \\
\hline DNF & 1.4 & Set to ENF \\
\hline DBF & Mean & $\mathrm{d} \mathrm{dev}=1.2, n=9$ \\
\hline Quercus velutina & 1.59 & (Nadelhoffer et al. 1985) \\
\hline Quercus rubra & 1.39 & (Nadelhoffer et al. 1985) \\
\hline Quercus alba & 1.27 & (Nadelhoffer et al. 1985) \\
\hline Acer saccharum & 1.55 & (Nadelhoffer et al. 1985) \\
\hline Betula & 1.26 & (Nadelhoffer et al. 1985) \\
\hline Fagus & 1.43 & (van Praag et al. 1988) \\
\hline Quercus & 0.673 & (Joslin and Henderson 1987) \\
\hline Fagus & 0.545 & (Ellenberg et al. 1986) \\
\hline Nyssa-Acer & 1.44 & (Symbula and Day 1988) \\
\hline Grass & Mean & $\mathrm{td} \operatorname{dev}=0.54, n=32$ \\
\hline Salt marsh & 1.00 & (Bliss 1977) \\
\hline Wet meadow & 0.338 & (Bliss 1977) \\
\hline
\end{tabular}


Earth Interactions - Volume 4 (2000) - Paper No. 3 - Page 22

\begin{tabular}{lll}
\hline Grass & Mean $=1.0$, std dev $=0.54, n=32$ & (Continued) \\
\hline Herb meadow & 1.31 & (Tieszen et al. 1981, see Lewis personal com- \\
& & munication) \\
Grass-herb meadow & 1.01 & (Østbye and et al. 1975) \\
Dry grassland & 2.19 & (Lewis Smith and Walton 1975) \\
Grass turf & 0.500 & (Collins et al. 1975) \\
Grassland & 2.00 & (Jenkin 1975) \\
Wet grassland & 0.199 & (Lewis Smith and Walton 1975) \\
Dry meadow & 1.02 & (Wielgolaski 1975) \\
Wet meadow & 1.63 & (Wielgolaski 1975) \\
Grass savanna & 1.59 & (Menaut and Cesar 1979) \\
Grass savanna & 1.43 & (Menaut and Cesar 1979) \\
Grass savanna & 1.32 & (Menaut and Cesar 1979) \\
Temperate grassland & 1.07 & (Sims and Coupland 1979) \\
Temperate grassland & 0.643 & (Sims and Coupland 1979) \\
Temperate grassland & 0.405 & (Sims and Coupland 1979) \\
Temperate grassland & 0.960 & (Sims and Coupland 1979) \\
Mixed grass & 0.281 & (Kumar and Joshi 1972) \\
Mixed grass & 0.470 & (Singh and Yadava 1974) \\
Eragrostis & 0.342 & (Singh 1972) \\
Desmostachya & 0.621 & (Singh 1972) \\
Sehima-Heteropogon & 0.488 & (Shankar et al. 1973) \\
Dichanthium & 0.892 & (Misra 1973) \\
Sehima & 0.921 & (Billore 1973) \\
Mixed grass & 1.29 & (Naik 1973) \\
Heteropogon-Apluda-Cymbopo-1.03 & (Jain 1971) \\
gon & & \\
Tropical grassland & 1.78 & (Singh et al. 1979) \\
Tropical grassland & 0.515 & (Singh et al. 1979) \\
Tropical grassland & 1.00 & (Singh et al. 1979) \\
Tropical grassland & 2.03 & (Singh et al. 1979) \\
Shortgrass steppe & 0.988 & (Milchunas and Laurenroth 1992) \\
Konza prairie & 0.744 & (Hayes and Seastedt 1987) \\
Shrub & 1.4 & Set to ENF \\
\hline & & \\
\hline
\end{tabular}

\section{A.2.2 New stem carbon to new leaf carbon allocation}

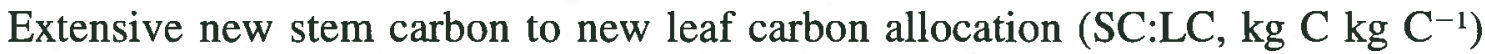
data were available for ENF and DBF and showed identical values. However, preliminary testing showed that with shrub SC:LC set to 2.2 , very large stem carbon accumulation occurred, and we reduced shrub SC:LC to $10 \%$ of the forest values.

\begin{tabular}{|c|c|c|}
\hline \multirow{2}{*}{$\frac{\mathrm{ENF}}{\text { Abies amabilis }}$} & \multicolumn{2}{|c|}{ Mean $=2.2$, std dev $=0.89, n=29$} \\
\hline & 3.58 & $\begin{array}{l}\text { (Gholz et al. 1976; Grier and Milne 1981; Grier } \\
\text { et al. 1981) }\end{array}$ \\
\hline Abies amabilis & 3.37 & $\begin{array}{l}\text { (Gholz et al. 1976; Grier and Milne 1981; Grier } \\
\text { et al. 1981) }\end{array}$ \\
\hline Abies balsamea & 1.02 & (Baskerville 1965; Baskerville 1966) \\
\hline Abies balsamea & 1.14 & (Baskerville 1965; Baskerville 1966) \\
\hline Abies balsamea & 1.13 & (Baskerville 1965; Baskerville 1966) \\
\hline Abies balsamea & 1.16 & (Baskerville 1965; Baskerville 1966) \\
\hline
\end{tabular}




\section{Earth Interactions • Volume 4 (2000) - Paper No. 3 - Page 23}

\begin{tabular}{|c|c|c|}
\hline \multirow{2}{*}{$\frac{\mathrm{ENF}}{\text { Abies balsamea }}$} & \multicolumn{2}{|c|}{ Mean $=2.2$, std dev $=0.89, n=29$} \\
\hline & 1.30 & (Baskerville 1965; Baskerville 1966) \\
\hline Abies balsamea & 1.34 & (Baskerville 1965; Baskerville 1966) \\
\hline Abies concolor & 1.69 & $\begin{array}{l}\text { (Whittaker and Niering 1968; Whittaker and } \\
\text { Niering 1975) }\end{array}$ \\
\hline Abies fraseri & 2.29 & (Whittaker 1966) \\
\hline Abies fraseri & 3.12 & (Whittaker 1966) \\
\hline Abies fraseri & 1.61 & (Whittaker 1966) \\
\hline Abies fraseri & 1.71 & (Whittaker 1966) \\
\hline Abies lasiocarpa & 1.36 & $\begin{array}{l}\text { (Whittaker and Niering 1968; Whittaker and } \\
\text { Niering 1975) }\end{array}$ \\
\hline Abies procera & 3.03 & (Fujimori et al. 1976) \\
\hline Abies sachalinensis & 3.32 & (Satoo 1973) \\
\hline Abies veitchii & 2.56 & (Tadaki et al. 1967) \\
\hline Abies veitchii & 4.17 & (Tadaki et al. 1967) \\
\hline Abies veitchii & 3.03 & (Tadaki et al. 1967) \\
\hline Abies veitchii & 2.61 & (Tadaki et al. 1967) \\
\hline Abies veitchii & 1.64 & (Tadaki et al. 1967) \\
\hline Abies veitchii & 2.50 & (Tadaki et al. 1967) \\
\hline Abies veitchii & 2.42 & (Tadaki et al. 1967) \\
\hline Abies veitchii & 1.34 & (Tadaki et al. 1967) \\
\hline Abies veitchii & 1.55 & (Tadaki et al. 1967) \\
\hline Abies veitchii & 1.64 & (Kimura 1963; Kimura 1969; Kimura et al. 1968) \\
\hline Abies veitchii & 1.62 & (Kimura 1963; Kimura 1969; Kimura et al. 1968) \\
\hline Abies veitchii & 2.36 & (Kimura 1963; Kimura 1969; Kimura et al. 1968) \\
\hline Abies veitchii & 2.37 & (Kimura 1963; Kimura 1969; Kimura et al. 1968) \\
\hline Abies veitchii & 2.95 & (Kimura 1963; Kimura 1969; Kimura et al. 1968) \\
\hline Abies veitchii & 3.20 & (Kimura 1963; Kimura 1969; Kimura et al. 1968) \\
\hline Abies veitchii & 1.06 & (Kimura 1963; Kimura 1969; Kimura et al. 1968) \\
\hline Picea abies & 4.70 & $\begin{array}{l}\text { (Duvigneaud and Kestemont 1977; Kestemont } \\
\text { 1975) }\end{array}$ \\
\hline Picea abies & 1.76 & (Droste zu Hülshoff 1970; Ellenberg 1981a) \\
\hline Picea abies & 1.43 & (Droste zu Hülshoff 1970; Ellenberg 1981a) \\
\hline Picea abies & 1.45 & (Droste zu Hülshoff 1970; Ellenberg 1981a) \\
\hline Picea abies & 2.68 & (Satoo 1971) \\
\hline Picea abies & 1.51 & (Satoo 1971) \\
\hline Picea abies & 1.48 & (Satoo 1971) \\
\hline Picea abies & 2.35 & (Satoo 1971) \\
\hline Picea abies & 2.09 & (Satoo 1971; Yoshimura 1967) \\
\hline Picea abies & 3.42 & $\begin{array}{l}\text { (Nihlgard 1972; Nihlgard and Lindgren } 1977 \text {; } \\
\text { Nihlgard and Lindgren 1981) }\end{array}$ \\
\hline Picea rubens & 2.17 & (Gordon 1981) \\
\hline Picea rubens & 1.24 & (Gordon 1981) \\
\hline Picea rubens & 2.77 & (Gordon 1981) \\
\hline Picea rubens & 2.34 & (Gordon 1981) \\
\hline Pinus banksiana & 2.39 & (Gordon 1981) \\
\hline Pinus banksiana & 2.45 & (Gordon 1981) \\
\hline Pinus banksiana & 2.22 & (Gordon 1981) \\
\hline Pinus banksiana & 2.22 & (Gordon 1981) \\
\hline Pinus banksiana & 2.27 & (Gordon 1981) \\
\hline Pinus banksiana & 2.19 & (Gordon 1981) \\
\hline Pinus banksiana & 2.19 & (Gordon 1981) \\
\hline Pinus banksiana & 2.16 & (Gordon 1981) \\
\hline Pinus banksiana & 1.82 & (Gordon 1981) \\
\hline
\end{tabular}


Earth Interactions - Volume 4 (2000) - Paper No. 3 - Page 24

\begin{tabular}{|c|c|c|c|}
\hline \multirow{2}{*}{$\frac{\text { ENF }}{\text { Pinus banksiana }}$} & \multicolumn{2}{|c|}{ Mean $=2.2$, std dev $=0.89, n=29$} & \multirow[t]{2}{*}{ (Continued) } \\
\hline & 1.77 & (Gordon 1981) & \\
\hline Pinus banksiana & 1.46 & (Gordon 1981) & \\
\hline Pinus banksiana & 1.43 & (Gordon 1981) & \\
\hline Pinus densiflora & 2.50 & (Hatiya et al. 1965) & \\
\hline Pinus densiflora & 2.46 & (Hatiya et al. 1965) & \\
\hline Pinus densiflora & 2.21 & (Hatiya et al. 1965) & \\
\hline Pinus densiflora & 2.11 & (Hatiya et al. 1965) & \\
\hline Pinus densiflora & 3.18 & (Hatiya et al. 1965) & \\
\hline Pinus monticola & 0.613 & (Hanley 1976) & \\
\hline Pinus monticola & 0.596 & (Hanley 1976) & \\
\hline Pinus monticola & 0.932 & (Hanley 1976) & \\
\hline Pinus monticola & 0.934 & (Hanley 1976) & \\
\hline Pinus monticola & 2.26 & (Hanley 1976) & \\
\hline Pinus monticola & 2.55 & (Hanley 1976) & \\
\hline Pinus monticola & 2.24 & (Hanley 1976) & \\
\hline Pinus monticola & 2.11 & (Hanley 1976) & \\
\hline Pinus monticola & 0.704 & (Hanley 1976) & \\
\hline Pinus monticola & 2.35 & (Hanley 1976) & \\
\hline Pinus monticola & 2.03 & (Hanley 1976) & \\
\hline Pinus monticola & 1.90 & (Hanley 1976) & \\
\hline Pinus monticola & 2.33 & (Hanley 1976) & \\
\hline Pinus monticola & 2.46 & (Hanley 1976) & \\
\hline Pinus nigra & 1.53 & (Minderman 1967) & \\
\hline Pinus nigra & 2.09 & (Miller et al. 1976; Miller and Miller 1976) & \\
\hline Pinus nigra & 2.12 & (Miller et al. 1976; Miller and Miller 1976) & \\
\hline Pinus nigra & 2.10 & (Miller et al. 1976; Miller and Miller 1976) & \\
\hline Pinus nigra & 1.98 & (Miller et al. 1976; Miller and Miller 1976) & \\
\hline Pinus nigra & 1.70 & (Miller et al. 1976; Miller and Miller 1976) & \\
\hline Pinus pinea & 1.89 & (Droste zu Hülshoff 1970; Ellenberg 1981a) & \\
\hline Pinus ponderosa & 0.981 & $\begin{array}{l}\text { (Whittaker and Niering 1968; Whittaker ar } \\
\text { Niering 1975) }\end{array}$ & and \\
\hline Pinus ponderosa & 0.983 & $\begin{array}{l}\text { (Whittaker and Niering 1968; Whittaker an } \\
\text { Niering 1975) }\end{array}$ & and \\
\hline Pinus ponderosa & 0.933 & $\begin{array}{l}\text { (Whittaker and Niering 1968; Whittaker an } \\
\text { Niering 1975) }\end{array}$ & \\
\hline Pinus ponderosa & 0.851 & $\begin{array}{l}\text { (Whittaker and Niering 1968; Whittaker at } \\
\text { Niering 1975) }\end{array}$ & and \\
\hline Pinus radiata & 2.29 & (Forrest 1973; Forrest and Ovington 197) & \\
\hline Pinus radiata & 4.03 & (Forrest 1973; Forrest and Ovington 1970) & \\
\hline Pinus radiata & 3.59 & (Forrest 1973; Forrest and Ovington 1970) & \\
\hline Pinus radiata & 3.30 & (Forrest 1973; Forrest and Ovington 1970) & \\
\hline Pinus radiata & 2.08 & (Madgwick et al. 1977a; Madgwick et al. 1977 & 7b) \\
\hline Pinus radiata & 2.28 & (Madgwick et al. 1977a; Madgwick et al. 1977 & 7b) \\
\hline Pinus resinosa & 1.39 & (Madgwick 1962; Madgwick et al. 1970) & \\
\hline Pinus rigida & 1.61 & (Olsvig 1980) & \\
\hline Pinus rigida & 1.51 & (Olsvig 1980) & \\
\hline Pinus rigida & 1.99 & (Olsvig 1980) & \\
\hline Pinus rigida & 1.28 & (Olsvig 1980) & \\
\hline Pinus strobus & 2.73 & $\begin{array}{l}\text { (Swank and Schreuder 1973; Swank and Schre } \\
\text { der 1974) }\end{array}$ & eu- \\
\hline Pinus sylvestris & 1.80 & (Mälkönen 1974) & \\
\hline Pinus sylvestris & 2.07 & (Mälkönen 1974) & \\
\hline Pinus sylvestris & 2.11 & (Mälkönen 1974) & \\
\hline
\end{tabular}




\section{Earth Interactions - Volume 4 (2000) - Paper No. 3 - Page 25}

\begin{tabular}{|c|c|c|}
\hline ENF & \multicolumn{2}{|c|}{ Mean $=2.2$, std dev $=0.89, n=29$} \\
\hline Pinus sylvestris & 2.51 & (Alvera 1973; Alvera 1981) \\
\hline Pinus taeda & 2.05 & (Nemeth 1973a; Nemeth 1973b) \\
\hline Pinus taeda & 1.98 & (Nemeth 1973a; Nemeth 1973b) \\
\hline Pinus taeda & 3.61 & (Nemeth 1973a; Nemeth 1973b) \\
\hline Pinus taeda & 4.80 & (Nemeth 1973a; Nemeth 1973b) \\
\hline Pinus taeda & 1.99 & (Ralston 1973) \\
\hline Pinus taeda & 1.56 & (Wells et al. 197) \\
\hline Pinus taeda & 1.82 & (Nemeth 1973a; Nemeth 1973b) \\
\hline Pinus virginiana & 1.89 & (Madgwick 1968) \\
\hline Pseudotsuga & 1.65 & (Turner 1981; Turner and Long 1975) \\
\hline Pseudotsuga & 1.69 & (Turner 1981; Turner and Long 1975) \\
\hline Pseudotsuga & 1.24 & (Turner 1981; Turner and Long 1975) \\
\hline Pseudotsuga menziesii & 5.32 & $\begin{array}{l}\text { (Cole et al. 1968; Cole et al. 1981; Dice 1970; } \\
\text { Grier et al. 1974) }\end{array}$ \\
\hline Pseudotsuga menziesii & 3.95 & $\begin{array}{l}\text { (Cole et al. 1968; Cole et al. 1981; Dice 1970; } \\
\text { Grier et al. 1974) }\end{array}$ \\
\hline Pseudotsuga menziesii & 2.65 & (Keyes and Grier 1981) \\
\hline Pseudotsuga menziesii & 3.28 & (Keyes and Grier 1981) \\
\hline Pseudotsuga menziesii & 1.07 & $\begin{array}{l}\text { (Gholz 1982; Gholz et al. 1976; Gholz et al. } \\
\text { 1979) }\end{array}$ \\
\hline Pseudotsuga menziesii & 3.54 & $\begin{array}{l}\text { (Gholz 1982; Gholz et al. 1976; Gholz et al. } \\
\text { 1979) }\end{array}$ \\
\hline Pseudotsuga menziesii & 3.18 & (Turner 1981; Turner and Long 1975) \\
\hline Pseudotsuga menziesii & 1.68 & (Turner 1981; Turner and Long 1975) \\
\hline Pseudotsuga menziesii & 2.63 & (Turner 1981; Turner and Long 1975) \\
\hline Pseudotsuga menziesii & 3.17 & (Turner 1981; Turner and Long 1975) \\
\hline Pseudotsuga menziesii & 1.31 & $\begin{array}{l}\text { (Whittaker and Niering 1968; Whittaker and } \\
\text { Niering 1975) }\end{array}$ \\
\hline Pseudotsuga menziesii & 1.72 & $\begin{array}{l}\text { (Whittaker and Niering 1968; Whittaker and } \\
\text { Niering 1975) }\end{array}$ \\
\hline Pseudotsuga menziesii & 1.10 & $\begin{array}{l}\text { (Gholz 1982; Gholz et al. 1976; Gholz et al. } \\
\text { 1979) }\end{array}$ \\
\hline Pseudotsuga menziesii & 1.38 & $\begin{array}{l}\text { (Gholz 1982; Gholz et al. 1976; Gholz et al. } \\
\text { 1979) }\end{array}$ \\
\hline Tsuga diversifolia & 2.23 & (Kitazawa 1981) \\
\hline Tsuga heterophylla & 4.15 & (Fujimori 1971; Fujimori et al. 1976; Grier 1976) \\
\hline Tsuga heterophylla & 3.63 & (Fujimori 1971; Fujimori et al. 1976; Grier 1976) \\
\hline Tsuga sieboldii & 1.45 & (Ando et al. 1977) \\
\hline DNF & 2.2 & Set to ENF \\
\hline DBF & \multicolumn{2}{|c|}{ Mean $=2.2$, std dev $=1.1, n=133$} \\
\hline Acer platanoides & 2.46 & (Hytteborn 1975) \\
\hline Acer saccharum & 2.29 & (Whittaker 1966; Whittaker 1971) \\
\hline Acer saccharum & 2.29 & (Whittaker 1966; Whittaker 1971) \\
\hline Aesculus octandra & 3.03 & (Whittaker 1966; Whittaker 1971) \\
\hline Alnus glutinosa & 1.78 & (Schlesinger 1978) \\
\hline Alnus glutinosa & 3.48 & $\begin{array}{l}\text { (Nihlgard 1972; Nihlgard and Lindgren 1977; } \\
\text { Nihlgard and Lindgren 1981) }\end{array}$ \\
\hline Alnus incana & 1.83 & (Whittaker 1966; Whittaker 1971) \\
\hline Alnus rubra & 3.17 & (van Cleve et al. 1971) \\
\hline Alnus rubra & 1.85 & $\begin{array}{l}\text { (Zavitkovski et al. 1976; Zavitkovski and Stevens } \\
\text { 1972) }\end{array}$ \\
\hline Alnus rubra & 1.96 & $\begin{array}{l}\text { (Zavitkovski et al. 1976; Zavitkovski and Stevens } \\
\text { 1972) }\end{array}$ \\
\hline
\end{tabular}




\begin{tabular}{|c|c|c|}
\hline DBF & Mean $=$ & $\mathrm{d} \mathrm{dev}=1.1, n=133$ \\
\hline Alnus rubra & 2.00 & $\begin{array}{l}\text { (Zavitkovski et al. 1976; Zavitkovski and Stevens } \\
\text { 1972) }\end{array}$ \\
\hline Alnus rubra & 1.67 & $\begin{array}{l}\text { (Zavitkovski et al. 1976; Zavitkovski and Stevens } \\
\text { 1972) }\end{array}$ \\
\hline Alnus rubra & 1.43 & $\begin{array}{l}\text { (Zavitkovski et al. 1976; Zavitkovski and Stevens } \\
\text { 1972) }\end{array}$ \\
\hline Alnus rubra & 1.33 & $\begin{array}{l}\text { (Zavitkovski et al. 1976; Zavitkovski and Stevens } \\
\text { 1972) }\end{array}$ \\
\hline Betula maximowicziana & 0.933 & (Jakus 1981) \\
\hline Betula maximowicziana & 1.82 & (Satoo 1970; Satoo 1974) \\
\hline Betula maximowicziana & 1.79 & (Satoo 1970; Satoo 1974) \\
\hline Betula pubescens & 4.00 & (Holm and Jensen 1981) \\
\hline Betula pubescens & 1.57 & (Auclair and Méteyer 1980) \\
\hline Betula spp. & 0.920 & (Decei 1981; Donita et al. 1981) \\
\hline Betula verrucosa & 2.78 & (Hughes 1970; Hughes 1971) \\
\hline Betula verrucosa & 3.50 & $\begin{array}{l}\text { (Ovington and Madgwick 1959a; Ovington and } \\
\text { Madgwick 1959b) }\end{array}$ \\
\hline Betula verrucosa & 4.25 & $\begin{array}{l}\text { (Ovington and Madgwick 1959a; Ovington and } \\
\text { Madgwick 1959b) }\end{array}$ \\
\hline Betula verrucosa & 4.53 & $\begin{array}{l}\text { (Ovington and Madgwick 1959a; Ovington and } \\
\text { Madgwick 1959b) }\end{array}$ \\
\hline Betula verrucosa & 4.86 & $\begin{array}{l}\text { (Ovington and Madgwick 1959a; Ovington and } \\
\text { Madgwick 1959b) }\end{array}$ \\
\hline Betula verrucosa & 4.57 & $\begin{array}{l}\text { (Ovington and Madgwick 1959a; Ovington and } \\
\text { Madgwick 1959b) }\end{array}$ \\
\hline Betula verrucosa & 4.92 & $\begin{array}{l}\text { (Ovington and Madgwick 1959a; Ovington and } \\
\text { Madgwick 1959b) }\end{array}$ \\
\hline Betula verrucosa & 4.50 & $\begin{array}{l}\text { (Ovington and Madgwick 1959a; Ovington and } \\
\text { Madgwick 1959b) }\end{array}$ \\
\hline Betula verrucosa & 4.20 & $\begin{array}{l}\text { (Ovington and Madgwick 1959a; Ovington and } \\
\text { Madgwick 1959b) }\end{array}$ \\
\hline Betula verrucosa & 4.19 & $\begin{array}{l}\text { (Ovington and Madgwick 1959a; Ovington and } \\
\text { Madgwick 1959b) }\end{array}$ \\
\hline Carpinus betulus & 1.41 & (Mälkönen 1977) \\
\hline Carya spp. & 1.27 & (Harris et al. 1973; Harris and Henderson 1981) \\
\hline Castanea sativa & 1.50 & $\begin{array}{l}\text { (Ford and Newbould 1970; Ford and Newbould } \\
\text { 1971) }\end{array}$ \\
\hline Castanea sativa & 2.57 & $\begin{array}{l}\text { (Ford and Newbould 1970; Ford and Newbould } \\
\text { 1971) }\end{array}$ \\
\hline Castanea sativa & 3.42 & $\begin{array}{l}\text { (Ford and Newbould 1970; Ford and Newbould } \\
\text { 1971) }\end{array}$ \\
\hline Castanea sativa & 2.03 & $\begin{array}{l}\text { (Ford and Newbould 1970; Ford and Newbould } \\
\text { 1971) }\end{array}$ \\
\hline Fagus crenata & 2.15 & (Satoo 1970; Satoo 1974) \\
\hline Fagus crenata & 2.06 & (Tadaki et al. 1969) \\
\hline Fagus crenata & 2.34 & (Tadaki et al. 1969) \\
\hline Fagus crenata & 2.43 & (Tadaki et al. 1969) \\
\hline Fagus crenata & 1.17 & (Kakubari 1977) \\
\hline Fagus crenata & 1.20 & (Kakubari 1977) \\
\hline Fagus crenata & 1.51 & (Kakubari 1977) \\
\hline Fagus crenata & 0.869 & (Kakubari 1977) \\
\hline Fagus crenata & 0.746 & (Kakubari 1977) \\
\hline Fagus crenata & 0.685 & (Kakubari 1977) \\
\hline
\end{tabular}


Earth Interactions - Volume 4 (2000) - Paper No. 3 - Page 27

\begin{tabular}{|c|c|c|}
\hline \multirow{2}{*}{$\frac{\mathrm{DBF}}{\text { Fagus crenata }}$} & \multicolumn{2}{|c|}{ Mean $=2.2$, std dev $=1.1, n=133$} \\
\hline & 0.827 & (Kakubari 1977) \\
\hline Fagus crenata & 0.747 & (Kakubari 1977) \\
\hline Fagus crenata & 1.80 & (Maruyama 1971; Maruyama 1977) \\
\hline Fagus crenata & 1.81 & (Maruyama 1971; Maruyama 1977) \\
\hline Fagus crenata & 2.25 & (Maruyama 1971; Maruyama 1977) \\
\hline Fagus crenata & 2.02 & (Maruyama 1971; Maruyama 1977) \\
\hline Fagus crenata & 1.73 & (Maruyama 1971; Maruyama 1977) \\
\hline Fagus crenata & 2.08 & (Maruyama 1971; Maruyama 1977) \\
\hline Fagus crenata & 1.36 & (Maruyama 1971; Maruyama 1977) \\
\hline Fagus crenata & 1.68 & (Maruyama 1971; Maruyama 1977) \\
\hline Fagus crenata & 1.31 & (Maruyama 1971; Maruyama 1977) \\
\hline Fagus crenata & 3.11 & (Kawahara et al. 1979; Ogino 1977) \\
\hline Fagus grandifolia & 2.37 & (Turner et al. 1976) \\
\hline Fagus grandifolia & 1.58 & $\begin{array}{l}\text { (Bormann et al. 1970; Gosz et al. 1972; Whittaker } \\
\text { et al. 1974) }\end{array}$ \\
\hline Fagus grandifolia & 1.82 & $\begin{array}{l}\text { (Bormann et al. 1970; Gosz et al. 1972; Whittaker } \\
\text { et al. 1974) }\end{array}$ \\
\hline Fagus grandifolia & 1.87 & $\begin{array}{l}\text { (Bormann et al. 1970; Gosz et al. 1972; Whittaker } \\
\text { et al. 1974) }\end{array}$ \\
\hline Fagus grandifolia & 1.10 & (Whittaker 1966; Whittaker 1971; Young 1972) \\
\hline Fagus sylvatica & 3.88 & (Kestemont 1975) \\
\hline Fagus sylvatica & 2.84 & $\begin{array}{l}\text { (Duvigneaud and Kestemont 1977; Kestemont } \\
\text { 1975) }\end{array}$ \\
\hline Fagus sylvatica & 0.533 & (Pollard 1972) \\
\hline Fagus sylvatica & 2.00 & (Auclair and Méteyer 1980) \\
\hline Fagus sylvatica & 1.83 & (Lemée 1978) \\
\hline Fagus sylvatica & 2.80 & (Ellenberg 1971; Ellenberg 1981b) \\
\hline Fagus sylvatica & 2.02 & (Ellenberg 1971; Ellenberg 1981b) \\
\hline Fagus sylvatica & 2.39 & (Hytteborn 1975) \\
\hline Fagus sylvatica & 3.39 & $\begin{array}{l}\text { (Nihlgard 1972; Nihlgard and Lindgren 1977; } \\
\text { Nihlgard and Lindgren 1981) }\end{array}$ \\
\hline Fagus sylvatica & 3.22 & $\begin{array}{l}\text { (Nihlgard 1972; Nihlgard and Lindgren 1977; } \\
\text { Nihlgard and Lindgren 1981) }\end{array}$ \\
\hline Liriodendron tulipifera & 1.22 & (Whittaker 1966; Whittaker 1971; Young 1972) \\
\hline Liriodendron tulipifera & 4.85 & (Whittaker 1966) \\
\hline Liriodendron tulipifera & 0.608 & $\begin{array}{l}\text { (Harris et al. 1977; Reichle et al. 1981; Sollins et } \\
\text { al. 1973) }\end{array}$ \\
\hline Populus davidiana & 1.82 & (Kawahara et al. 1979; Ogino 1977) \\
\hline Populus grandidenta & 0.910 & (Harris et al. 1973; Harris and Henderson 1981) \\
\hline Populus grandidenta & 3.58 & (Koerper and Richardson 1980) \\
\hline Populus grandidenta & 3.13 & (Koerper and Richardson 1980) \\
\hline Populus tremuloides & 2.05 & (Koerper and Richardson 1980) \\
\hline Populus tremuloides & 0.706 & (Bray and Dudkiewicz 1963; Gosz 1980) \\
\hline Populus tremuloides & 1.75 & $\begin{array}{l}\text { (Alban and Niering 1975; Whittaker and Niering } \\
\text { 1975) }\end{array}$ \\
\hline Populus tremuloides & 3.36 & $\begin{array}{l}\text { (Alban and Niering 1975; Whittaker and Niering } \\
\text { 1975) }\end{array}$ \\
\hline Populus tremuloides & 2.45 & (Crow 1978) \\
\hline Populus tremuloides & 1.69 & (Crow 1978) \\
\hline Populus tremuloides & 2.42 & (Kestemont 1971; Kestemont 1975) \\
\hline Populus tremuloides & 2.77 & (Pollard 1972) \\
\hline Populus tremuloides & 2.38 & (Pollard 1972) \\
\hline Quercus & 5.28 & (Whittaker 1963; Whittaker 1966) \\
\hline
\end{tabular}


Earth Interactions - Volume 4 (2000) - Paper No. 3 - Page 28

\begin{tabular}{|c|c|c|}
\hline DBF & Mean & $\mathrm{d} \mathrm{dev}=1.1, n=133$ \\
\hline Quercus alba & 1.03 & (Crow 1978) \\
\hline Quercus alba & 1.36 & (Lawson et al. 1981) \\
\hline Quercus alba & 1.09 & (Lawson et al. 1981) \\
\hline Quercus alba & 0.800 & $\begin{array}{l}\text { (Rochow 1974a; Rochow 1974b; Rochow 1975; } \\
\text { Whittaker 1966) }\end{array}$ \\
\hline Quercus borealis & 3.43 & $\begin{array}{l}\text { (Rochow 1974a; Rochow 1974b; Rochow 1975; } \\
\text { Whittaker 1966) }\end{array}$ \\
\hline Quercus borealis & 1.68 & $\begin{array}{l}\text { (Ovington et al. 1963; Whittaker 1963; Whittaker } \\
\text { 1966) }\end{array}$ \\
\hline Quercus ellipsoidalis & 1.17 & $\begin{array}{l}\text { (Ovington et al. 1963; Whittaker 1963; Whittaker } \\
\text { 1966) }\end{array}$ \\
\hline Quercus oblongifolia & 1.85 & $\begin{array}{l}\text { (Reiners 1972; Reiners and Reiners 1970; Whit- } \\
\text { taker and Niering 1975) }\end{array}$ \\
\hline Quercus pedunculiflora & 0.803 & (Decei 1981; Donita et al. 1981) \\
\hline Quercus petraea & 2.89 & $\begin{array}{l}\text { (Duvigneaud and Kestemont 1977; Kestemont } \\
\text { 1975) }\end{array}$ \\
\hline Quercus petraea & 1.73 & $\begin{array}{l}\text { (Duvigneaud and Froment 1969; Duvigneaud and } \\
\text { Kestemont 1977; Duvigneaud et al. 1971) }\end{array}$ \\
\hline Quercus petraea & 2.70 & (Ellenberg 1971; Ellenberg 1981b) \\
\hline Quercus petraea & 3.00 & (Satoo 1970; Satoo et al. 1956) \\
\hline Quercus prinus & 1.57 & $\begin{array}{l}\text { (Reiners 1972; Reiners and Reiners 1970; Whit- } \\
\text { taker and Niering 1975) }\end{array}$ \\
\hline Quercus prinus & 3.33 & (Whittaker 1963; Whittaker 1966) \\
\hline Quercus prinus & 1.32 & (Harris et al. 1973; Harris and Henderson 1981) \\
\hline Quercus pubescens & 1.90 & (van der Drift 1974; van der Drift 1981) \\
\hline Quercus robur & 2.60 & $\begin{array}{l}\text { (Duvigneaud and Froment 1969; Duvigneaud and } \\
\text { Kestemont 1977; Duvigneaud et al. 1971) }\end{array}$ \\
\hline Quercus robur & 2.11 & $\begin{array}{l}\text { (Duvigneaud and Froment 1969; Duvigneaud and } \\
\text { Kestemont 1977; Duvigneaud et al. 1971) }\end{array}$ \\
\hline Quercus robur & 1.79 & $\begin{array}{l}\text { (Duvigneaud and Froment 1969; Duvigneaud and } \\
\text { Kestemont 1977; Duvigneaud et al. 1971) }\end{array}$ \\
\hline Quercus robur & 2.66 & (Kestemont 1971; Kestemont 1975) \\
\hline Quercus robur & 2.01 & (Hytteborn 1975) \\
\hline Quercus stellata & 1.32 & $\begin{array}{l}\text { (Day and Monk 1977a; Day and Monk 1977b; } \\
\text { Day and Monk 1974) }\end{array}$ \\
\hline Taxodium distichum & 1.65 & (Johnson and Risser 1974) \\
\hline Grass & No wo & ponent \\
\hline Shrub & 0.22 & See text for discussion \\
\hline
\end{tabular}

\section{A.2.3 New live wood carbon fo new total wood carbon allocation}

New live wood carbon to new total wood carbon allocation (LWC:TWC, kg C $\mathrm{kg} \mathrm{C}^{-1}$ ) controls the amount of respiring tissue in new wood and is based on the percentage of living parenchyma cells in sapwood. For shrubs we assumed that all stem carbon is live. Since only the living portion of wood is respiring, LWC: TWC is important for stem respiration predictions.

\begin{tabular}{lll}
\hline ENF & Mean $=0.071$, std dev $=0.014, n=8$ \\
\hline Abies balsamea & 0.0560 & (Panshin et al. 1964) \\
Larix occidentalis & 0.100 & (Panshin et al. 1964) \\
Picea engelmannii & 0.0590 & (Panshin et al. 1964) \\
\hline
\end{tabular}




\begin{tabular}{|c|c|c|c|}
\hline \multirow{2}{*}{$\begin{array}{l}\text { ENF } \\
\text { Pinus taeda }\end{array}$} & \multicolumn{2}{|c|}{ Mean $=0.071$, std dev $=0.014, n=8$} & \multirow[t]{2}{*}{ (Continued) } \\
\hline & 0.0760 & (Panshin et al. 1964) & \\
\hline Pseudotsuga menziesii & 0.0730 & (Panshin et al. 1964) & \\
\hline Sequoia sempervirens & 0.0780 & (Panshin et al. 1964) & \\
\hline Taxodium distichum & 0.0660 & (Panshin et al. 1964) & \\
\hline Tsuga canadensis & 0.0590 & (Panshin et al. 1964) & \\
\hline DNF & 0.071 & Set to ENF & \\
\hline DBF & \multicolumn{2}{|c|}{ Mean $=0.16$, std dev $=0.084, n=8$} & \\
\hline Acer saccharum & 0.179 & (Panshin et al. 1964) & \\
\hline Betula alleghaniensis & 0.107 & (Panshin et al. 1964) & \\
\hline Fagus grandifolia & 0.204 & (Panshin et al. 1964) & \\
\hline Liriodendron tulipifera & 0.142 & (Panshin et al. 1964) & \\
\hline Populus tremuloides & 0.096 & (Panshin et al. 1964) & \\
\hline Quercus alba & 0.279 & (Panshin et al. 1964) & \\
\hline Robinia pseudoacacia & 0.209 & (Panshin et al. 1964) & \\
\hline Tilia americana & 0.00600 & (Panshin et al. 1964) & \\
\hline Grass & \multicolumn{2}{|c|}{ No woody component } & \\
\hline Shrub & 1.0 & See text for discussion & \\
\hline
\end{tabular}

\section{A.2.4 Coarse root carbon to stem carbon allocation}

Coarse root carbon to stem carbon allocation (CRC:SC, $\mathrm{kg} \mathrm{C} \mathrm{kg} \mathrm{C}^{-1}$ ) was well documented and similar between the ENF and DBF biomes. Together with FRC: LC, CRC:SC is important for determining the mass and respiration of above- and below-ground portions of the ecosystem.

\begin{tabular}{lll}
\hline ENF & Mean $=0.29$, std dev $=0.14, n=56$ \\
\hline Abies amabilis & 0.395 & $\begin{array}{l}\text { (Gholz et al. 1979; Grier and Milne 1981; Grier } \\
\text { et al. 1981) }\end{array}$ \\
(Gholz et al. 1979; Grier and Milne 1981; Grier \\
Abies amabilis & 0.202 & $\begin{array}{l}\text { et al. 1981) } \\
\text { (Tadaki et al. 1967) }\end{array}$ \\
Abies veitchii & 0.659 & (Tadaki et al. 1967) \\
Abies veitchii & 0.298 & (Tadaki et al. 1967) \\
Abies veitchii & 0.301 & (Tadaki et al. 1967) \\
Abies veitchii & 0.302 & (Tadaki et al. 1970) \\
Abies veitchii & 0.488 & (Tadaki et al. 1970) \\
Abies veitchii & 0.318 & (Tadaki et al. 1970) \\
Abies veitchii & 0.413 & (Tadaki et al. 1970) \\
Abies veitchii & 0.273 & (Kimura 1963; Kimura 1969; Kimura et al. 1968) \\
Abies veitchii & 0.235 & (Duvigneaud and Kestemont 1977; Kestemont \\
Abies veitchii & 0.266 & 1975) \\
Picea abies & 0.159 & (Droste zu Hülshoff 1970; Ellenberg 1981b) \\
& (Nihlgard 1972; Nihlgard and Lindgren 1977; \\
Picea abies & 0.194 & Nihlgard and Lindgren 1981) \\
Picea abies & 0.230 & (Hatiya et al. 1965) \\
(Hatiya et al. 1965) & (Hatiya et al. 1965) \\
Pinus densiflora & 0.236 & (Hatiya et al. 1965) \\
Pinus densiflora & 0.246 & (Hatiya et al. 1965) \\
Pinus densiflora & 0.240 & \\
Pinus densiflora & 0.238 & \\
Pinus densiflora & 0.259 &
\end{tabular}


Earth Interactions - Volume 4 (2000) - Paper No. 3 - Page 30

\begin{tabular}{|c|c|c|}
\hline ENF & Mean & std dev $=0.14, n=56$ \\
\hline Pinus monticola & 0.211 & (Hanley 1976) \\
\hline Pinus monticola & 0.250 & (Hanley 1976) \\
\hline Pinus monticola & 0.171 & (Hanley 1976) \\
\hline Pinus monticola & 0.367 & (Hanley 1976) \\
\hline Pinus monticola & 0.483 & (Hanley 1976) \\
\hline Pinus monticola & 0.186 & (Hanley 1976) \\
\hline Pinus monticola & 0.200 & (Hanley 1976) \\
\hline Pinus monticola & 0.165 & (Hanley 1976) \\
\hline Pinus monticola & 0.184 & (Hanley 1976) \\
\hline Pinus monticola & 0.213 & (Hanley 1976) \\
\hline Pinus monticola & 0.203 & (Hanley 1976) \\
\hline Pinus monticola & 0.187 & (Hanley 1976) \\
\hline Pinus monticola & 0.173 & (Hanley 1976) \\
\hline Pinus monticola & 0.174 & (Hanley 1976) \\
\hline Pinus nigra & 0.220 & (Miller et al. 1976; Miller and Miller 1976) \\
\hline Pinus nigra & 0.515 & (Miller et al. 1976; Miller and Miller 1976) \\
\hline Pinus nigra & 0.303 & (Miller et al. 1976; Miller and Miller 1976) \\
\hline Pinus nigra & 0.264 & (Miller et al. 1976; Miller and Miller 1976) \\
\hline Pinus nigra & 0.471 & (Miller et al. 1976; Miller and Miller 1976) \\
\hline Pinus pinea & 0.288 & (Droste zu Hülshoff 1970; Ellenberg 1981b) \\
\hline Pinus sylvestris & 0.593 & (Mälkönen 1974) \\
\hline Pinus sylvestris & 0.375 & (Mälkönen 1974) \\
\hline Pinus sylvestris & 0.351 & (Mälkönen 1974) \\
\hline Pinus taeda & 0.221 & (Nemeth 1973a; Nemeth 1973b) \\
\hline Pinus taeda & 0.228 & (Nemeth 1973a; Nemeth 1973b) \\
\hline Pinus taeda & 0.182 & (Nemeth 1973a; Nemeth 1973b) \\
\hline Pinus taeda & 0.181 & (Nemeth 1973a; Nemeth 1973b) \\
\hline Pinus taeda & 0.841 & $\begin{array}{l}\text { (Harris et al. 1977; Kinerson et al. 1977; Ralston } \\
\text { 1973) }\end{array}$ \\
\hline Pinus taeda & 0.250 & (Nemeth 1973a; Nemeth 1973b) \\
\hline Pseudotsuga menziesii & 0.310 & $\begin{array}{l}\text { (Cole et al. 1968; Cole et al. 1981; Dice 1970; } \\
\text { Grier et al. 1974) }\end{array}$ \\
\hline Pseudotsuga menziesii & 0.151 & $\begin{array}{l}\text { (Cole et al. 1968; Cole et al. 1981; Dice 1970; } \\
\text { Grier et al. 1974) }\end{array}$ \\
\hline Pseudotsuga menziesii & 0.472 & (Keyes and Grier 1981) \\
\hline Pseudotsuga menziesii & 0.257 & (Keyes and Grier 1981) \\
\hline Tsuga heterophylla & 0.214 & (Fujimori 1971; Fujimori et al. 1976; Grier 1976) \\
\hline Tsuga heterophylla & 0.248 & (Fujimori 1971; Fujimori et al. 1976; Grier 1976) \\
\hline Tsuga sieboldii & 0.181 & (Ando et al. 1977) \\
\hline DNF & 0.29 & Set to ENF \\
\hline$\underline{\mathrm{DBF}}$ & Mean & std dev $=0.18, n=46$ \\
\hline Aesculus octandra & 0.247 & (Whittaker 1966; Whittaker 1971) \\
\hline Alnus glutinosa & 0.161 & (Kestemont 1975) \\
\hline Alnus rubra & 0.274 & $\begin{array}{l}\text { (Zavitkovski et al. 1976; Zavitkovski and Stevens } \\
\text { 1972) }\end{array}$ \\
\hline Betula pubescens & 0.452 & (Mälkönen 1977) \\
\hline Betula pubescens & 0.127 & (Lemée 1978) \\
\hline Fagus crenata & 0.162 & (Tadaki et al. 1969) \\
\hline Fagus crenata & 0.227 & (Tadaki et al. 1969) \\
\hline Fagus crenata & 0.134 & (Tadaki et al. 1969) \\
\hline Fagus crenata & 0.165 & (Kakubari 1977) \\
\hline Fagus crenata & 0.168 & (Kakubari 1977) \\
\hline
\end{tabular}


Earth Interactions - Volume 4 (2000) - Paper No. 3 - Page 31

\begin{tabular}{|c|c|c|c|}
\hline \multirow{2}{*}{$\frac{\mathrm{DBF}}{\text { Fagus crenata }}$} & \multicolumn{2}{|c|}{ Mean $=0.22$, std dev $=0.18, n=46$} & \multirow[t]{2}{*}{ (Continued) } \\
\hline & 0.178 & (Kakubari 1977) & \\
\hline Fagus crenata & 0.142 & (Kakubari 1977) & \\
\hline Fagus crenata & 0.126 & (Kakubari 1977) & \\
\hline Fagus crenata & 0.160 & (Kakubari 1977) & \\
\hline Fagus crenata & 0.144 & (Kakubari 1977) & \\
\hline Fagus crenata & 0.134 & (Kakubari 1977) & \\
\hline Fagus crenata & 0.311 & (Maruyama 1971; Maruyama 1977) & \\
\hline Fagus crenata & 0.310 & (Maruyama 1971; Maruyama 1977) & \\
\hline Fagus crenata & 0.288 & (Maruyama 1971; Maruyama 1977) & \\
\hline Fagus crenata & 0.299 & (Maruyama 1971; Maruyama 1977) & \\
\hline Fagus crenata & 0.316 & (Maruyama 1971; Maruyama 1977) & \\
\hline Fagus crenata & 0.297 & (Maruyama 1971; Maruyama 1977) & \\
\hline Fagus crenata & 0.336 & (Maruyama 1971; Maruyama 1977) & \\
\hline Fagus crenata & 0.319 & (Maruyama 1971; Maruyama 1977) & \\
\hline Fagus crenata & 0.354 & (Maruyama 1971; Maruyama 1977) & \\
\hline Fagus crenata & 0.231 & (Kawahara et al. 1979; Ogino 1977) & \\
\hline Fagus grandifolia & 0.310 & $\begin{array}{l}\text { (Bormann et al. 1970; Gosz et al. 1972; Whittaker } \\
\text { et al. 1974) }\end{array}$ & \\
\hline Fagus grandifolia & 0.315 & $\begin{array}{l}\text { (Bormann et al. 1970; Gosz et al. 1972; Whittaker } \\
\text { et al. 1974) }\end{array}$ & \\
\hline Fagus grandifolia & 0.319 & $\begin{array}{l}\text { (Bormann et al. 1970; Gosz et al. 1972; Whittaker } \\
\text { et al. 1974) }\end{array}$ & \\
\hline Fagus sylvatica & 0.161 & $\begin{array}{l}\text { (Duvigneaud and Kestemont 1977; Kestemont } \\
\text { 1975) }\end{array}$ & \\
\hline Fagus sylvatica & 0.216 & $\begin{array}{l}\text { (Duvigneaud and Kestemont 1977; Kestemont } \\
\text { 1975) }\end{array}$ & \\
\hline Fagus sylvatica & 0.135 & (Ellenberg 1971; Ellenberg 1981b) & \\
\hline Fagus sylvatica & 0.090 & (Ellenberg 1971; Ellenberg 1981b) & \\
\hline Fagus sylvatica & 0.077 & (Ellenberg 1971; Ellenberg 1981b) & \\
\hline Fagus sylvatica & 0.197 & (Nihlgard 1972; Nihlgard and Lindgren 1977) & \\
\hline Fagus sylvatica & 0.174 & (Nihlgard 1972; Nihlgard and Lindgren 1977) & \\
\hline Fagus sylvatica & 0.181 & (Nihlgard 1972; Nihlgard and Lindgren 1977) & \\
\hline Liriodendron tulipifera & 0.563 & (Harris et al. 1973; Harris and Henderson 1981) & \\
\hline Populus tremuloides & 0.152 & (Pastor and Bockheim 1981) & \\
\hline Quercus petraea & 0.185 & $\begin{array}{l}\text { (Duvigneaud and Froment 1969; Duvigneaud and } \\
\text { Kestemont 1977; Duvigneaud et al. 1971) }\end{array}$ & \\
\hline Quercus petraea & 0.101 & $\begin{array}{l}\text { (Duvigneaud and Froment 1969; Duvigneaud and } \\
\text { Kestemont 1977; Duvigneaud et al. 1971) }\end{array}$ & \\
\hline Quercus petraea & 0.264 & (van der Drift 1974; van der Drift 1981) & \\
\hline Quercus robur & 0.096 & $\begin{array}{l}\text { (Duvigneaud and Froment 1969; Duvigneaud and } \\
\text { Kestemont 1977; Duvigneaud et al. 1971) }\end{array}$ & \\
\hline Quercus robur & 0.187 & $\begin{array}{l}\text { (Duvigneaud and Froment 1969; Duvigneaud and } \\
\text { Kestemont 1977; Duvigneaud et al. 1971) }\end{array}$ & \\
\hline Quercus robur & 0.157 & (Kestemont 1971; Kestemont 1975) & \\
\hline Quercus robur & 0.195 & (Kestemont 1971; Kestemont 1975) & \\
\hline Grass & $\begin{array}{l}\text { No wo } \\
\text { ponent }\end{array}$ & & \\
\hline Shrub & 0.29 & Set to ENF & \\
\hline
\end{tabular}

\section{A.3 Carbon to nitrogen parameters}

The ratio of carbon to nitrogen $(\mathrm{C}: \mathrm{N})$ is used to characterize the nutrient concentration of leaf, litter, fine root, live wood, and dead wood pools. Usually measured 
as milligrams of nitrogen per gram of dry weight or percent nitrogen, $C: N$ is commonly measured and exerts strong control over plant nitrogen demand, decomposition, and respiration.

\section{A.3.1 Leaf carbon to nitrogen ratio}

Leaf carbon to nitrogen ratio $\left(\mathrm{C}: \mathrm{N}_{\text {leaf }}, \mathrm{kg} \mathrm{C} \mathrm{kg} \mathrm{N}^{-1}\right.$ ) determines three important factors: the nitrogen required to construct leaves (thus LAI), the amount of nitrogen available for investment in photosynthetic machinery (also controlled by PLNR, see below), and leaf respiration rates.

\begin{tabular}{|c|c|c|}
\hline \multirow{2}{*}{$\frac{\text { ENF }}{\text { Juniperus virginia }}$} & \multicolumn{2}{|c|}{ Mean $=42$, std dev $=11, n=25$} \\
\hline & 30.5 & (Reich et al. 1995a) \\
\hline Picea abies & 58.8 & (Berg 1988) \\
\hline Picea abies & 28.1 & (Reich et al. 1995a) \\
\hline Picea glauca & 40.3 & (Reich et al. 1995a) \\
\hline Picea mariana & 41.3 & (Reich et al. 1995a) \\
\hline Pinus albicaulis & 45.4 & (Gower and Richards 1990) \\
\hline Pinus banksiana & 40.3 & (Reich et al. 1995a) \\
\hline Pinus contorta & 51.0 & (Hunt et al. 1988) \\
\hline Pinus contorta & 70.0 & (Fahey et al. 1985) \\
\hline Pinus contorta & 47.6 & (Berg and Ekhbom 1991) \\
\hline Pinus contorta & 35.7 & (Gower et al. 1987) \\
\hline Pinus contorta & 41.6 & (Gower and Richards 1990) \\
\hline Pinus resinosa & 37.0 & (Reich et al. 1995a) \\
\hline Pinus resinosa & 50.0 & (Reich et al. 1995a) \\
\hline Pinus strobus & 29.4 & (Reich et al. 1995a) \\
\hline Pinus strobus & 22.8 & (Reich et al. 1995a) \\
\hline Pinus sylvestris & 33.1 & (Berg 1988) \\
\hline Pinus sylvestris & 36.0 & (Reich et al. 1995a) \\
\hline Pinus sylvestris & 33.1 & (Berg and Ekhbom 1991) \\
\hline Pinus taeda & 42.0 & (Naidu et al. 1993) \\
\hline Pseudotsuga menziesii & 50.0 & (Brix 1981) \\
\hline Pseudotsuga menziesii & 40.0 & (Mitchell and Hinckley 1993) \\
\hline Thuja occidentalis & 58.1 & (Reich et al. 1995a) \\
\hline Thuja occidentalis & 39.1 & (Reich et al. 1995a) \\
\hline Tsuga mertensiana & 41.6 & (Gower and Richards 1990) \\
\hline DNF & \multicolumn{2}{|c|}{ Mean $=27$, std dev $=5.6, n=30$} \\
\hline Larix decidua & 26.0 & (Kloeppel et al. 1998) \\
\hline Larix decidua & 27.8 & (Kloeppel et al. 1998) \\
\hline Larix decidua & 33.6 & (Kloeppel et al. 1998) \\
\hline Larix decidua & 26.3 & (Kloeppel et al. 1998) \\
\hline Larix decidua & 29.8 & (Kloeppel et al. 1998) \\
\hline Larix decidua & 23.7 & (Kloeppel et al. 1998) \\
\hline Larix decidua & 18.9 & (Kloeppel et al. 1998) \\
\hline Larix decidua & 20.0 & (Matyssek and Schulze 1987) \\
\hline Larix eurolepsis & 16.7 & (Matyssek and Schulze 1987) \\
\hline Larix gmelinii & 28.1 & (Kloeppel et al. 1998) \\
\hline Larix laricina & 30.3 & (Kloeppel et al. 1998) \\
\hline Larix laricina & 33.8 & (Kloeppel et al. 1998) \\
\hline Larix laricina & 20.9 & (Kloeppel et al. 1998) \\
\hline Larix laricina & 37.0 & (Kloeppel et al. 1998) \\
\hline Larix leptolepsis & 20.8 & (Matyssek and Schulze 1987) \\
\hline
\end{tabular}


Earth Interactions - Volume 4 (2000) - Paper No. 3 - Page 33

\begin{tabular}{|c|c|c|c|}
\hline \multirow{2}{*}{$\frac{\mathrm{DNF}}{\text { Larix lyallii }}$} & \multicolumn{2}{|c|}{ Mean $=27$, std dev $=5.6, n=30$} & \multirow[t]{2}{*}{ (Continued) } \\
\hline & 22.1 & (Kloeppel et al. 1998) & \\
\hline Larix lyallii & 23.8 & (Gower and Richards 1990) & \\
\hline Larix lyallii & 27.8 & (Richards 1981) & \\
\hline Larix occidentalis & 24.4 & (Kloeppel et al. 1998) & \\
\hline Larix occidentalis & 25.3 & (Kloeppel et al. 1998) & \\
\hline Larix occidentalis & 34.7 & (Kloeppel et al. 1998) & \\
\hline Larix occidentalis & 35.2 & (Kloeppel et al. 1998) & \\
\hline Larix occidentalis & 32.3 & (Kloeppel et al. 1998) & \\
\hline Larix occidentalis & 31.3 & (Kloeppel et al. 1998) & \\
\hline Larix occidentalis & 25.0 & (Gower 1987) & \\
\hline Larix occidentalis & 29.4 & (Gower and Richards 1990) & \\
\hline Larix olgenisis & 32.7 & (Kloeppel et al. 1998) & \\
\hline Larix siberica & 20.2 & (Kloeppel et al. 1998) & \\
\hline Larix siberica & 22.3 & (Kloeppel et al. 1998) & \\
\hline Larix siberica & 18.6 & (Kloeppel et al. 1998) & \\
\hline DBF & \multicolumn{2}{|c|}{ Mean $=25$, std dev $=5.4, n=43$} & \\
\hline Acer rubrum & 23.8 & (Reich et al. 1995a) & \\
\hline Acer rubrum & 25.6 & (Reich et al. 1995a) & \\
\hline Acer saccharum & 25.6 & (Reich et al. 1995a) & \\
\hline Acer saccharum & 28.6 & (Reich et al. 1995a) & \\
\hline Acer saccharum & 32.5 & (Ellsworth and Reich 1992a) & \\
\hline Acer saccharum & 23.5 & (Ellsworth and Reich 1992a) & \\
\hline Acer saccharum & 25.8 & (Ellsworth and Reich 1992a) & \\
\hline Acer saccharum & 31.1 & (Jose and Gillespie 1996) & \\
\hline Alnus glutinosa & 18.5 & (Dawson and Funk 1981) & \\
\hline Alnus incana & 16.3 & (Berg and Ekhbom 1991) & \\
\hline Betula nigra & 21.9 & (Reich et al. 1995a) & \\
\hline Betula papyrifera & 28.7 & (Berg and Ekhbom 1991) & \\
\hline Betula pumila & 33.1 & (Reich et al. 1995a) & \\
\hline Carya glabra & 33.1 & (Jose and Gillespie 1996) & \\
\hline Carya ovata & 25.2 & (Reich et al. 1995a) & \\
\hline Catalpa speciosa & 27.0 & (Reich et al. 1995a) & \\
\hline Celtis occidentalis & 20.9 & (Reich et al. 1995a) & \\
\hline Cornus florida & 35.7 & (Reich et al. 1995a) & \\
\hline Fagus grandifolia & 26.9 & (Jose and Gillespie 1996) & \\
\hline Fraxinus americana & 23.5 & (Reich et al. 1995a) & \\
\hline Fraxinus americana & 23.5 & (Reich et al. 1995a) & \\
\hline Mlex verticillata & 32.3 & (Reich et al. 1995a) & \\
\hline Juglans nigra & 16.9 & (Reich et al. 1995a) & \\
\hline Liriodendron tulipifera & $\begin{array}{l}31.4 \\
26.9\end{array}$ & $\begin{array}{l}\text { (Jose and Gillespie 1996) } \\
\text { (Reich et al. 1995a) }\end{array}$ & \\
\hline Morus rubra & 21.6 & (Reich et al. 1995a) & \\
\hline Populus deltoides & 21.2 & (Reich et al. 1995a) & \\
\hline Populus tremuloides & 22.6 & (Reich et al. 1995a) & \\
\hline Prunus serotina & 24.2 & (Reich et al. 1995a) & \\
\hline Prunus serotina & 18.9 & (Reich et al. 1995a) & \\
\hline Quercus alba & 27.2 & (Jose and Gillespie 1996) & \\
\hline Quercus ellipsoidalis & 23.8 & (Reich et al. 1995a) & \\
\hline Quercus macrocarpa & 21.3 & (Reich et al. 1995a) & \\
\hline Quercus prinus & 35.0 & (Jose and Gillespie 1996) & \\
\hline Quercus rubra & 16.8 & (Reich et al. 1995a) & \\
\hline Quercus nubra & 23.7 & (Reich et al. 1995a) & \\
\hline
\end{tabular}


Earth Interactions - Volume 4 (2000) - Paper No. 3 - Page 34

\begin{tabular}{|c|c|c|c|}
\hline \multirow{2}{*}{$\frac{\mathrm{DBF}}{\text { Quercus rubra }}$} & \multicolumn{2}{|c|}{ Mean $=25$, std dev $=5.4, n=43$} & \multirow{8}{*}{ (Continued) } \\
\hline & 33.1 & (Jose and Gillespie 1996) & \\
\hline Quercus velutina & 34.0 & (Jose and Gillespie 1996) & \\
\hline Rhamnus cathartica & 21.6 & (Reich et al. 1995a) & \\
\hline Rubus alleghaniensis & 16.5 & (Reich et al. 1995a) & \\
\hline Salix dasyclados & 18.7 & (Kull et al. 1998) & \\
\hline Salix viminalis & 20.6 & (Kull et al. 1998) & \\
\hline Ulmus americana & 25.9 & (Reich et al. 1995a) & \\
\hline Grass & \multicolumn{3}{|c|}{ Mean $=25$, std dev $=8.6, n=47$} \\
\hline Aegilops ovata & 17.7 & (Garnier et al. 1997) & \\
\hline Agropyron smithii & 28.1 & (Hunt et al. 1988) & \\
\hline Agropyron sp. & 14.3 & (Garnier et al. 1997) & \\
\hline Andropogon gerardii & 32.9 & (Knapp 1985) & \\
\hline Andropogon gerardii & 58.8 & (Knapp 1985) & \\
\hline Avena barbata & 18.9 & (Garnier et al. 1997) & \\
\hline Avenula bromoides & 24.8 & (Garnier et al. 1997) & \\
\hline Brachypodium distachyon & 29.0 & (Garnier et al. 1997) & \\
\hline Brachypodium phoenicoides & 30.8 & (Garnier et al. 1997) & \\
\hline Brachypodium phoenicoides & 32.5 & (Garnier et al. 1997) & \\
\hline Brachypodium retusum & 24.6 & (Garnier et al. 1997) & \\
\hline Brachypodium retusum & 27.9 & (Garnier et al. 1997) & \\
\hline Bromus erectus & 27.3 & (Garnier et al. 1997) & \\
\hline Bromus erectus & 23.2 & (Garnier et al. 1997) & \\
\hline Bromus erectus & 23.8 & (Garnier et al. 1997) & \\
\hline Bromus hordeadeus & 18.8 & (Garnier et al. 1997) & \\
\hline Bromus lanceolatus & 26.7 & (Garnier et al. 1997) & \\
\hline Bromus madritensis & 23.6 & (Garnier et al. 1997) & \\
\hline Bromus madritensis & 23.0 & (Garnier et al. 1997) & \\
\hline Dactylis glomerata & 23.3 & (Garnier et al. 1997) & \\
\hline Desmazeria rigida & 19.6 & (Garnier et al. 1997) & \\
\hline Dichanthium ischaemum & 24.8 & (Garnier et al. 1997) & \\
\hline Dry alluvial meadow & 30.5 & (Titlyanova and Bazilevich 1979) & \\
\hline Halophytic meadow-steppe & 36.8 & (Titlyanova and Bazilevich 1979) & \\
\hline Hordeum murinum & 16.4 & (Garnier et al. 1997) & \\
\hline Hyparrhenia rufa & 16.4 & (Baruch et al. 1985) & \\
\hline Lolium rigidum & 20.2 & (Garnier et al. 1997) & \\
\hline Matador, Canada & 27.9 & (Coupland and van Dyne 1979) & \\
\hline Meadow-steppe & 22.7 & (Titlyanova and Bazilevich 1979) & \\
\hline Meadow-steppe & 26.9 & (Titlyanova and Bazilevich 1979) & \\
\hline Melica ciliata & 18.7 & (Garnier et al. 1997) & \\
\hline Melica ciliata & 18.2 & (Garnier et al. 1997) & \\
\hline Melinis minutiflora & 14.6 & (Baruch et al. 1985) & \\
\hline Mesohalophytic meadow & 23.3 & (Titlyanova and Bazilevich 1979) & \\
\hline Mesophytic alluvial meadow & 45.5 & (Titlyanova and Bazilevich 1979) & \\
\hline Mesophytic alluvial meadow & 25.5 & (Titlyanova and Bazilevich 1979) & \\
\hline Mesophytic meadow & 23.6 & (Titlyanova and Bazilevich 1979) & \\
\hline Mesophytic meadow & 21.2 & (Titlyanova and Bazilevich 1979) & \\
\hline Panicum virgatum & 38.5 & (Knapp 1985) & \\
\hline Panicum virgatum & 45.0 & (Knapp 1985) & \\
\hline Phleum pratense & 18.7 & (Garnier et al. 1997) & \\
\hline Solling Plateau, Germany & 17.6 & (Titlyanova and Bazilevich 1979) & \\
\hline Ssp. Hispanica & 17.1 & (Garnier et al. 1997) & \\
\hline Steppe meadow & 19.3 & (Titlyanova and Bazilevich 1979) & \\
\hline
\end{tabular}




\begin{tabular}{llll}
\hline Grass & \multicolumn{2}{l}{ Mean $=25$, std dev $=8.6, n=47$} & (Continued) \\
\hline Steppe meadow & 21.9 & (Titlyanova and Bazilevich 1979) \\
Temperate grassland & 27.9 & (Coupland and van Dyne 1979) \\
Vulpia ciliata & 24.0 & (Garnier et al. 1997) \\
Shrub & \multicolumn{1}{l}{ Mean $=35$, std dev $=12, n=9$} \\
\hline Arbutus menziesii & 53.3 & (Field et al. 1983) \\
Heteromeles arbutifolia & 56.7 & (Field et al. 1983) \\
Ledum palustre & 28.5 & (Kudo 1995) \\
Ledum palustre & 30.5 & (Kudo 1995) \\
Ledum palustre & 33.3 & (Kudo 1995) \\
Prosopis glandulosa & 17.0 & (Gausman et al. 1979) \\
Prunus ilicifolia & 32.5 & (Field et al. 1983) \\
Rhamnus californica & 32.8 & (Field et al. 1983) \\
Umbellularia californica & 32.2 & (Field et al. 1983) \\
\hline
\end{tabular}

\section{A.3.2 Litter carbon to nitrogen ratio}

Litter carbon to nitrogen ratio ( $\left.\mathrm{C}: \mathrm{N}_{\text {lit }}, \mathrm{kg} \mathrm{C} \mathrm{kg} \mathrm{N}^{-1}\right)$, reflecting the leaf nitrogen content after retranslocation, is based on data from a wide number of species. Nitrogen retranslocation is $55 \%$ for ENF, $77 \%$ for DNF [calculated from C: $\mathrm{N}_{\text {leaf }}$ and the mean larch retranslocation rate in Gower and Richards (Gower and Richard 1990)], $55 \%$ for DBF, $45 \%$ for grass, and $53 \%$ for shrubs.

\begin{tabular}{lll}
\hline ENF & \multicolumn{2}{l}{ Mean $=93$, std dev $=28, n=43$} \\
\hline Abies amabilis & 110 & (Edmonds 1980) \\
Abies amabilis & 110 & (Ross and Tate 1993) \\
Abies balsamea & 84.7 & (Fyles and McGill 1987) \\
Abies concolor & 68.5 & (Stohlgren 1988) \\
Abies concolor & 69.4 & (Stohlgren 1988) \\
Abies lasiocarpa & 87.3 & (Stump and Binkley 1993) \\
Abies lasiocarpa & 102 & (Taylor et al. 1991) \\
Calocedrus decurrens & 79.4 & (Stohlgren 1988) \\
Picea abies & 116 & (Berg and McClaugherty 1989) \\
Picea abies & 50.5 & (Gower and Son 1992) \\
Picea engelmannii & 93.8 & (Stump and Binkley 1993) \\
Picea engelmannii & 87.7 & (Taylor et al. 1991) \\
Picea glauca & 117 & (Fyles and McGill 1987) \\
Pinus banksiana & 103 & (Fyles and McGill 1987) \\
Pinus contorta & 134 & (Berg and McClaugherty 1989) \\
Pinus contorta & 111 & (Stump and Binkley 1993) \\
Pinus contorta & 135 & (Fahey et al. 1985) \\
Pinus contorta & 128 & (Berg and Ekhbom 1991) \\
Pinus contorta & 49.0 & (Taylor et al. 1991) \\
Pinus elliottii & 143 & (Gholz et al. 1985) \\
Pinus lambertiana & 75.8 & (Stohlgren 1988) \\
Pinus lambertiana & 69.4 & (Stohlgren 1988) \\
Pinus ponderosa & 89.3 & (Hart et al. 1992) \\
Pinus ponderosa & 64.9 & (Hart et al. 1992) \\
Pinus resinosa & 69.4 & (Gower and Son 1992) \\
Pinus resinosa & 116 & (Aber et al. 1990) \\
Pinus resinosa & 90.9 & (Pastor et al. 1984) \\
Pinus strobus & 61.0 & (Gower and Son 1992) \\
\hline & &
\end{tabular}




\section{Earth Interactions - Volume 4 (2000) - Paper No. 3 - Page 36}

\begin{tabular}{|c|c|c|c|}
\hline \multirow{2}{*}{$\frac{\text { ENF }}{\text { Pinus strobus }}$} & \multicolumn{2}{|c|}{ Mean $=93$, std dev $=28, n=43$} & \multirow[t]{2}{*}{ (Continued) } \\
\hline & 114 & (Aber et al. 1990) & \\
\hline Pinus strobus & 104 & (Pastor et al. 1984) & \\
\hline Pinus sylvestris & 132 & (Berg et al. 1984) & \\
\hline Pinus sylvestris & 120 & (Berg and McClaugherty 1989) & \\
\hline Pinus sylvestris & 104 & (Berg and Ekhbom 1991) & \\
\hline Pinus sylvestris & 132 & (Staaf and Berg 1982) & \\
\hline Pseudotsuga menziesii & 100 & (Aber and Melillo 1982) & \\
\hline Pseudotsuga menziesii & 49.8 & (Edmonds 1980) & \\
\hline Pseudotsuga menziesii & 61.0 & (Aber and Melillo 1980) & \\
\hline Sequoiadendron gigant. & 96.2 & (Stohlgren 1988) & \\
\hline Tsuga heterophylla & 83.6 & (Edmonds 1980) & \\
\hline Tsuga heterophylla & 60.2 & (Aber et al. 1990) & \\
\hline Tsuga heterophylla & 51.0 & (Pastor et al. 1984) & \\
\hline DNF & \multicolumn{3}{|c|}{ Mean $=120$, std dev $=24, n=30$} \\
\hline Larix decidua & 113 & (Kloeppel et al. 1998) & \\
\hline Larix decidua & 121 & (Kloeppel et al. 1998) & \\
\hline Larix decidua & 146 & (Kloeppel et al. 1998) & \\
\hline Larix decidua & 114 & (Kloeppel et al. 1998) & \\
\hline Larix decidua & 129 & (Kloeppel et al. 1998) & \\
\hline Larix decidua & 103 & (Kloeppel et al. 1998) & \\
\hline Larix decidua & 82.0 & (Kloeppel et al. 1998) & \\
\hline Larix decidua & 87.0 & (Matyssek and Schulze 1987) & \\
\hline Larix eurolepsis & 73.9 & (Matyssek and Schulze 1987) & \\
\hline Larix gmelinii & 122 & (Kloeppel et al. 1998) & \\
\hline Larix laricina & 132 & (Kloeppel et al. 1998) & \\
\hline Larix laricina & 147 & (Kloeppel et al. 1998) & \\
\hline Larix laricina & 91.0 & (Kloeppel et al. 1998) & \\
\hline Larix laricina & 161 & (Kloeppel et al. 1998) & \\
\hline Larix leptolepsis & 91.3 & (Matyssek and Schulze 1987) & \\
\hline Larix lyallii & 96.2 & (Kloeppel et al. 1998) & \\
\hline Larix lyallii & 104 & (Gower and Richards 1990) & \\
\hline Larix lyallii & 122 & (Richards 1981) & \\
\hline Larix occidentalis & 106 & (Kloeppel et al. 1998) & \\
\hline Larix occidentalis & 110 & (Kloeppel et al. 1998) & \\
\hline Larix occidentalis & 151 & (Kloeppel et al. 1998) & \\
\hline Larix occidentalis & 153 & (Kloeppel et al. 1998) & \\
\hline Larix occidentalis & 140 & (Kloeppel et al. 1998) & \\
\hline Larix occidentalis & 136 & (Kloeppel et al. 1998) & \\
\hline Larix occidentalis & 109 & (Gower 1987) & \\
\hline Larix occidentalis & 126 & (Gower and Richards 1990) & \\
\hline Larix olgenisis & 142 & (Kloeppel et al. 1998) & \\
\hline Larix siberica & 88.0 & (Kloeppel et al. 1998) & \\
\hline Larix siberica & 97.0 & (Kloeppel et al. 1998) & \\
\hline Larix siberica & 80.8 & (Kloeppel et al. 1998) & \\
\hline $\mathrm{DBF}$ & \multicolumn{3}{|c|}{ Mean $=55$, std dev $=16, n=76$} \\
\hline Acer & 49.5 & (Aber and Melillo 1980) & \\
\hline Acer pseudoplatanus & 19.8 & (Bocock 1964) & \\
\hline Acer rubrum & 71.4 & (Aber and Melillo 1982) & \\
\hline Acer rubrum & 75.8 & (Aber et al. 1990) & \\
\hline Acer rubrum & 73.5 & (Aber et al. 1990) & \\
\hline Acer rubrum & 71.4 & (Melillo et al. 1982) & \\
\hline Acer saccharum & 87.8 & (Gosz et al. 1973) & \\
\hline
\end{tabular}




\section{Earth Interactions - Volume 4 (2000) - Paper No. 3 - Page 37}

\begin{tabular}{|c|c|c|c|}
\hline \multirow{2}{*}{$\frac{\mathrm{DBF}}{\text { Acer saccharum }}$} & \multicolumn{2}{|c|}{ Mean $=55$, std dev $=16, n=76$} & \multirow[t]{2}{*}{ (Continued) } \\
\hline & 83.3 & (Aber and Melillo 1982) & \\
\hline Acer saccharum & 60.2 & (Aber et al. 1990) & \\
\hline Acer saccharum & 52.1 & (Pastor et al. 1984) & \\
\hline Acer saccharum & 83.3 & (Melillo et al. 1982) & \\
\hline Alnus glutinosa & 16.3 & (Bocock 1964) & \\
\hline Alnus rubra & 23.8 & (Aber and Melillo 1982) & \\
\hline Alnus rubra & 31.5 & (Edmonds 1980) & \\
\hline Alnus viridis & 44.1 & (Fyles and McGill 1987) & \\
\hline Betula & 54.3 & (Aber and Melillo 1980) & \\
\hline Betula alleghaniensis & 58.8 & (Gosz et al. 1973) & \\
\hline Betula Papyrifera & 55.6 & (Aber and Melillo 1982) & \\
\hline Betula Papyrifera & 55.6 & (Aber et al. 1990) & \\
\hline Betula Papyrifera & 55.6 & (Melillo et al. 1982) & \\
\hline Betula pendula & 64.9 & (Berg and Ekhbom 1991) & \\
\hline Betula pubescens & 65.8 & (Berg et al. 1984) & \\
\hline Carya spp. & 38.5 & (Aber and Melillo 1982) & \\
\hline Castanea dentata & 47.6 & (Aber and Melillo 1982) & \\
\hline Castanea sativa & 114 & (Cortez et al. 1996) & \\
\hline Castanea sativa & 64.9 & (Anderson 1973) & \\
\hline Ceanothus spp. & 58.8 & (Aber and Melillo 1982) & \\
\hline Cornus florida & 34.4 & (Aber and Melillo 1982) & \\
\hline Corylus avellana & 36.0 & (Bocock 1964) & \\
\hline Fagus & 55.6 & (Aber and Melillo 1980) & \\
\hline Fagus grandifolia & 61.0 & (Gosz et al. 1973) & \\
\hline Fagus grandifolia & 58.8 & (Aber and Melillo 1982) & \\
\hline Fagus grandifolia & 55.6 & (Melillo et al. 1982) & \\
\hline Fagus sylvatica & 64.0 & (Cortez et al. 1996) & \\
\hline Fagus sylvatica & 42.7 & (Bocock 1964) & \\
\hline Fagus sylvatica & 42.7 & (Anderson 1973) & \\
\hline Fraxinus & 49.5 & (Aber and Melillo 1980) & \\
\hline Fraxinus americana & 55.6 & (Aber and Melillo 1982) & \\
\hline Fraxinus americana & 50.0 & (Pastor et al. 1984) & \\
\hline Fraxinus americana & 55.6 & (Melillo et al. 1982) & \\
\hline Fraxinus angustifolia & 53.8 & (Gallardo and Merino 1993) & \\
\hline Fraxinus excelsior & 33.8 & (Gilbert and Bocock 1960) & \\
\hline Fraxinus excelsior & 32.3 & (Bocock 1964) & \\
\hline Liriodendron tulipifera & 45.5 & (Aber and Melillo 1982) & \\
\hline Nothofagus spp. & 66.0 & (Ross and Tate 1993) & \\
\hline Populus tremuloides & 70.8 & (Stump and Binkley 1993) & \\
\hline Populus tremuloides & 60.2 & (Aber et al. 1990) & \\
\hline Prunus & 43.5 & (Aber and Melillo 1980) & \\
\hline Prunus avium & 44.6 & (Bocock 1964) & \\
\hline Prunus pennsylvanica & 40.0 & (Aber and Melillo 1982) & \\
\hline Prunus pennsylvanica & 41.7 & (Melillo et al. 1982) & \\
\hline Quercus alba & 62.5 & (Aber and Melillo 1982) & \\
\hline Quercus alba & 59.5 & (Aber et al. 1990) & \\
\hline Quercus alba & 56.2 & (Pastor et al. 1984) & \\
\hline Quercus canariensis & 71.4 & (Gallardo and Merino 1993) & \\
\hline Quercus coccinea & 76.9 & (Aber and Melillo 1982) & \\
\hline Quercus ilex & 56.5 & (Cortez et al. 1996) & \\
\hline Quercus petraea & 63.9 & (Cortez et al. 1996) & \\
\hline Quercus petraea & 68.5 & (Bocock et al. 1960) & \\
\hline Quercus petraea & 54.3 & (Bocock 1963) & \\
\hline
\end{tabular}


Earth Interactions - Volume 4 (2000) - Paper No. 3 - Page 38

\begin{tabular}{lll}
\hline DBF & \multicolumn{2}{c}{ Mean $=55$, std dev $=16, n=76$} \\
\hline Quercus petraea & 64.9 & (Bocock 1964) \\
Quercus petraea & 64.9 & (Bocock 1964) \\
Quercus prinus & 41.7 & (Aber and Melillo 1982) \\
Quercus prinus/rubra & 48.1 & (Strojan 1978) \\
Quercus pyrenaica & 53.8 & (Gallardo and Merino 1993) \\
Quercus robur & 63.3 & (Bocock 1964) \\
Quercus rubra & 58.1 & (Gower and Son 1992) \\
Quercus rubra & 60.2 & (Aber et al. 1990) \\
Quercus rubra & 59.5 & (Aber et al. 1990) \\
Quercus rubra & 61.0 & (Aber et al. 1990) \\
Quercus rubra & 57.5 & (Pastor et al. 1984) \\
Quercus suber & 61.7 & (Gallardo and Merino 1993) \\
Robinia pseudoacacia & 32.2 & (Aber and Melillo 1982) \\
Salix atrocinerea & 68.5 & (Gallardo and Merino 1993) \\
Sassafras albidum & 35.2 & (Strojan 1978) \\
Tilia americana & 31.2 & (Pastor et al. 1984) \\
Grass & Mean & 45, std dev = 11, $n=10$ \\
\hline Dry alluvial meadow & 36.5 & (Titlyanova and Bazilevich 1979) \\
Grass & 43.7 & (Taylor et al. 1991) \\
Halophytic meadow- steppe & 51.5 & (Titlyanova and Bazilevich 1979) \\
Halophytic meadow & 38.5 & (Titlyanova and Bazilevich 1979) \\
Matador, Canada & 54.3 & (Coupland and van Dyne 1979) \\
Meadow-steppe & 51.5 & (Titlyanova and Bazilevich 1979) \\
Meadow-steppe & 35.7 & (Titlyanova and Bazilevich 1979) \\
Mesohalophytic meadow & 37.6 & (Titlyanova and Bazilevich 1979) \\
Steppe-meadow & 32.7 & (Titlyanova and Bazilevich 1979) \\
Wet halophytic meadow & 69.4 & (Titlyanova and Bazilevich 1979) \\
Shrub & Mean $=75$, std dev = 37, $n=11$ \\
\hline Alder & 26.4 & (Taylor et al. 1991) \\
Arctostaphylos & 66.7 & (Taylor et al. 1991) \\
Ceanothus megacarpus & 74.6 & (Schlessinger 1985) \\
Ceanothus megacarpus & 79.4 & (Schlessinger 1985) \\
Cistus libanotis & 122 & (Gallardo and Merino 1993) \\
Halimium halimifolium & 152 & (Gallardo and Merino 1993) \\
Quercus coccifera & 54.9 & (Gallardo and Merino 1993) \\
Quercus lusitanica & 54.9 & (Gallardo and Merino 1993) \\
Salvia mellifera & 86.2 & (Schlessinger 1985) \\
Salvia mellifera & 76.9 & (Schlessinger 1985) \\
Sepherdia & 27.9 & (Taylor et al. 1991) \\
\hline
\end{tabular}

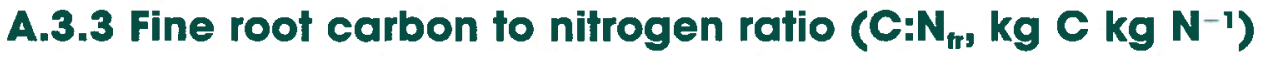

Fine root carbon to nitrogen ratio $\left(\mathrm{C}: \mathrm{N}_{\mathrm{fr}}, \mathrm{kg} \mathrm{C} \mathrm{kg} \mathrm{N}^{-1}\right.$ ) controls the nitrogen required for fine root construction, but has no effect on nutrient or water uptake.

\begin{tabular}{lll}
\hline ENF & \multicolumn{2}{c}{ Mean $=58$, std dev $=32, n=27$} \\
\hline Abies amabilis & 48.1 & (Grier et al. 1981; Vogt et al. 1982) \\
Abies amabilis & 54.9 & (Grier et al. 1981; Vogt et al. 1982) \\
Abies lasiocarpa & 81.5 & (Stump and Binkley 1993) \\
ENF & 59.2 & (Taylor et al. 1991) \\
ENF & 49.0 & (Vogt et al. 1986) \\
\hline
\end{tabular}


Earth Interactions - Volume 4 (2000) - Paper No. 3 - Page 39

\begin{tabular}{|c|c|c|}
\hline \multirow{2}{*}{$\frac{\text { ENF }}{\text { ENF }}$} & \multicolumn{2}{|c|}{ Mean $=58$, std dev $=32, n=27$} \\
\hline & 49.5 & (Vogt et al. 1986) \\
\hline ENF & 50.5 & (Vogt et al. 1986) \\
\hline ENF & 31.4 & (DeAngelis et al. 1981; Nadelhoffer et al. 1985) \\
\hline ENF & 36.5 & (DeAngelis et al. 1981; Nadelhoffer et al. 1985) \\
\hline ENF & 36.2 & (DeAngelis et al. 1981; Nadelhoffer et al. 1985) \\
\hline ENF & 50.0 & (DeAngelis et al. 1981; Nadelhoffer et al. 1985) \\
\hline ENF & 61.0 & $\begin{array}{l}\text { (Lutz and Cline 1947; McClaugherty et al. 1982; } \\
\text { Vogt et al. 1986) }\end{array}$ \\
\hline ENF & 40.3 & (Nambiar 1987) \\
\hline ENF & 42.4 & (Nambiar 1987) \\
\hline Picea engelmannii & 68.4 & (Stump and Binkley 1993) \\
\hline Picea/Abies & 27.6 & $\begin{array}{l}\text { (Kimmins and Hawkes 1978; Krumlik and Kim- } \\
\text { mins 1976) }\end{array}$ \\
\hline Picea/Abies & 37.0 & (Damman 1964; Damman 1971) \\
\hline Picea/Abies & 46.7 & (Damman 1964; Damman 1971) \\
\hline Pinus contorta & 82.4 & (Stump and Binkley 1993) \\
\hline Pinus strobus & 53.6 & (Aber et al. 1990) \\
\hline Pinus taeda & 61.7 & (Birk and Vitousek 1986) \\
\hline Pinus taeda & 49.5 & (Birk and Vitousek 1986) \\
\hline Pinus taeda & 48.5 & (Birk and Vitousek 1986) \\
\hline Pinus taeda & 52.6 & (Birk and Vitousek 1986) \\
\hline Pinus taeda & 54.9 & (Birk and Vitousek 1986) \\
\hline Pseudotsuga menziesii & 79.4 & (Grier et al. 1974; Santantonio et al. 1977) \\
\hline Pseudotsuga menziesii & 200 & (Grier et al. 1974; Santantonio et al. 1977) \\
\hline DNF & 58 & Set to ENF \\
\hline DBF & \multicolumn{2}{|c|}{ Mean $=48$, std dev $=15, n=16$} \\
\hline Acer saccharum & 29.9 & (Aber et al. 1990) \\
\hline DBF & 25.0 & (Fahey et al. 1978) \\
\hline DBF & 53.2 & (Yin 1989) \\
\hline DBF & 37.6 & (DeAngelis et al. 1981; Nadelhoffer et al. 1985) \\
\hline DBF & 42.0 & (DeAngelis et al. 1981; Nadelhoffer et al. 1985) \\
\hline DBF & 43.9 & (DeAngelis et al. 1981; Nadelhoffer et al. 1985) \\
\hline DBF & 42.7 & (DeAngelis et al. 1981; Nadelhoffer et al. 1985) \\
\hline DBF & 37.9 & (DeAngelis et al. 1981; Nadelhoffer et al. 1985) \\
\hline $\mathrm{DBF}$ & 37.9 & $\begin{array}{l}\text { (Lutz and Cline 1947; McClaugherty et al. 1984; } \\
\text { Vogt et al. 1986) }\end{array}$ \\
\hline DBF & 58.8 & $\begin{array}{l}\text { (Lutz and Cline 1947; McClaugherty et al. 1984; } \\
\text { Vogt et al. 1986) }\end{array}$ \\
\hline DBF & 46.7 & $\begin{array}{l}\text { (Lutz and Cline 1947; McClaugherty et al. 1982; } \\
\text { Vogt et al. 1986) }\end{array}$ \\
\hline Populus tremuloides & 52.4 & (Stump and Binkley 1993) \\
\hline Quercus & 36.2 & (Joslin and Henderson 1987) \\
\hline Quercus & 68.5 & (Joslin and Henderson 1987) \\
\hline Quercus & 75.8 & (Joslin and Henderson 1987) \\
\hline Quercus & 73.5 & (Joslin and Henderson 1987) \\
\hline Grass & \multicolumn{2}{|c|}{ Mean $=50$, std dev $=19, n=17$} \\
\hline Dry alluvial meadow & 61.0 & (Titlyanova and Bazilevich 1979) \\
\hline Grass & 48.0 & (Taylor et al. 1991) \\
\hline Halophytic meadow-Steppe & 70.4 & (Titlyanova and Bazilevich 1979) \\
\hline Halophytic meadow & 72.5 & (Titlyanova and Bazilevich 1979) \\
\hline Matador, Canada & 75.8 & (Coupland and van Dyne 1979) \\
\hline
\end{tabular}


Earth Interactions - Volume 4 (2000) - Paper No. 3 - Page 40

\begin{tabular}{llll}
\hline Grass & \multicolumn{2}{l}{ Mean $=50$, std dev $=19, n=17$} & (Continued) \\
\hline Meadow-steppe & 40.0 & (Titlyanova and Bazilevich 1979) & \\
Meadow-steppe & 62.5 & (Titlyanova and Bazilevich 1979) & \\
Mesohalophytic meadow & 21.7 & (Titlyanova and Bazilevich 1979) & \\
Mesohalophytic meadow & 45.9 & (Titlyanova and Bazilevich 1979) \\
Mesophytic alluvial meadow & 57.5 & (Titlyanova and Bazilevich 1979) \\
Mesophytic alluvial meadow & 33.1 & (Titlyanova and Bazilevich 1979) \\
Mesophytic meadow & 42.4 & (Titlyanova and Bazilevich 1979) \\
Solling Plateau & 34.0 & (Titlyanova and Bazilevich 1979) \\
Steppe-meadow & 22.3 & (Titlyanova and Bazilevich 1979) \\
Steppe-meadow & 37.9 & (Titlyanova and Bazilevich 1979) \\
Wet alluvial meadow & 37.3 & (Titlyanova and Bazilevich 1979) \\
Wet halophytic meadow & 87.7 & (Titlyanova and Bazilevich 1979) & \\
Shrub & 58 & Set to ENF & \\
\hline
\end{tabular}

\section{A.3.4 Live wood carbon to nitrogen ratio ( $C: \mathrm{N}_{\mathrm{Iw}}, \mathbf{k g ~ C ~} \mathbf{~ g ~ N ~ N}^{-1}$ )}

Limited data from small branches, which are mostly cambium, suggest that the live wood carbon to nitrogen ratio $\left(\mathrm{C}: \mathrm{N}_{\mathrm{lw}}, \mathrm{kg} \mathrm{C} \mathrm{kg} \mathrm{N}-1\right)$ is similar to $\mathrm{C}: \mathrm{N}_{\mathrm{fr}}$ (Gosz et al. 1973). Lacking data for $C: N_{l w}$ itself, we therefore set $C: N_{l w}$ to the mean $C$ : $\mathrm{N}_{\mathrm{fr}}$ rounded to one significant digit.

\section{A.3.5 Dead wood carbon to nitrogen ratio ( $\left.C: \mathrm{N}_{\mathrm{dw}}, \mathrm{kg} \mathrm{C} \mathrm{kg} \mathrm{N}^{-1}\right)$}

\begin{tabular}{lll}
\hline ENF & Mean $=730$, std dev $=320, n=27$ \\
\hline Abies & 212 & (Allison et al. 1963) \\
Abies amabilis & 680 & (Edmonds 1987) \\
Abies concolor & 996 & (Allison et al. 1963) \\
Calocedrus & 526 & (Allison et al. 1963) \\
Cedar & 365 & (Allison et al. 1963) \\
Cupressus & 882 & (Allison et al. 1963) \\
Larix occidentalis & 270 & (Allison et al. 1963) \\
Picea engelmannii & 411 & (Allison et al. 1963) \\
Pinus contorta & 660 & (Allison et al. 1963) \\
Pinus contorta & 1400 & (Fahey et al. 1985) \\
Pinus echinata & 346 & (Allison et al. 1963) \\
Pinus elliottii & 984 & (Allison et al. 1963) \\
Pinus lambertiana & 404 & (Allison et al. 1963) \\
Pinus monticola & 433 & (Allison et al. 1963) \\
Pinus palustris & 1310 & (Allison et al. 1963) \\
Pinus ponderosa & 867 & (Allison et al. 1963) \\
Pinus strobus & 555 & (Allison et al. 1963) \\
Pinus strobus & 1250 & (Berg et al. 1984) \\
Pinus taeda & 716 & (Allison et al. 1963) \\
Pseudotsuga menziesii & 943 & (Allison et al. 1963) \\
Pseudotsuga menziesii & 667 & (Aber and Melillo 1980) \\
Pseudotsuga menziesii & 1040 & (Edmonds 1987) \\
Sequoia & 822 & (Allison et al. 1963) \\
Tsuga canadensis & 458 & (Allison et al. 1963) \\
Tsuga heterophylla & 991 & (Edmonds 1987) \\
Tsuga/Picea & 769 & (Grier 1978) \\
Wood & 710 & (Harmon et al. 1986) \\
\hline & &
\end{tabular}


Earth Interactions - Volume 4 (2000) - Paper No. 3 - Page 41

\begin{tabular}{|c|c|c|c|}
\hline DNF & Set to ENF & & (Continued) \\
\hline $\mathrm{DBF}$ & \multicolumn{2}{|c|}{ Mean $=550$, std dev $=121, n=11$} & \\
\hline Acer & 556 & (McClaugherty et al. 1985) & \\
\hline Carya & 468 & (Allison et al. 1963) & \\
\hline Castanea & 654 & (Allison et al. 1963) & \\
\hline Eucalyptus & 819 & (Allison et al. 1963) & \\
\hline Juglans nigra & 470 & (Allison et al. 1963) & \\
\hline Liriodendron tulipifera & 535 & (Allison et al. 1963) & \\
\hline Quercus alba & 451 & (Allison et al. 1963) & \\
\hline Quercus rubra & 479 & (Allison et al. 1963) & \\
\hline Quercus stellata & 492 & (Allison et al. 1963) & \\
\hline Quercus velutina & 676 & (Allison et al. 1963) & \\
\hline Wood & 421 & (Harmon et al. 1986) & \\
\hline Grass & \multicolumn{2}{|c|}{ No woody component } & \\
\hline Shrub & 730 & Set to ENF & \\
\hline
\end{tabular}

\section{A.4 Labile, cellulose, and lignin parameters}

Each plant pool entering the soil decomposition subroutine is divided into three pools (two for dead wood): labile, cellulose, and lignin. The fractionation into these pools controls how rapidly decomposition occurs. In general, lab techniques are used to first measure the water and acid soluble material, which in addition to starch and sugar may include other substances, such as phenols. This is termed the labile pool. Next, cellulose is measured with an acid bath. The remainder is grouped into the lignin pool, which may include extraneous suberin (Wedin et al. 1995). Since the three pools may include different substances depending on the methodology in use, they should be considered as generalized categories, not pure labile material, cellulose, or lignin. Data sources in some cases had only one or two of the fractions listed and therefore mean biome values did not necessarily add to $100 \%$. We first calculated lignin and cellulose fractions and let the labile percent float so that the three pools summed to $100 \%$. For dead wood, we calculated lignin and floated cellulose.

\section{A.4.1 Fine root fractions}

For fine root fractions (percent), data sources were quite limited. Rather than set a biome value based on a single data point, we calculated the mean of all fine root data and used this for all biomes.

\begin{tabular}{|c|c|c|c|c|}
\hline \multirow[t]{2}{*}{ All biomes } & \multicolumn{4}{|c|}{$\begin{array}{l}\text { Labile: mean }=34, \text { std dev }=2.8, n=4 \\
\text { Cellulose: mean }=44, \text { std dev }=4.8, n=6 \\
\text { Lignin: } \text { mean }=22, \text { std } \operatorname{dev}=7.3, n=12\end{array}$} \\
\hline & Labile & Cellulose & Lignin & \\
\hline Abies lasiocarpa & & 37.8 & 19.8 & (Stump and Binkley 1993) \\
\hline Acer saccharum & 18.5 & 47.7 & 33.8 & (Taylor et al. 1991) \\
\hline Agropyron repens & & & 15.9 & (Wedin et al. 1995) \\
\hline Agrostis scabra & & & 9.50 & (Wedin et al. 1995) \\
\hline ENF & 23.3 & & 36.1 & (Taylor et al. 1991) \\
\hline
\end{tabular}




\begin{tabular}{|c|c|c|c|c|c|}
\hline \multirow[t]{2}{*}{ All biomes } & \multicolumn{4}{|c|}{$\begin{array}{l}\text { Labile: } \text { mean }=34, \text { std dev }=2.8, n=4 \\
\text { Cellulose: mean }=44, \text { std dev }=4.8, n=6 \\
\text { Lignin: mean }=22, \text { std dev }=7.3, n=12\end{array}$} & \multirow[t]{2}{*}{ (Continued) } \\
\hline & Labile & Cellulose & Lignin & & \\
\hline Grass & 22.2 & & 24.7 & (Taylor et al. 1991) & \\
\hline Picea Engelmannii & & 38.1 & 19.2 & (Stump and Binkley 1993) & \\
\hline Pinus contorta & & 43.3 & 21.4 & (Stump and Binkley 1993) & \\
\hline Pinus strobus & 25.2 & 49.5 & 25.3 & (Aber et al. 1990) & \\
\hline Poa pratensis & & & 17.0 & (Wedin et al. 1995) & \\
\hline Populus tremuloides & & 44.4 & 22.3 & (Stump and Binkley 1993) & \\
\hline Schizachyrium scopar. & & & 22.5 & (Wedin et al. 1995) & \\
\hline
\end{tabular}

\section{A.4.2 Litter fractions}

Litter fractions (percent) were better represented in the literature and we parameterized separate biomes.

\begin{tabular}{|c|c|c|c|c|}
\hline \multirow[t]{2}{*}{ ENF } & \multicolumn{4}{|c|}{$\begin{array}{l}\text { Labile: } \text { mean }=31, \text { std dev }=12, n=11 \\
\text { Cellulose: mean }=45, \text { standard dev }=4.7, n=7 \\
\text { Lignin: } \text { mean }=24, \text { std dev }=6.7, n=29\end{array}$} \\
\hline & Labile & Cellulose & Lignin & \\
\hline Abiea amabilis & & & 25.4 & (Edmonds 1984) \\
\hline Abiea balsamea & & & 27.6 & (Fyles and McGill 1987) \\
\hline Abies concolor & & & 17.0 & (Stohlgren 1988) \\
\hline Abies concolr & & & 16.2 & (Stohlgren 1988) \\
\hline Abies lasiocarpa & & 45.7 & 26.5 & (Stump and Binkley 1993) \\
\hline Abies lasiocarpa & 54.4 & & 14.6 & (Taylor et al. 1991) \\
\hline Calocedrus decurrens & & & 9.6 & (Stohlgren 198) \\
\hline Picea abies & & & 34.0 & (Berg and McClaugherty 1989) \\
\hline Picea engelmannii & & 49.1 & 26.1 & (Stump and Binkley 1993) \\
\hline Picea engelmannii & 48.9 & & 14.6 & (Taylor et al. 1991) \\
\hline Picea glauca & & & 24.9 & (Fyles and McGill 1987) \\
\hline Pinus banksiana & & & 29.3 & (Fyles and McGill 1987) \\
\hline Pinus contorta & & & 37.6 & (Berg and McClaugherty 1989) \\
\hline Pinus contorta & & 37.0 & 25.2 & (Stump and Binkley 1993) \\
\hline Pinus contorta & 14.5 & & 38.1 & (Berg and Ekhbom 1991) \\
\hline Pinus contorta & 32.5 & & 24.5 & (Taylor et al. 1991) \\
\hline Pinus elliottii & & & 23.7 & (Gholz et al. 1985) \\
\hline Pinus lambertiana & & & 18.3 & (Stohlgren 1988) \\
\hline Pinus lambertiana & & & 16.4 & (Stohlgren 1988) \\
\hline Pinus ponderosa & 18.7 & & 26.1 & (Hart et al. 1992) \\
\hline Pinus ponderosa & 21.4 & & 30.9 & (Hart et al. 1992) \\
\hline Pinus resinosa & 25.8 & 46.5 & 27.7 & (Aber et al. 1990) \\
\hline Pinus strobus & 32.8 & 44.7 & 22.5 & (Aber et al. 1990) \\
\hline Pinus sylvestris & 25.7 & 49.3 & 25.0 & (Berg et al. 1984) \\
\hline Pinus sylvestris & & & 28.6 & (Berg and McClaugherty 1989) \\
\hline Pinus sylvestris & 27.7 & & 23.1 & (Berg and Ekhbom 1991) \\
\hline Pseudotsuga menziesii & & & 24.0 & (Aber and Melillo 1982) \\
\hline Sequoiadendron giganteum & & & 20.3 & (Stohlgren 1988) \\
\hline Tsuga & 39.8 & 39.6 & 20.6 & (Aber et al. 1990) \\
\hline DNF & 31 & 45 & 24 & Set to ENF \\
\hline
\end{tabular}




\begin{tabular}{|c|c|c|c|c|c|}
\hline \multirow[t]{2}{*}{ DBF } & \multicolumn{4}{|c|}{$\begin{array}{l}\text { Labile: } \text { mean }=38, \text { std dev }=10, n=15 \\
\text { Cellulose: } \text { mean }=44, \text { standard dev }=11, n=20 \\
\text { Lignin: } \text { mean }=18, \text { std dev }=6.6, n=44\end{array}$} & \multirow[t]{2}{*}{ (Continued) } \\
\hline & Labile & Cellulose & Lignin & & \\
\hline Acer rubrum & & & 10.5 & (Aber and Melillo 1982) & \\
\hline Acer rubrum & 44.7 & 38.0 & 17.3 & (Aber et al. 1990) & \\
\hline Acer rubrum & 43.9 & 38.9 & 17.2 & (Aber et al. 1990) & \\
\hline Acer rubrum & & & 10.1 & (Melillo et al. 1982) & \\
\hline Acer saccharum & & & 10.5 & (Aber and Melillo 1982) & \\
\hline Acer saccharum & 44.8 & 43.1 & 12.1 & (Aber et al. 1990) & \\
\hline Acer saccharum & & & 10.1 & (Melillo et al. 1982) & \\
\hline Alnus rubra & & & 10.5 & (Aber and Melillo 1982) & \\
\hline Alnus viridis & & & 24.6 & (Fyles and McGill 1987) & \\
\hline Betula & 37.7 & 35.9 & 26.3 & (Berg et al. 1984) & \\
\hline Betula & 29.7 & & 33.0 & (Berg and Ekhbom 1991) & \\
\hline Betula papyrifera & & & 14.0 & (Aber and Melillo 1982) & \\
\hline Betula papyrifera & 41.7 & 37.6 & 20.1 & (Aber et al. 1990) & \\
\hline Betula papyrifera & & & 14.5 & (Melillo et al. 1982) & \\
\hline Carya & & & 17.0 & (Aber and Melillo 1982) & \\
\hline Castanea & & & 9.0 & (Aber and Melillo 1982) & \\
\hline Castanea sativa & 23.4 & 69.4 & 9.2 & (Cortez et al. 1996) & \\
\hline Ceanothus & & & 10.5 & (Aber and Melillo 1982) & \\
\hline Cornus florida & & & 6.0 & (Aber and Melillo 1982) & \\
\hline Fagus & & & 23.0 & (Aber and Melillo 1982) & \\
\hline Fagus & & & 24.1 & (Melillo et al. 1982) & \\
\hline Fagus sylvatica & 12.4 & 56.1 & 31.5 & (Cortez et al. 1996) & \\
\hline Fraxinus & & & 12.2 & (Melillo et al. 1982) & \\
\hline Fraxinus americana & & & 12.5 & (Aber and Melillo 1982) & \\
\hline Fraxinus angustifolia & & 29.5 & 10.5 & (Gallardo and Merino 1993) & \\
\hline Liriodendron tulipifera & & & 15.0 & (Aber and Melillo 1982) & \\
\hline Populus tremuloides & & 40.6 & 19.4 & (Stump and Binkley 1993) & \\
\hline Populus tremuloides & 31.1 & 47.5 & 21.4 & (Aber et al. 1990) & \\
\hline Prunus pensylvannica & & & 18.0 & (Aber and Melillo 1982) & \\
\hline Prunus pensylvannica & & & 19.3 & (Melillo et al. 1982) & \\
\hline Quercus alba & & & 17.0 & (Aber and Melillo 1982) & \\
\hline Quercus alba & 32.4 & 47.4 & 20.2 & (Aber et al. 1990) & \\
\hline Quercus canariensis & & 37.9 & 15.1 & (Gallardo and Merino 1993) & \\
\hline Quercus coccinea & & & 17.0 & (Aber and Melillo 1982) & \\
\hline Quercus ilex & 13.4 & 62.4 & 24.2 & (Cortez et al. 1996) & \\
\hline Quercus petraea & 21.1 & 56.0 & 24.0 & (Cortez et al. 1996) & \\
\hline Quercus prinus & & & 25.5 & (Aber and Melillo 1982) & \\
\hline Quercus pyrenaica & & 43.1 & 14.3 & (Gallardo and Merino 1993) & \\
\hline Quercus rubra & 30.8 & 42.5 & 26.7 & (Aber et al. 1990) & \\
\hline Quercus rubra & 28.4 & 43.4 & 28.2 & (Aber et al. 1990) & \\
\hline Quercus rubra & 30.0 & 45.2 & 24.8 & (Aber et al. 1990) & \\
\hline Quercus suber & & 42.1 & 18.1 & (Gallardo and Merino 1993) & \\
\hline Robinia pseudoacacia & & & 25.5 & (Aber and Melillo 1982) & \\
\hline Salix atrocinerea & & 22.4 & 18.1 & (Gallardo and Merino 1993) & \\
\hline \multirow[t]{4}{*}{ Grass } & \multirow{3}{*}{\multicolumn{5}{|c|}{$\begin{array}{l}\text { Labile: } \text { mean }=68, \text { std dev }=\text { NA, } n=1 \\
\text { Cellulose: } \text { mean }=23, \text { std dev }=7.7, n=7 \\
\text { Lignin: } \text { mean }=9.0, \text { std dev }=4.3, n=13\end{array}$}} \\
\hline & & & & & \\
\hline & & & & & \\
\hline & Labile & Cellulose & Lignin & & \\
\hline Agropyron repens & & & 12.5 & (Wedin et al. 1995) & \\
\hline Agrostis scabra & & & 17.4 & (Wedin et al. 1995) & \\
\hline
\end{tabular}


Earth Interactions - Volume 4 (2000) - Paper No. 3 - Page 44

\begin{tabular}{|c|c|c|c|c|}
\hline \multirow[t]{2}{*}{ Grass } & \multicolumn{4}{|c|}{$\begin{array}{l}\text { Labile: } \text { mean }=68, \text { std dev }=\text { NA, } n=1 \\
\text { Cellulose: mean }=23, \text { std dev }=7.7, n=7 \\
\text { Lignin: } \text { mean }=9.0, \text { std dev }=4.3, n=13\end{array}$} \\
\hline & Labile & Cellulose & Lignin & \\
\hline Dactylotaenium & & 32.0 & 7.8 & (Mtambanengwe and Kirchmann 1995) \\
\hline Grass & 30.0 & & 12.0 & (Taylor et al. 1991) \\
\hline Grass pasture & & & 12.0 & (Cadisch et al. 1996) \\
\hline Mixed grasses & & 16.2 & 5.3 & (Singer and Harter 1996) \\
\hline Mixed grasses & & 12.9 & 4.1 & (Singer and Harter 1996) \\
\hline Mixed grasses & & 17.0 & 5.4 & (Singer and Harter 1996) \\
\hline Mixed grasses & & 29.0 & 6.5 & (Singer and Harter 1996) \\
\hline Mixed grasses & & 29.6 & 6.1 & (Singer and Harter 1996) \\
\hline Mixed grasses & & 26.9 & 6.0 & (Singer and Harter 1996) \\
\hline Poa pratensis & & & 10.7 & (Wedin et al. 1995) \\
\hline Schizachyrium scoparium & & & 15.4 & (Wedin et al. 1995) \\
\hline \multirow{4}{*}{ Shrub } & \multirow{3}{*}{\multicolumn{4}{|c|}{$\begin{array}{l}\text { Labile: } \text { mean }=56, \text { std dev }=21, n=7 \\
\text { Cellulose: mean }=29, \text { std dev }=8.6, n=4 \\
\text { Lignin: } \text { mean }=15, \text { std dev }=6.1, n=16\end{array}$}} \\
\hline & & & & \\
\hline & & & & \\
\hline & Labile & Cellulose & Lignin & \\
\hline Alnus & 53.7 & & 16.2 & (Taylor et al. 1991) \\
\hline Bearberry & 58.1 & & 16.6 & (Taylor et al. 1991) \\
\hline Ceanothus megacarpus & 22.3 & & 19.8 & (Schlessinger 1985) \\
\hline Ceanothus megacarpus & 19.7 & & 31.2 & (Schlessinger 1985) \\
\hline Chilopsis linearis & & & 14.6 & (Schaefer et al. 1985) \\
\hline Cistus libanotis & & 17.4 & 8.8 & (Gallardo and Merino 1993) \\
\hline Flourensia cernua & & & 9.6 & (Schaefer et al. 1985) \\
\hline Halimium halimifolium & & 26.8 & 8.9 & (Gallardo and Merino 1993) \\
\hline Larrea tridentata & & & 10.6 & (Schaefer et al. 1985) \\
\hline Prosopis glandulosa & & & 7.9 & (Schaefer et al. 1985) \\
\hline Quercus coccifera & & 36.4 & 18.8 & (Gallardo and Merino 1993) \\
\hline Quercus lusitanica & & 34.1 & 20.1 & (Gallardo and Merino 1993) \\
\hline Salvia mellifera & 13.2 & & 15.6 & (Schlessinger 1985) \\
\hline Salvia mellifera & 14.3 & & 16.9 & (Schlessinger 1985) \\
\hline Shepherdia & 55.6 & & 9.2 & (Taylor et al. 1991) \\
\hline Yucca elata & & & 9.9 & (Schaefer et al. 1985) \\
\hline
\end{tabular}

\section{A.4.3 Dead wood fractions (percent)}

\begin{tabular}{|c|c|c|c|}
\hline \multirow[t]{2}{*}{ ENF } & \multicolumn{3}{|c|}{$\begin{array}{l}\text { Cellulose: } \text { mean }=71, \text { std dev }=1.9, n=16 \\
\text { Lignin: } \text { mean }=29, \text { std dev }=3.1, n=19\end{array}$} \\
\hline & Lignin & Cellulose & \\
\hline Abies balsamea & 70.0 & 29.5 & $\begin{array}{l}\text { (Clermont and Schwartz 1951; Côte } \\
\text { 1977; Panshin and de Zeeuw 1980; } \\
\text { Timmell 1957) }\end{array}$ \\
\hline Larix larcina & 72.0 & 28.0 & $\begin{array}{l}\text { (Panshin and de Zeeuw 1980; Timmell } \\
\text { 1957) }\end{array}$ \\
\hline Picea abies & 70.0 & 30.0 & (Rydholm 1965) \\
\hline Picea glauca & 70.5 & 28.5 & $\begin{array}{l}\text { (Clermont and Schwartz 1951; } \\
\text { Panshin and de Zeeuw 1980; Timmell } \\
\text { 1967; Timmell 1957) }\end{array}$ \\
\hline Picea mariana & 72.5 & 27.5 & $\begin{array}{l}\text { (Clermont and Schwartz 1951; Wise } \\
\text { and Jahn 1952) }\end{array}$ \\
\hline
\end{tabular}


Earth Interactions - Volume 4 (2000) - Paper No. 3 - Page 45

\begin{tabular}{|c|c|c|c|}
\hline \multirow[t]{2}{*}{ ENF } & \multicolumn{3}{|c|}{$\begin{array}{l}\text { Cellulose: } \text { mean }=71, \text { std dev }=1.9, n=16 \\
\text { Lignin: } \text { mean }=29, \text { std dev }=3.1, n=19\end{array}$} \\
\hline & Lignin & Cellulose & \\
\hline Pinus banksiana & 72.0 & 28.0 & $\begin{array}{l}\text { (Clermont and Schwartz 1951; } \\
\text { Timmell 1957) }\end{array}$ \\
\hline Pinus elliotii & 70.0 & 30.0 & (Panshin and de Zeeuw 1980) \\
\hline Pinus strobus & 72.5 & 27.5 & $\begin{array}{l}\text { (Clermont and Schwartz 1951; } \\
\text { Panshin and de Zeeuw 1980; Timmell } \\
\text { 1967) }\end{array}$ \\
\hline Pinus sylvestris & 70.0 & 30.0 & (Rydholm 1965) \\
\hline Pinus Taeda & 71.0 & 29.0 & (Wise and Jahn 1952) \\
\hline Pseudotsuga menziesii & 73.0 & 27.0 & $\begin{array}{l}\text { (Panshin and de Zeeuw 1980; Wise } \\
\text { and Jahn 1952) }\end{array}$ \\
\hline Sequoia sempervirens & 66.0 & 34.0 & (Panshin and de Zeeuw 1980) \\
\hline Thuja occidentalis & 68.0 & 32.0 & $\begin{array}{l}\text { (Côte 1977; Panshin and de Zeeuw } \\
\text { 1980; Timmell 1957) }\end{array}$ \\
\hline Tsuga canadensis & 68.0 & 32.0 & $\begin{array}{l}\text { (Clermont and Schwartz 1951; } \\
\text { Panshin and de Zeeuw 1980; Timmell } \\
\text { 1967; Timmell 1957) }\end{array}$ \\
\hline Tsuga heterophylla & 70.0 & 30.0 & (Wise and Jahn 1952) \\
\hline Pinus strobus & 68.3 & 22.1 & (Berg et al. 1984) \\
\hline Pseudotsuga menziesii & 22.8 & & (Edmonds 1987) \\
\hline Tsuga heterophylla & 25.2 & & (Edmonds 1987) \\
\hline Abies amabilis & 32.8 & & (Edmonds 1987) \\
\hline DNF & 71 & 29 & Set to ENF \\
\hline \multirow[t]{2}{*}{$\mathrm{DBF}$} & \multicolumn{3}{|c|}{$\begin{array}{l}\text { Cellulose: } \text { mean }=77, \text { std dev }=3.7, n=11 \\
\text { Lignin: } \text { mean }=23, \text { std dev }=4.9, n=11\end{array}$} \\
\hline & Lignin & Cellulose & \\
\hline Acer rubrum & 75.0 & 24.0 & (Côte 1977; Timmell 1957) \\
\hline Acer rubrum & 80.5 & 12.5 & (McClaugherty et al. 1985) \\
\hline Acer saccharum & 75.0 & 25.0 & (Panshin and de Zeeuw 1980) \\
\hline Betula lutea & 74.0 & 26.0 & (Panshin and de Zeeuw 1980) \\
\hline Betula papyrifera & 81.0 & 19.0 & $\begin{array}{l}\text { (Clermont and Schwartz 1951; } \\
\text { Timmell 1967; Timmell 1957) }\end{array}$ \\
\hline Betula verrucosa & 78.0 & 21.0 & (Rydholm 1965) \\
\hline Fagus grandifolia & 74.0 & 24.0 & $\begin{array}{l}\text { (Panshin and de Zeeuw 1980; Timmell } \\
\text { 1957) }\end{array}$ \\
\hline Populus tremuloides & 78.5 & 19.5 & $\begin{array}{l}\text { (Clermont and Schwartz 1951; Côte } \\
\text { 1977; Panshin and de Zeeuw 1980; } \\
\text { Timmell 1957) }\end{array}$ \\
\hline Quercus rubra & 75.0 & 25.0 & (Wise and Jahn 1952) \\
\hline Robinea pseudoacacia & 68.0 & 32.0 & (Panshin and de Zeeuw 1980) \\
\hline Ulmus americana & 74.0 & 24.0 & (Timmell 1967) \\
\hline Grass & \multicolumn{3}{|c|}{ No woody component } \\
\hline Shrub & 71 & 29 & Set to ENF \\
\hline
\end{tabular}

\section{A.5 Morphological parameters}

BIOME-BGC simulates the production of leaf carbon $\left(\mathrm{kg} \mathrm{C} \mathrm{m}{ }^{-2} \mathrm{day}^{-1}\right)$, which must be multiplied by specific leaf area to obtain LAI. The following parameters, 
including SLA, control how leaf carbon is morphologically distributed in the leaf and canopy.

\section{A.5.1 Specific leaf area}

LAI strongly influences all aspects of canopy physiology and is calculated as the product of SLA $\left(\mathrm{m}^{2} \mathrm{~kg} \mathrm{C}^{-1}\right)$ and leaf carbon $\left(\mathrm{kg} \mathrm{C} \mathrm{m}^{-2}\right)$. SLA defines leaf area per unit mass: thin, light leaves, such as grass blades, have a higher SLA than dense conifer needles. SLA is also used with C: $\mathrm{N}_{\text {leaf }}$ to calculate leaf nitrogen content on a per unit leaf area basis. Note that the definition of SLA is in mass units of $\mathrm{C}$ not dry weight (as almost always reported in the literature).

\begin{tabular}{lll}
\hline ENF & Mean $=8.2$, std dev $=$ 3.6, $n=39$ \\
\hline Abies grandis & 10.0 & (Gower and Richards 1990) \\
Juniperus virginia & 6.00 & (Reich et al. 1995a) \\
Picea abies & 7.80 & (Reich et al. 1995a) \\
Picea abies & 8.00 & (Gower and Richards 1990) \\
Picea abies & 6.80 & (Bauer et al. 1997) \\
Picea abies & 9.40 & (Bauer et al. 1997) \\
Picea abies & 9.40 & (Bauer et al. 1997) \\
Picea abies & 9.40 & (Bauer et al. 1997) \\
Picea abies & 6.40 & (Bauer et al. 1997) \\
Picea abies & 6.40 & (Bauer et al. 1997) \\
Picea abies & 6.80 & (Bauer et al. 1997) \\
Picea glauca & 7.00 & (Reich et al. 1995a) \\
Picea mariana & 7.40 & (Gower and Richards 1990) \\
Picea mariana & 9.76 & (Kloeppel et al. 1998) \\
Picea sitchensis & 9.80 & (Gower and Richards 1990) \\
Pinus albicaulis & 10.2 & (Gower and Richards 1990) \\
Pinus albicaulis & 7.58 & (Kloeppel et al. 1998) \\
Pinus banksiana & 8.20 & (Reich et al. 1995a) \\
Pinus contorta & 8.00 & (Gower 1987) \\
Pinus contorta & 7.60 & (Gower and Richards 1990) \\
Pinus contorta & 9.76 & (Kloeppel et al. 1998) \\
Pinus ponderosa & 2.40 & (Cregg 1994) \\
Pinus ponderosa & 2.08 & (Cregg 1994) \\
Pinus ponderosa & 2.01 & (Cregg 1994) \\
Pinus ponderosa & 2.44 & (Cregg 1994) \\
Pinus ponderosa & 2.25 & (Cregg 1994) \\
Pinus resinosa & 8.00 & (Reich et al. 1995a) \\
Pinus resinosa & 5.60 & (Reich et al. 1995a) \\
Pinus resinosa & 10.0 & (Gower and Richards 1990) \\
Pinus strobus & 11.4 & (Reich et al. 1995a) \\
Pinus strobus & 14.8 & (Reich et al. 1995a) \\
Pinus strobus & 14.8 & (Gower and Richards 1990) \\
Pinus sylvestris & 6.80 & (Reich et al. 1995a) \\
Pseudotsuga menziesii & 9.00 & (Gower 1987) \\
Pseudotsuga menziesii & 8.74 & (Kloeppel et al. 1998) \\
Pseudotsuga menziesii & 8.76 & (Kloeppel et al. 1998) \\
Thuja occidentalis & 9.00 & (Reich et al. 1995a) \\
Tsuga heterophylla & 21.0 & (Gower and Richards 1990) \\
Tsuga mertensiana & 9.20 & (Gower and Richards 1990) \\
\hline & &
\end{tabular}


Earth Interactions - Volume 4 (2000) - Paper No. 3 - Page 47

\begin{tabular}{|c|c|c|c|}
\hline \multirow{2}{*}{$\frac{\mathrm{DNF}}{\text { Larix decidua }}$} & \multicolumn{2}{|c|}{ Mean $=22$, std dev $=4.2, n=15$} & \multirow[t]{2}{*}{ (Continued) } \\
\hline & 24.6 & (Gower and Richards 1990) & \\
\hline Larix decidua & 16.0 & (Matyssek and Schulze 1987) & \\
\hline Larix eurolepsis & 17.9 & (Matyssek and Schulze 1987) & \\
\hline Larix laricina & 23.2 & (Gower and Richards 1990) & \\
\hline Larix laricina & 24.8 & (Kloeppel et al. 1998) & \\
\hline Larix leptolepsis & 28.2 & (Gower and Richards 1990) & \\
\hline Larix leptolepsis & 18.8 & (Matyssek and Schulze 1987) & \\
\hline Larix lyallii & 26.4 & (Gower and Richards 1990) & \\
\hline Larix lyallii & 21.2 & (Richards 1981) & \\
\hline Larix occidentalis & 16.8 & (Gower 1987) & \\
\hline Larix occidentalis & 15.2 & (Gower and Richards 1990) & \\
\hline Larix occidentalis & 26.4 & (Kloeppel et al. 1998) & \\
\hline Larix occidentalis & 22.8 & (Kloeppel et al. 1998) & \\
\hline Larix occidentalis & 24.0 & (Kloeppel et al. 1998) & \\
\hline Larix occidentalis & 25.6 & (Kloeppel et al. 1998) & \\
\hline DBF & \multicolumn{2}{|c|}{ Mean $=32$, std dev $=11, n=96$} & \\
\hline Acer negundo & 44.4 & (Abrams et al. 1994) & \\
\hline Acer rubrum & 33.2 & (Reich et al. 1995a) & \\
\hline Acer rubrum & 46.6 & (Reich et al. 1995a) & \\
\hline Acer saccharum & 26.6 & (Reich et al. 1995a) & \\
\hline Acer saccharum & 23.6 & (Reich et al. 1995a) & \\
\hline Acer saccharum & 52.6 & (Abrams et al. 1994) & \\
\hline Acer saccharum & 36.6 & (Burton et al. 1991) & \\
\hline Acer saccharum & 44.0 & (Burton et al. 1991) & \\
\hline Acer saccharum & 35.4 & (Burton et al. 1991) & \\
\hline Acer saccharum & 34.4 & (Burton et al. 1991) & \\
\hline Acer saccharum & 42.8 & (Burton et al. 1991) & \\
\hline Acer saccharum & 31.1 & (Jose and Gillespie 1996) & \\
\hline Betula lenta & 44.4 & (Abrams et al. 1994) & \\
\hline Betula nigra & 23.6 & (Reich et al. 1995a) & \\
\hline Betula pendula & 33.3 & (Kull and Niinemets 1993) & \\
\hline Betula pumila & 19.6 & (Reich et al. 1995a) & \\
\hline Beureria cumanensis & 23.3 & (Holbrook et al. 1995) & \\
\hline Bulnesia arborea & 22.0 & (Holbrook et al. 1995) & \\
\hline Carya cordiformis & 50.0 & (Abrams et al. 1994) & \\
\hline Carya glabra & 26.9 & (Jose and Gillespie 1996) & \\
\hline Carya ovata & 22.4 & (Reich et al. 1995a) & \\
\hline Castanea dentata & 34.5 & (Abrams et al. 1994) & \\
\hline Celtis occidentalis & 24.2 & (Reich et al. 1995a) & \\
\hline Celtis tenuifolia & 23.5 & (Abrams et al. 1994) & \\
\hline Coccoloba liebmannii & 25.0 & (Holbrook et al. 1995) & \\
\hline Cochlospermum vitifolium & 37.0 & (Holbrook et al. 1995) & \\
\hline Cornus alternifolia & 26.7 & (Abrams et al. 1994) & \\
\hline Cornus florida & 21.6 & (Reich et al. 1995a) & \\
\hline Fagus grandifolia & 29.3 & (Jose and Gillespie 1996) & \\
\hline Fagus sylvatica & 27.8 & (Bauer et al. 1997) & \\
\hline Fagus sylvatica & 48.0 & (Bauer et al. 1997) & \\
\hline Fagus sylvatica & 37.0 & (Bauer et al. 1997) & \\
\hline Fagus sylvatica & 37.8 & (Bauer et al. 1997) & \\
\hline Fagus sylvatica & 38.8 & (Bauer et al. 1997) & \\
\hline Forchhammeria pallida & 28.6 & (Holbrook et al. 1995) & \\
\hline Fraxinus americana & 26.4 & (Reich et al. 1995a) & \\
\hline
\end{tabular}




\section{Earth Interactions - Volume 4 (2000) - Paper No. 3 - Page 48}

\begin{tabular}{|c|c|c|c|}
\hline \multirow{2}{*}{$\frac{\mathrm{DBF}}{\text { Fraxinus americana }}$} & \multicolumn{2}{|c|}{ Mean $=32$, std dev $=11, n=96$} & \multirow[t]{2}{*}{ (Continued) } \\
\hline & 27.6 & (Reich et al. 1995a) & \\
\hline Fraxinus americana & 30.8 & (Abrams et al. 1994) & \\
\hline Fraxinus nigra & 52.6 & (Abrams et al. 1994) & \\
\hline Genipa caruto & 20.8 & (Holbrook et al. 1995) & \\
\hline Godmania macrocarpa & 16.3 & (Holbrook et al. 1995) & \\
\hline Humboldtiella arborea & 54.1 & (Holbrook et al. 1995) & \\
\hline Ilex verticillata & 24.4 & (Reich et al. 1995a) & \\
\hline Jacquinia pungens & 20.0 & (Holbrook et al. 1995) & \\
\hline Juglans nigra & 61.0 & (Reich et al. 1995a) & \\
\hline Juglans nigra & 30.8 & (Abrams et al. 1994) & \\
\hline Liriodendron tulipifera & 20.5 & (Jose and Gillespie 1996) & \\
\hline Lonchocarpus dipteroneurus & 48.8 & (Holbrook et al. 1995) & \\
\hline Lonicera $x$ bella & 21.8 & (Reich et al. 1995a) & \\
\hline Luehea candida & 46.5 & (Holbrook et al. 1995) & \\
\hline Mansoa verrucifera & 35.1 & (Holbrook et al. 1995) & \\
\hline Morus rubra & 36.4 & (Reich et al. 1995a) & \\
\hline Pereskia guamacho & 37.0 & (Holbrook et al. 1995) & \\
\hline Pithecellobium carabobense & 23.5 & (Holbrook et al. 1995) & \\
\hline Pithecellobium dulce & 30.3 & (Holbrook et al. 1995) & \\
\hline Pithecellobium ligustrinum & 30.8 & (Holbrook et al. 1995) & \\
\hline Populus deltoides & 21.8 & (Reich et al. 1995a) & \\
\hline Populus hybrid & 24.8 & (Heilman and Fu-Guang 1994) & \\
\hline Populus hybrid & 25.2 & (Heilman and Fu-Guang 1994) & \\
\hline Populus hybrid & 22.4 & (Heilman and Fu-Guang 1994) & \\
\hline Populus hybrid & 26.6 & (Heilman and Fu-Guang 1994) & \\
\hline Populus hybrid & 24.4 & (Heilman and Fu-Guang 1994) & \\
\hline Populus hybrid & 21.4 & (Heilman and Fu-Guang 1994) & \\
\hline Populus hybrid & 32.0 & (Heilman and Fu-Guang 1994) & \\
\hline Populus hybrid & 29.0 & (Heilman and Fu-Guang 1994) & \\
\hline Populus hybrid & 27.4 & (Heilman and Fu-Guang 1994) & \\
\hline Populus hybrid & 29.2 & (Heilman and Fu-Guang 1994) & \\
\hline Populus hybrid & 30.0 & (Heilman and Fu-Guang 1994) & \\
\hline Populus hybrid & 23.4 & (Heilman and Fu-Guang 1994) & \\
\hline Populus tremuloides & 24.2 & (Reich et al. 1995a) & \\
\hline Prunus pensylvanica & 30.8 & (Abrams et al. 1994) & \\
\hline Prunus serotina & 19.8 & (Reich et al. 1995a) & \\
\hline Prunus serotina & 50.0 & (Reich et al. 1995a) & \\
\hline Prunus serotina & 40.0 & (Abrams et al. 1994) & \\
\hline Prunus serotina & 26.7 & (Jose and Gillespie 1996) & \\
\hline Quercus alba & 20.4 & (Jose and Gillespie 1996) & \\
\hline Quercus ellipsoidalis & 19.0 & (Reich et al. 1995a) & \\
\hline Quercus ellipsoidalis & 37.0 & (Reich et al. 1995a) & \\
\hline Quercus macrocarpa & 22.8 & (Reich et al. 1995a) & \\
\hline Quercus macrocarpa & 33.3 & (Abrams et al. 1994) & \\
\hline Quercus prinus & 19.9 & (Jose and Gillespie 1996) & \\
\hline Quercus rubra & 26.2 & (Reich et al. 1995a) & \\
\hline Quercus rubra & 27.0 & (Reich et al. 1995a) & \\
\hline Quercus rubra & 20.2 & (Jose and Gillespie 1996) & \\
\hline Quercus velutina & 25.0 & (Abrams et al. 1994) & \\
\hline Randia aculeata & 60.6 & (Holbrook et al. 1995) & \\
\hline Rhamnus cathartica & 22.0 & (Reich et al. 1995a) & \\
\hline Rubus allighaniensis & 54.0 & (Reich et al. 1995a) & \\
\hline Salix dasyclados & 27.2 & (Kull et al. 1998) & \\
\hline
\end{tabular}




\begin{tabular}{|c|c|c|c|}
\hline \multirow{2}{*}{$\frac{\mathrm{DBF}}{\text { Salix viminalis }}$} & \multicolumn{2}{|c|}{ Mean $=32$, std dev $=11, n=96$} & \multirow[t]{2}{*}{ (Continued) } \\
\hline & 30.1 & (Kull et al. 1998) & \\
\hline Sassafras albidum & 30.8 & (Abrams et al. 1994) & \\
\hline Tabebuia billergiana & 38.5 & (Holbrook et al. 1995) & \\
\hline Tillia americana & 66.7 & (Abrams et al. 1994) & \\
\hline Tropical deciduous forest & 36.4 & (Maass et al. 1995) & \\
\hline Ulmus americana & 16.8 & (Reich et al. 1995a) & \\
\hline Ulmus rubra & 22.2 & (Abrams et al. 1994) & \\
\hline Grass & \multicolumn{2}{|c|}{ Mean $=49$, std dev $=16, n=35$} & \\
\hline Aegilops ovata & 46.2 & (Garnier et al. 1997) & \\
\hline Agropyron sp. & 55.0 & (Gamier et al. 1997) & \\
\hline Andropogon gerardii & 32.8 & (Knapp 1985) & \\
\hline Andropogon gerardii & 41.5 & (Knapp 1985) & \\
\hline Avena barbata & 50.8 & (Garnier et al. 1997) & \\
\hline Avenula bromoides & 36.8 & (Garnier et al. 1997) & \\
\hline Brachypodium distachyon & 65.6 & (Gamier et al. 1997) & \\
\hline Brachypodium phoenicoides & 31.4 & (Garnier et al. 1997) & \\
\hline Brachypodium phoenicoides & 33.2 & (Garnier et al. 1997) & \\
\hline Brachypodium retusum & 35.4 & (Garnier et al. 1997) & \\
\hline Brachypodium retusum & 36.8 & (Garnier et al. 1997) & \\
\hline Bromus erectus & 34.0 & (Garnier et al. 1997) & \\
\hline Bromus erectus & 40.4 & (Garnier et al. 1997) & \\
\hline Bromus erectus & 44.0 & (Garnier et al. 1997) & \\
\hline Bromus hordeaceus & 56.4 & (Garnier et al. 1997) & \\
\hline Bromus lanceolatus & 57.6 & (Garnier et al. 1997) & \\
\hline Bromus madritensis & 74.0 & (Garnier et al. 1997) & \\
\hline Bromus madritensis & 71.6 & (Garnier et al. 1997) & \\
\hline Dactylis glomerata & 40.8 & (Garnier et al. 1997) & \\
\hline Dactylis glomerata & 43.6 & (Garnier et al. 1997) & \\
\hline Desmazeria rigida & 46.6 & (Garnier et al. 1997) & \\
\hline Dichantium ischaemum & 58.0 & (Garnier et al. 1997) & \\
\hline Grass & 15.8 & (McWilliam et al. 1993) & \\
\hline Hordeum murinum & 58.8 & (Garnier et al. 1997) & \\
\hline Lolium rigidum & 50.6 & (Garnier et al. 1997) & \\
\hline Melica ciliata & 49.8 & (Garnier et al. 1997) & \\
\hline Melica ciliata & 43.0 & (Garnier et al. 1997) & \\
\hline Panicum virgatum & 37.1 & (Knapp 1985) & \\
\hline Panicum virgatum & 39.0 & (Knapp 1985) & \\
\hline Paspalum dilatatum & 66.0 & (Clark et al. 1997) & \\
\hline Phleum pratense & 54.2 & (Garnier et al. 1997) & \\
\hline Trifolium repens & 50.0 & (Clark et al. 1997) & \\
\hline Trifolium repens & 68.0 & (Clark et al. 1997) & \\
\hline Trifolium repens & 88.0 & (Clark et al. 1997) & \\
\hline Vulpia ciliata & 75.4 & (Garnier et al. 1997) & \\
\hline Shrub & \multicolumn{2}{|c|}{ Mean $=12$, std dev $=5.1, n=9$} & \\
\hline Gaultheria antipoda & 17.6 & (Körner et al. 1986) & \\
\hline Gaultheria antipoda & 18.1 & (Körner et al. 1986) & \\
\hline Gaultheria depressa & 14.7 & (Körner et al. 1986) & \\
\hline Gaultheria depressa & 14.6 & (Körner et al. 1986) & \\
\hline Ledum palustre & 5.62 & (Kudo 1995) & \\
\hline Ledum palustre & 11.4 & (Kudo 1995) & \\
\hline Ledum palustre & 13.4 & (Kudo 1995) & \\
\hline Pernettya alpina & 13.6 & (Körner et al. 1986) & \\
\hline Retama sphaerocarpa & 3.00 & (Pugnaire et al. 1996) & \\
\hline
\end{tabular}




\section{A.5.2 All-sided to projected leaf area index ratio}

Most canopy processes are estimated on a projected leaf area basis (the leaf area projected horizontally on the ground surface). Canopy water interception, though, is calculated under the assumption that all leaf surfaces retain water. Projected LAI therefore must be converted to all-sided LAI with $\mathrm{LAI}_{\text {all:proj }}\left(\mathrm{LAI} \mathrm{LAI}^{-1}\right)$. For flat leaves (grass and DBF) LAI $_{\text {all:proj }}$ is 2.0 and is not referenced. Needles are not flat and all-sided leaf area is greater. Accurate measurement of $L^{2 A I_{\text {all:proj }}}$ can be made by microscopic analysis of needle cross-sectional perimeter divided by maximum width (Cregg 1994) or by geometric approximations (Fassnacht et al. 1994). The mean value of 2.6 agrees with the general conifer value reported by Körner (Körner 1995). We assumed that shrub leaves were intermediate in shape and set shrub $L \mathrm{I}_{\text {all:proj }}$ to 2.3 .

\begin{tabular}{lll}
\hline ENF & \multicolumn{3}{c}{ Mean $=2.6$, std dev $=0.29, n=11$} \\
\hline Pinus ponderosa & 2.45 & (Cregg 1994) \\
Pinus ponderosa & 2.40 & (Cregg 1994) \\
Pinus ponderosa & 2.37 & (Cregg 1994) \\
Pinus ponderosa & 2.37 & (Cregg 1994) \\
Pinus ponderosa & 2.44 & (Cregg 1994) \\
Pinus contorta & 2.57 & (Barclay 1998) \\
Pinus strobus & 2.54 & (Swank and Schreuder 1973) \\
Pinus & 3.14 & (Deblonde et al. 1994) \\
Pinus & 3.14 & (Fassnacht et al. 1994) \\
Pinus & 2.57 & (Fassnacht et al. 1994) \\
Pinus & 2.30 & (Drew and Running 1975) \\
DNF & 2.6 & Set to ENF \\
\hline DBF & Defined as 2.0 \\
\hline Grass & Defined as 2.0 \\
\hline \multirow{2}{*}{ Shrub } & \multicolumn{2}{|}{} \\
\hline
\end{tabular}

\section{A.5.3 Shaded to sunlit specific leaf area ratio}

Nonlinear physiological responses to absorbed radiation tend to produce significant errors in predicted carbon and water fluxes in big leaf models (de Pury and Farquhar 1997) such as the original FOREST-BGC (Running and Coughlan 1988; Running and Gower 1991). Simulating multiple canopy layers obviates this problem, but is complicated and computationally expensive. De Pury and Farquhar (de Pury and Farquhar 1997) found that a two-layer model with sunlit and shaded portions solves most of the big leaf problems without excessive complexity and Thornton (Thornton 1998) describes the implementation of this approach in BIOME-BGC. Essentially, leaf nitrogen on a mass basis tends to stay relatively constant with canopy depth (Ellsworth and Reich 1993), but SLA increases, necessitating different SLAs for sunlit and shaded canopy fractions. Poorter and Evans (Poorter and Evans 1998) found that for a variety of shrub, tree, and herbaceous species, SLA in low irradiance was approximately twice the SLA in high irradiance while mass-based Rubisco content was essentially constant. We assigned 2.0 for SLA $_{\text {shd:sun }}\left(\mathrm{SLA} \mathrm{SLA}^{-1}\right)$ for all biomes. 
Earth Interactions - Volume 4 (2000) - Paper No. 3 - Page 51

\section{A.6 Conductance rates and limitations}

Leaf gas exchange is modeled through an electrical circuit analogy (Nobel 1991) with stomatal and cuticular conductance in parallel and leaf boundary layer conductance in series. The parameters controlling leaf conductance are important for regulating water loss and carbon assimilation. In this section, we consider the parameterization of maximum stomatal conductance, cuticular conductance, boundary layer conductance, and the two main parameters limiting stomatal aperture: leaf water potential and vapor pressure deficit.

\section{A.6.1 Maximum stomatal conductance}

Maximum stomatal conductance $\left(g_{\mathrm{smax}}, \mathrm{m} \mathrm{s}^{-1}\right)$ establishes the rate of conductance (g) when environmental conditions are nonlimiting. Major differences do exist between agricultural and natural vegetation, but within natural vegetation types, the major functional types are remarkably similar. Three reviews (Kelliher et al. 1995; Körner 1995; Schulze et al. 1994) all reached the same conclusion: $g_{\text {smax }}$ does not vary significantly between natural vegetation types. There is some discussion that grasslands may have higher $g_{\text {smax }}$ (Körner 1995), but to date there is insufficient evidence to establish this position. Thus, overwhelming evidence in this case led us to assign a single value for each biome. We adopted the most recent estimate, from Kelliher et al. (Kelliher et al. 1995), of $0.006 \mathrm{~m} \mathrm{~s}^{-1}$ for all biomes.

\section{A.6.2 Cuticular conductance}

Even when stomata are completely closed, gas exchange will still take place at very low rates through cuticular conductance $\left(g_{\text {cut }}, \mathrm{m} \mathrm{s}^{-1}\right)$. Essentially, leaf cuticles are somewhat leaky to gas exchange. Unfortunately, accurate measurements of $g_{\text {cut }}$ are rare and often inaccurate (Körner 1995). Measurement of $g_{\text {cut }}$ in the field is limited because plants almost never reach complete stomatal closure. Laboratory measurements of $g_{\text {cut }}$ almost always obtain lower values than $g_{\text {cut }}$ in the field. Measurements of $g_{\text {cut }}$ relevant for BIOME-BGC, at which stomata are completely closed, are almost impossible to obtain in the field. We therefore applied the lowest level reported by Körner (Körner 1995) of 1/100th of $g_{\text {smax }}$, or $0.00006 \mathrm{~m} \mathrm{~s}^{-1}$.

\section{A.6.3 Boundary layer conductance}

Boundary layer conductance $\left(g_{\mathrm{b}}, \mathrm{m} \mathrm{s}^{-1}\right)$ controls gas diffusion through the stable boundary layer around the leaf surface $\left(\delta_{\mathrm{b} 1}\right)$. Increases in leaf length in the wind direction increase $\delta_{\mathrm{bl}}$ according to a power function; increases in wind speed exponentially decrease $\delta_{\mathrm{bl}}$. Wind speed is not prescribed in BIOME-BGC and we assumed $0.45 \mathrm{~m} \mathrm{~s}^{-1}$. Fitting a curve to data in Nobel (Nobel 1991), $\delta_{\mathrm{bl}}=5.9574$ $\mathrm{L}^{0.4982}$, where $\delta_{\mathrm{bl}}$ is leaf boundary layer thickness $(\mathrm{mm})$ and $L$ is leaf length in the wind direction (m). For BIOME-BGC, $L=0.002 \mathrm{~m}$ and $\delta_{\mathrm{bl}}=0.27 \mathrm{~mm}$ (needle leaf), $L=0.08 \mathrm{~m}$ and $\delta_{\mathrm{bl}}=1.7$ (broad leaf), $L=0.01 \mathrm{~m}$ and $\delta_{\mathrm{bl}}=0.60 \mathrm{~mm}$ (grass), and $L=0.04 \mathrm{~m}$ and $\delta_{\mathrm{bl}}=1.2 \mathrm{~mm}$ (shrub). Following Nobel (Nobel 1991) boundary layer conductance is $g_{\mathrm{bl}}=D_{\mathrm{wv}} \delta_{\mathrm{bl}}$, where $D_{\mathrm{wv}}$ is the diffusion coefficient of water vapor in air $\left(2.4 \times 10^{-5} \mathrm{~m}^{2} \mathrm{~s}^{-1}\right.$ at $0.103 \mathrm{MPa}$ and $20^{\circ} \mathrm{C}$ ). Calculated values were 0.09 (ENF and DNF), 0.01 (DBF), 0.04 (grass), and 0.02 (shrub). 


\section{A.6.4 Leaf water potential at initial and final reduction to stomatal conductance}

Plant water stress, as measured in negative leaf water potential $\left(\Psi_{1}\right)$, is a commonly observed cause of stomatal closure. As leaf water potential drops below critical levels, leaf desiccation occurs, guard cell turgor is lost, and stomatal closure occurs. Diurnal plots can show a strong correlation between $g$ and $\Psi_{1}$ (Carlson et al. 1979). However, as seen in cases where maximal $g$ occurs at minimal $\Psi_{1}$ (Gallego et al. 1994; Hacke and Sauter 1995; Koch et al. 1994), there is no unique relationship between $\Psi_{1}$ and $g$. Other factors, such as vapor pressure deficit (VPD), directly influence the diurnal values of $\Psi_{1}$. Diurnal plots of $\Psi_{1}$ versus $g$ are therefore not useful for the parameterization. As shown by (Running 1976), the predawn leaf water potential $\left(\Psi_{\mathrm{lpd}}\right)$ is highly correlated with $g_{s \max }$. In this case, the plant is responding to long-term changes in soil water potential $\left(\Psi_{\mathrm{s}}\right)$, not daily variation in $\Psi_{1}$. Given no transpiration during the night, $\Psi_{\mathrm{lpd}}$ is usually approximately equal to $\Psi_{\mathrm{s}}$ (Kozlowski et al. 1991). Thus, $\Psi_{\mathrm{lpd}}$ acts as a surrogate measure for $\Psi_{s}$.

We calculated two parameters: the $\Psi_{\mathrm{lpd}}$ at which initial reduction to stomatal conductance occurs $\left(\Psi_{\mathrm{i}}\right)$ and the $\Psi_{\mathrm{lpd}}$ at which final reduction to stomatal conductance occurs $\left(\Psi_{\mathrm{f}}\right)$, that is, compete stomatal closure. In most cases, $\Psi_{\mathrm{i}}$ and $\Psi_{\mathrm{f}}$ were not reported and we subjectively extracted parameter values from scatterplots.

\begin{tabular}{|c|c|c|c|}
\hline \multirow[t]{2}{*}{ ENF } & \multicolumn{3}{|c|}{$\begin{array}{l}\text { Initial: mean }=-0.65, \text { std dev }=0.23, n=15 \\
\text { Final: mean }=-2.5, \text { std dev }=1.0, n=15\end{array}$} \\
\hline & Initial & Final & \\
\hline Abies bornmulleriana & -0.20 & -2.0 & (Guehl 1991) \\
\hline Cedrus atlantica & -0.50 & -3.3 & (Guehl 1991) \\
\hline Juniperus monosperma & -0.50 & -2.8 & (Lajtha and Barnes 1991) \\
\hline Juniperus osteosperma & -1.0 & -3.0 & (DeLucia and Schlesinger 1990) \\
\hline Picea glauca & -0.50 & -1.5 & (Eastman and Camm 1995) \\
\hline Picea glauca & -0.60 & -1.8 & (Goldstein et al. 1985) \\
\hline Picea mariana & -0.50 & -4.0 & (Dang et al. 1997) \\
\hline Pinus banksiana & -1.0 & -2.8 & (Dang et al. 1997) \\
\hline Pinus contorta & -0.50 & -1.4 & (Running 1980) \\
\hline Pinus edulis & -1.0 & -5.0 & (Lajtha and Barnes 1991) \\
\hline Pinus jeffreyi & -0.70 & -2.0 & (DeLucia and Schlesinger 1990) \\
\hline Pinus monophylla & -0.70 & -2.0 & (DeLucia and Schlesinger 1990) \\
\hline Pinus ponderosa & -0.80 & -1.5 & (Cregg 1994) \\
\hline Pinus ponderosa & -0.50 & -2.2 & (DeLucia et al. 1988) \\
\hline Pinus ponderosa & -0.70 & -2.0 & (DeLucia and Schlesinger 1990) \\
\hline DNF & -0.65 & -2.5 & Set to ENF \\
\hline \multirow[t]{2}{*}{ DBF } & \multicolumn{3}{|c|}{$\begin{array}{l}\text { Initial: mean }=-0.34, \text { std dev }=0.14, n=11 \\
\text { Final: mean }=-2.2, \text { std dev }=0.70, n=11\end{array}$} \\
\hline & Initial & Final & \\
\hline Acer saccharum & -0.20 & -1.3 & (Ni and Pallardy 1991) \\
\hline Juglans nigra & -0.20 & -1.5 & (Ni and Pallardy 1991) \\
\hline Quercus afares & -0.50 & -2.0 & (Acherar and Rambal 1992) \\
\hline Quercus alba & -0.20 & -2.0 & (Ni and Pallardy 1991) \\
\hline Quercus faginea & -0.50 & -2.0 & (Acherar and Rambal 1992) \\
\hline
\end{tabular}


Earth Interactions - Volume 4 (2000) - Paper No. 3 - Page 53

\begin{tabular}{llll}
\hline DBF & \multicolumn{4}{l}{ Initial: mean $=-0.34$, std dev $=0.14, n=11$} \\
& \multicolumn{4}{l}{ Final: mean $=-2.2$, std dev $=0.70, n=11$} & (Continued) \\
\hline & Initial & Final & \\
Quercus marilandica & -0.50 & -3.5 & (Reich and Hinckley 1980) \\
Quercus petraea & -0.30 & -3.0 & (Epron and Dreyer 1993) \\
Quercus petraea & -0.30 & -2.5 & (Bréda et al. 1993) \\
Quercus pubescens & -0.50 & -3.0 & (Dameisin and Rambal 1995) \\
Quercus rubra & -0.30 & -2.2 & (Crunkilton et al. 1992) \\
Quercus stellata & -0.20 & -1.5 & (Ni and Pallardy 1991) \\
Grass & Initial: mean $=-0.73$, std dev $=0.71, n=4$ \\
& Final: mean $=-2.7$, std dev $=1.2, n=4$ \\
\hline & Initial & Final & \\
Leersia hexandra & -1.7 & -3.3 & (Kirkman and Sharitz 1993) \\
Manisuris rugosa & -0.20 & -1.3 & (Kirkman and Sharitz 1993) \\
Panicum hemitomon & -0.80 & -2.3 & (Kirkman and Sharitz 1993) \\
Poa sand bergii & -0.20 & -4.0 & (Link et al. 1990) \\
Shrub & Initial: mean $=-0.81$, std dev $=0.27, n=10$ \\
& Final: mean $=-4.2$, std dev $=1.6, n=10$ \\
\hline & Initial & Final & \\
Artemesia tridentata & -0.50 & -5.0 & (DeLucia et al. 1988) \\
Artemesia tridentata & -0.80 & -4.0 & (S. D. Smith et al. 1997) \\
Artemesia tridentata & -1.0 & -3.5 & (S. D. Smith et al. 1997) \\
Ceanothus thyrsiflorus & -0.30 & -2.5 & (Tenhunen et al. 1994) \\
Colegyne ramosissima & -1.0 & -7.0 & (Smith et al. 1995) \\
Ephedra nevadensis & -1.0 & -5.0 & (Smith et al. 1995) \\
Eucalyptus socialis & -1.0 & -3.0 & (Collatz et al. 1976) \\
Haplopappus cooperi & -1.0 & -6.0 & (Smith et al. 1995) \\
Larrea tridentata & -1.0 & -4.5 & (Franco et al. 1994) \\
Nerium oleander & -0.50 & -1.8 & (Gollan et al. 1985) \\
\hline
\end{tabular}

\section{A.6.5 Vapor pressure deficit at initial and final reduction to stomatal conductance}

High leaf to atmosphere VPD is commonly observed to cause reductions in stomatal conductance. The precise mechanism, probably either a stomatal response to transpiration-induced reduction in guard cell water potential (feedback response) or a direct stomatal sensitivity to increased VPD independent of leaf water status (feedforward response), is not completely understood. Evidence exists for both the feedforward (Schulze et al. 1972) and feedback (Monteith 1995) mechanisms with some authors finding intermediate response mechanisms (Franks et al. 1997).

We collected VPD versus $g$ data for the five functional types. To do so, we extracted two values from the literature: the VPD at initial stomatal closure $\left(V_{P D}\right)$ and the VPD at final stomatal closure $\left(\mathrm{VPD}_{\mathrm{f}}\right)$. As for $\Psi$, these data are usually presented in a scatterplot format. Therefore, parameter extraction was again subjective. We assumed that responses were linear (Körner 1995) and extrapolated from the presented data to a value of zero $g$. In cases where multiple response functions were shown, we used data in which other conditions (radiation, temperature, soil water, etc.) were least limiting and for the initial exposure to drought. 
Earth Interactions - Volume 4 (2000) - Paper No. 3 - Page 54

\begin{tabular}{|c|c|c|c|}
\hline \multirow[t]{2}{*}{ ENF } & \multicolumn{3}{|c|}{$\begin{array}{l}\text { Initial: } \text { mean }=0.61, \text { std dev }=0.17, n=10 \\
\text { Final: } \text { mean }=3.1, \text { std } \operatorname{dev}=1.4, n=10\end{array}$} \\
\hline & Initial & Final & \\
\hline Abies alba & 0.50 & 2.0 & (Gueh1 1991) \\
\hline Abies cephalonica & 0.50 & 2.0 & (Guehl 1991) \\
\hline Abies marocana & 0.50 & 2.0 & (Guehl 1991) \\
\hline Abies nordmanniana & 0.50 & 2.0 & (Guehl 1991) \\
\hline Juniperus occidentalis & 1.0 & 5.0 & (Miller et al. 1993) \\
\hline Picea glauca & 0.50 & 2.5 & (Goldstein et al. 1985) \\
\hline Picea mariana & 0.50 & 6.0 & (Dang et al. 1997) \\
\hline Pinus banksiana & 0.80 & 3.8 & (Dang et al. 1997) \\
\hline Pinus sylvestris & 0.60 & 2.5 & (Kellomäki and Wang 1997) \\
\hline Pseudotsuga menziesii & 0.70 & 3.5 & (Meinzer 1982) \\
\hline DNF & 0.61 & 3.1 & Set to ENF \\
\hline \multirow[t]{2}{*}{ DBF } & \multicolumn{3}{|c|}{$\begin{array}{l}\text { Initial: } \text { mean }=1.1, \text { std dev }=0.53, n=7 \\
\text { Final: mean }=3.6, \text { std dev }=0.80, n=7\end{array}$} \\
\hline & Initial & Final & \\
\hline Acer saccharum & 1.2 & 3.4 & (Ellsworth and Reich 1992b) \\
\hline Betula pendula & 1.0 & 4.0 & (Osonubi and Davies 1980) \\
\hline Fagus sylvatica & 0.60 & 3.0 & (Kersteins 1995) \\
\hline Populus angustifolia & 2.0 & 4.7 & (Foster and Smith 1991) \\
\hline Populus nigra & 0.50 & 3.5 & (Appleby and Davies 1983) \\
\hline Populus tremuloides & 1.0 & 4.2 & (Dang et al. 1997) \\
\hline Ulmus glabra & 1.6 & 2.3 & (Appleby and Davies 1983) \\
\hline \multirow[t]{2}{*}{ Grass } & \multicolumn{3}{|c|}{$\begin{array}{l}\text { Initial: } \text { mean }=1.0, \text { std dev }=0.25, n=11 \\
\text { Final: } \text { mean }=5.0, \text { std dev }=2.7, n=11\end{array}$} \\
\hline & Initial & Final & \\
\hline Abutilon theophrasti & 1.0 & 2.0 & (Bunce 1996) \\
\hline Amaranthus hypochondriacus & 0.70 & 3.0 & (Bunce 1993) \\
\hline Ambrosia chamissonis & 0.80 & 3.0 & (Mooney and Chu 1983) \\
\hline Eriogonum latifolium & 1.0 & 3.5 & (Mooney and Chu 1983) \\
\hline Fragaria chiloensis & 1.0 & 3.5 & (Mooney and Chu 1983) \\
\hline Heteropogon contortis & 1.5 & 6.0 & (Williams and Black 1994) \\
\hline Hyparrhenia rufa & 1.0 & 5.5 & (Baruch et al. 1985) \\
\hline Leymus cinereus & 1.2 & 5.0 & (S. D. Smith et al. 1997) \\
\hline Melinis minutifolia & 1.0 & 5.5 & (Baruch et al. 1985) \\
\hline Pennisetum setaceum & 1.5 & 12 & (Williams and Black 1994) \\
\hline Trachypogon plumosus & 1.0 & 6.0 & (Baruch et al. 1985) \\
\hline \multirow[t]{2}{*}{ Shrub } & \multicolumn{3}{|c|}{$\begin{array}{l}\text { Initial: } \text { mean }=0.97, \text { std dev }=0.24, n=9 \\
\text { Final: } \text { mean }=4.1, \text { std } \operatorname{dev}=1.0, n=9\end{array}$} \\
\hline & Initial & Final & \\
\hline Arbutus unedo & 1.0 & 4.0 & (Tenhunen et al. 1982) \\
\hline Arbutus unedo & 1.0 & 3.5 & (Turner et al. 1985) \\
\hline Artemesia tridentata & 0.80 & 5.0 & (S. D. Smith et al. 1997) \\
\hline Artemesia tridentata & 0.80 & 6.0 & (S. D. Smith et al. 1997) \\
\hline Larrea tridentata & 1.0 & 3.3 & (Franco et al. 1994) \\
\hline Nerium oleander & 1.0 & 5.0 & (Gollan et al. 1985) \\
\hline Nerium oleander & 1.5 & 4.0 & (Turner et al. 1985) \\
\hline Psychotria horizontalis & 0.60 & 2.5 & (Hogan et al. 1994) \\
\hline Yucca glauca & 1.0 & 4.0 & (Roessler and Monson 1985) \\
\hline
\end{tabular}


Earth Interactions - Volume 4 (2000) - Paper No. 3 - Page 55

\section{A.7 Miscellaneous parameters}

\section{A.7.1 Water interception coefficient}

The water interception coefficient $\left(W_{\text {int }}, 1 \mathrm{LAI}^{-1}\right.$ day $\left.^{-1}\right)$ determines the amount of precipitation intercepted by the canopy. Canopy interception in turn controls the amount of precipitation entering the soil water pool. Additionally, because BIOME-BGC assumes that canopy water must be evaporated before stomatal conductance occurs (diffusion through water is 10,000 times slower than through air), canopy water interception directly impacts transpiration. For BIOME-BGC parameterization, field studies must include measurements of leaf area index and daily canopy interception. Such work is rare and results are highly dependent on the methodology used (Crockford and Richardson 1990). Nonetheless, reported values fell within a relatively narrow range. We were unable to locate any studies providing the required information for grass canopies and based on a generally erectophile leaf orientation, we set grass $W_{\text {int }}$ to $50 \%$ of the forest value.

\begin{tabular}{llc}
\hline ENF, DNF, DBF, and shrub & \multicolumn{2}{l}{ Mean $=0.045$, std dev $=0.012, n=5$} \\
\hline Pinus radiata & 0.036 & (Kelliher et al. 1992) \\
Pinus & 0.052 & (Gash et al. 1995) \\
Temperate broadleaf & 0.035 & (Klaassen et al. 1996) \\
Temperate broadleaf & 0.040 & (Lankreijer et al. 1993) \\
Tropical broadleaf & 0.063 & (Scatena 1990) \\
Grass & \multicolumn{2}{l}{ Set to 50\% of above mean } \\
\hline
\end{tabular}

\section{A.7.2 Light extinction coefficient}

The canopy light extinction coefficient $(k$, defined as the mean projection of the unit foliage area on the plane normal to incident radiation, unitless) controls canopy photosynthetically active radiation absorption. Measurements of $k$ are abundant and most are based on the adoption of Beer's law in Monsi et al. (Monsi et al. 1953): $k=\left\{-\ln \left(I_{\mathrm{i}}, I_{\mathrm{o}}\right)\right\} / \mathrm{LAI}$, where $k$ is the extinction coefficient, $\mathrm{I}_{\mathrm{i}}$ is the below-canopy radiation, $I_{0}$ is the above-canopy radiation, and LAI is leaf area index. Measurements of $I_{\mathrm{i}} / I_{\mathrm{o}}$ are usually made with a radiation-measuring device such as a sunfleck ceptometer (Decagon Instruments, Pullman, WA). LAI has been measured with many techniques, including litterfall (Heilman and Fu-Guang 1994), radiation transmittance (Chen et al. 1997), sapwood allometrics (Pierce et al. 1994; Sampson and Smith 1993), and foliage biomass (Sampson and Allen 1998). Alternatively, $k$ can be calculated through physical measurement of the contact frequency (Norman and Campbell 1989) as in Groeneveld (Groeneveld 1997). The value of $k$ is known to vary with solar angle in planophile (needle leaf) canopies (Black et al. 1991) but not in canopies with random (broad leaf) foliage orientation (Chen et al. 1997). Additionally, $k$ appears to decrease with stand age as a result of changes in three-dimensional canopy structure (Brown and Parker 1994; Heilman and Fu-Guang 1994). Literature values of $k$ were therefore drawn from a strikingly diverse pool of methodologies and stand conditions. As leaf morphology, not phenology, is the primary determinant of $k$, we included evergreen broad leaf values in the DBF calculation. We report values based on 
measurements taken around solar noon or corrected to nadir values with the cosine of the solar elevation angle correction: $k=\left\{-\ln \left(I_{1} / I_{0}\right) \cos \theta\right\} / \mathrm{LAI}$, where $\theta$ is the solar elevation angle ( 0 directly overhead). The data showing all biomes at or near 0.5 support the statement in Chen et al. (Chen 1997) that 0.5 can be taken as a first approximation of $k$ for almost all types of canopies.

\begin{tabular}{|c|c|c|}
\hline ENF & Mean & $\operatorname{tg} \mathrm{dev}=.052, n=14$ \\
\hline ENF & 0.530 & (Pierce and Running 1988) \\
\hline ENF & 0.490 & (Pierce and Running 1988) \\
\hline ENF & 0.510 & (Pierce and Running 1988) \\
\hline ENF & 0.480 & (Pierce and Running 1988) \\
\hline ENF & 0.480 & (Pierce and Running 1988) \\
\hline ENF & 0.520 & (Pierce and Running 1988) \\
\hline ENF & 0.580 & (Pierce and Running 1988) \\
\hline Pinus & 0.460 & (Sinclair and Knoer 1982) \\
\hline Pinus & 0.529 & (Lindroth and Perttu 1981) \\
\hline Pinus contorta & 0.430 & (Sampson and Smith 1993) \\
\hline Pinus taeda & 0.511 & (Sampson and Allen 1998) \\
\hline Pinus taeda & 0.477 & (Sampson and Allen 1998) \\
\hline Pinus taeda & 0.641 & (Sampson and Allen 1998) \\
\hline Pseudotsuga menziesii & 0.500 & (Black et al. 1991) \\
\hline DNF & 0.51 & Set to ENF \\
\hline DBF & Mean & std dev $=0.079, n=11$ \\
\hline Castanopsis & 0.500 & (Waring and Schlesinger 1985) \\
\hline Eucalyptus globulus & 0.500 & (Gazarini et al. 1990) \\
\hline Fagus & 0.400 & (Waring and Schlesinger 1985) \\
\hline Mixed deciduous & 0.660 & (Brown and Parker 1994) \\
\hline Populus deltoides & 0.540 & (Li et al. 1997) \\
\hline Populus hybrid & 0.622 & (Heilman and Fu-Guang 1994) \\
\hline Populus hybrid & 0.473 & (Heilman and Fu-Guang 1994) \\
\hline Populus tremuloides & 0.500 & (Chen et al. 1997) \\
\hline Rain forest & 0.600 & (Waring and Schlesinger 1985) \\
\hline Theobroma cacao & 0.610 & (Miyaji et al. 1997) \\
\hline Tropical deciduous forest & 0.610 & (Maass et al. 1995) \\
\hline Grass & Mean & std dev $=0.13, n=21$ \\
\hline Cynodon dactylon & 0.620 & (Morgan and Brown 1983) \\
\hline Distichlis spicata & 0.557 & (Groeneveld 1997) \\
\hline Festuca arundinacea & 0.439 & (Sugiyama et al. 1985) \\
\hline Festuca arundinacea & 0.411 & (Sugiyama et al. 1985) \\
\hline Festuca arundinacea & 0.401 & (Sugiyama et al. 1985) \\
\hline Festuca arundinacea & 0.406 & (Sugiyama et al. 1985) \\
\hline Festuca arundinacea & 0.322 & (Sugiyama et al. 1985) \\
\hline Festuca arundinacea & 0.533 & (Sugiyama et al. 1985) \\
\hline Festuca arundinacea & 0.550 & (Sugiyama et al. 1985) \\
\hline Festuca arundinacea & 0.372 & (Sugiyama et al. 1985) \\
\hline Festuca arundinacea & 0.514 & (Sugiyama et al. 1985) \\
\hline Festuca arundinacea & 0.441 & (Sugiyama et al. 1985) \\
\hline Festuca arundinacea & 0.533 & (Sugiyama et al. 1985) \\
\hline Festuca arundinacea & 0.369 & (Sugiyama et al. 1985) \\
\hline Festuca arundinacea & 0.384 & (Sugiyama et al. 1985) \\
\hline Festuca arundinacea & 0.594 & (Sugiyama et al. 1985) \\
\hline Festuca arundinacea & 0.314 & (Sugiyama et al. 1985) \\
\hline
\end{tabular}


Earth Interactions - Volume 4 (2000) - Paper No. 3 • Page 57

\begin{tabular}{llll}
\hline Grass & Mean $=0.48$, std dev $=0.13, n=21$ & (Continued) \\
\hline Pennisetum & 0.400 & (Matsuda et al. 1991) \\
Sasa nipponica & 0.742 & (Agata and Kamata 1979) \\
Sasa nipponica & 0.778 & (Agata and Kamata 1979) \\
Sporobolus airoides & 0.490 & (Groeneveld 1997) \\
Shrub & Mean $=0.55$, std dev $=0.10, n=8$ \\
\hline Artemesia tridentata & 0.411 & (Groeneveld 1997) \\
Atriplex canescens & 0.531 & (Groeneveld 1997) \\
Atriplex confertifolia & 0.531 & (Groeneveld 1997) \\
Atriplex lentiformis & 0.528 & (Groeneveld 1997) \\
Chrysothannus nauseosus & 0.531 & (Groeneveld 1997) \\
Lysimachia vulgaris & 0.689 & (Hirose et al. 1988) \\
Lysimachia vulgaris & 0.716 & (Hirose et al. 1988) \\
Sarcobatus vermiculatus & 0.470 & (Groeneveld 1997) \\
\hline
\end{tabular}

\section{A.7.3 Percent of leaf nitrogen in Rubisco}

Rubisco, the enzyme catalyzing the binding of $\mathrm{CO}_{2}$, is probably the most abundant protein on Earth. The percent of leaf nitrogen in Rubisco (PLNR, percent) controls potential rates of carboxylation and is, therefore, a dominant control of canopy assimilation. While some data for PLNR do exist, especially for crops, there are insufficient field data with which to parameterize natural vegetation types. PLNR can be related to more commonly measured parameters through the following equation: PLNR $=\left(V c_{\max }\right.$ SLA C: $\left.N_{\text {leaf }}\right) /(\Gamma$ act $)$, where $V c_{\max }$ is the maximum rate of carboxylation ( $\mu \mathrm{mol} \mathrm{CO}_{2} \mathrm{~m}^{-2} \mathrm{~s}^{-1}$ ), SLA is the specific leaf area $\left(\mathrm{m}^{2} \mathrm{~kg}\right.$ $\mathrm{C}^{-1}$ ), $\mathrm{C}: \mathrm{N}_{\text {leaf }}$ is the leaf $\mathrm{C}: \mathrm{N}$ ratio $\left(\mathrm{kg} \mathrm{C} \mathrm{kg} \mathrm{N}^{-1}\right), \Gamma$ is the ratio of the mass of Rubisco to the mass of nitrogen in Rubisco [7.16 kg Rubisco $\mathrm{kg} \mathrm{N}$ in Rubisco ${ }^{-1}$ (Kuehn and McFadden 1969)], and act is the Rubisco activity at $25^{\circ} \mathrm{C}[60,000$ $\mu \mathrm{mol} \mathrm{CO} \mathrm{kg} \mathrm{Rubisco}^{-1} \mathrm{~s}^{-1}$ (Woodrow and Berry 1988)]. PLNR is in units of kilograms of nitrogen in Rubisco per kilograms of leaf nitrogen, or a dimensionless fraction. Wullschleger (Wullschleger 1993) summarized $V c_{\max }$ data for a wide variety of species. We calculated ENF and DBF PLNR from $V c_{\max }$ summaries in Wullschleger (Wullschleger 1993) and SLA and C: $N_{\text {leaf }}$ presented above. Wullschleger reported that measurement temperatures for ENF were generally lower than for other biomes, possible underpredicting $V c_{\max }$. We therefore adjusted ENF $V c_{\max }$ to the mean plus one $\sigma$. We calculated a mean value for grass $\mathrm{Vc}_{\max }$ from data presented in (Wullschleger 1993). Shrub $\mathrm{Vc}_{\max }$ data were limited to hot shrubs and we chose to set shrub PLNR to the ENF value. Due to its high maximum rates of photosynthesis (Gower and Richards 1990), we set DNF PLNR to the DBF value.

Calculated PLNR data appeared to be within the range reported in the literature. In annual grasses, PLNR was 0.15 for Abutilon theophrasti and 0.09 for Amaranthus retroflexus (Tissue et al. 1995). PLNR was 0.17 in a fertilized Populus hybrid [(Brendley and Pell 1998); fertilization raises Rubisco investment], 0.11 in Alocasia macrorrhiza, an Australian tropical understory species (Seeman 1989), and 0.0457 in 1-yr-old needles of Pinus radiata (Thurnbull et al. 1998). Crop values generally range from 0.15 to 0.30 (Makino et al. 1994; Makino et al. 1992). 
Earth Interactions - Volume 4 (2000) - Paper No. 3 - Page 58

Appendix B: Notation

\begin{tabular}{|c|c|c|}
\hline Symbol & Definition & Units \\
\hline$A_{\text {smax }}$ & Maximum net canopy assimilation rate & $\mu \mathrm{mol} \mathrm{CO} \mathrm{m}^{-2} \mathrm{~s}^{-1}$ \\
\hline $\mathrm{C}$ & Carbon & Unitless \\
\hline$C: N_{d w}$ & Dead wood carbon to nitrogen ratio & $\operatorname{kg~C}(\operatorname{kg~N})^{-1}$ \\
\hline$C: N_{f r}$ & Fine root carbon to nitrogen ratio & $\operatorname{kgC}(\operatorname{kg~N})^{-1}$ \\
\hline$C: N_{\text {leaf }}$ & Leaf carbon to nitrogen ratio & $\operatorname{kg~C}(\mathrm{kg} \mathrm{N})^{-1}$ \\
\hline C: $\mathrm{N}_{\text {lit }}$ & Litter carbon to nitrogen ratio & $\mathrm{kg} \mathrm{C}(\mathrm{kg} \mathrm{N})^{-1}$ \\
\hline$C: \mathrm{N}_{\mathrm{lw}}$ & Live wood carbon to nitrogen ratio & $\operatorname{kg~C~}(\operatorname{kg~N})^{-1}$ \\
\hline $\mathrm{CRC}: \mathrm{SC}$ & $\begin{array}{l}\text { New coarse root carbon to new stem carbon } \\
\text { allocation ratio }\end{array}$ & $\operatorname{kgC}(\operatorname{kg~C})^{-1}$ \\
\hline C3G & $\mathrm{C}_{3}$ grassland & Unitless \\
\hline $\mathrm{C} 4 \mathrm{G}$ & $\mathrm{C}_{4}$ grassland & Unitless \\
\hline DBF & Deciduous broad leaf forest & Unitless \\
\hline DNF & Deciduous needle leaf forest & Unitless \\
\hline $\mathrm{DW}_{\text {cel }}$ & Dead wood cellulose & $\%$ \\
\hline $\mathrm{DW}_{\text {lig }}$ & Dead wood lignin & $\%$ \\
\hline ENF & Evergreen needle leaf forest & Unitless \\
\hline FM & Fire mortality & $1 \mathrm{yr}^{-1}$ \\
\hline FRC:LC & $\begin{array}{l}\text { New fine root carbon to new leaf carbon } \\
\text { allocation ratio }\end{array}$ & $\operatorname{kgC}(\operatorname{kg~C})^{-1}$ \\
\hline $\mathrm{FR}_{\text {cel }}$ & Fine root cellulose & $\%$ \\
\hline $\mathrm{FR}_{\mathrm{lab}}$ & Fine root labile & $\%$ \\
\hline $\mathrm{FR}_{\text {lig }}$ & Fine root lignin & $\%$ \\
\hline$g$ & Conductance & $\mathrm{m} \mathrm{s}^{-1}$ \\
\hline$g_{\mathrm{bl}}$ & Boundary layer conductance & $\mathrm{m} \mathrm{s}^{-1}$ \\
\hline$g_{\text {cut }}$ & Cuticular conductance & $\mathrm{m} \mathrm{s}^{-1}$ \\
\hline$g_{\max }$ & Maximum stomatal conductance & $\mathrm{m} \mathrm{s}^{-1}$ \\
\hline$k$ & Light extinction coefficient & Unitless \\
\hline LAI & Leaf area index & $m^{2} m^{-2}$ \\
\hline $\mathrm{LAI}_{\text {all:proj }}$ & All-sided to projected LAI ratio & LAI LAI-1 $^{-1}$ \\
\hline $\mathrm{L}_{\mathrm{cel}}$ & Litter cellulose & $\%$ \\
\hline LFRT & Leaf and fine root turnover & $1 \mathrm{yr}^{-1}$ \\
\hline $\mathrm{L}_{\mathrm{lab}}$ & Litter labile & $\%$ \\
\hline $\mathrm{L}_{\text {lig }}$ & Litter lignin & $\%$ \\
\hline LWC:TWC & $\begin{array}{l}\text { New live wood carbon to new total wood } \\
\text { carbon allocation ratio }\end{array}$ & $\operatorname{kgC}(\mathrm{kg} \mathrm{C})^{-1}$ \\
\hline LWT & Live wood turnover & $1 \mathrm{yr}^{-1}$ \\
\hline $\mathrm{N}$ & Nitrogen & Unitless \\
\hline PLNR & Percent of leaf nitrogen in Rubisco & $\%$ \\
\hline Rubisco & $\begin{array}{l}\text { Ribulose-1,5-bisphosphate carboxylase/ } \\
\text { oxygenase }\end{array}$ & Unitless \\
\hline SC:LC & $\begin{array}{l}\text { New stem carbon to new leaf carbon allocation } 1 \\
\text { ratio }\end{array}$ & $\operatorname{kg~C}(\operatorname{kg~C})^{-1}$ \\
\hline SLA & Specific leaf area & $\mathrm{m}^{-2} \mathrm{~kg} \mathrm{C}$ \\
\hline SLA $_{\text {shd:sun }}$ & Shaded to sunlit SLA ratio & SLA SLA $^{-1}$ \\
\hline SOILC & Soil carbon & $\mathrm{kg} \mathrm{C} \mathrm{m}{ }^{-2}$ \\
\hline$V \mathrm{c}_{\max }$ & Maximum rate of carboxylation & $\mu \mathrm{mol} \mathrm{CO} \mathrm{m}^{-2} \mathrm{~s}^{-1}$ \\
\hline VEMAP & $\begin{array}{l}\text { Vegetation/Ecosystem Modeling and Analysis } \\
\text { Project }\end{array}$ & Unitless \\
\hline VPD & Vapor pressure deficit & $\mathrm{kPa}$ \\
\hline $\mathrm{VPD}_{\mathrm{f}}$ & VPD at final reduction of stomatal conductance & $\mathrm{kPa}$ \\
\hline
\end{tabular}


Earth Interactions - Volume 4 (2000) - Paper No. 3 - Page 59

\begin{tabular}{llll}
\hline Symbol & \multicolumn{1}{c}{ Definition } & \multicolumn{1}{c}{ Units } & (Continued) \\
\hline VPD $_{\mathrm{i}}$ & $\begin{array}{l}\text { VPD at initial reduction of stomatal } \\
\text { conductance }\end{array}$ & $\mathrm{kPa}$ \\
$W_{\text {int }}$ & Water interception coefficient & $1 \mathrm{LAI}^{-1} \mathrm{day}^{-1}$ & \\
WOODC & Wood carbon & $\mathrm{kg} \mathrm{C} \mathrm{m}^{-2}$ & \\
WPM & Whole plant mortality & $1 \mathrm{yr}^{-1}$ \\
$\Psi$ & Water potential & $\mathrm{MPa}$ \\
$\Psi_{\mathrm{f}}$ & Predawn leaf water potential at final reduction & $\mathrm{MPa}$ \\
$\Psi_{\mathrm{i}}$ & of stomatal conductance & \\
$\Psi_{1}$ & Predawn leaf water potential at initial reduction & $\mathrm{MPa}$ \\
$\Psi_{\mathrm{lpd}}$ & of stomatal conductance & \\
$\Psi_{\mathrm{s}}$ & Leaf water potential & $\mathrm{MPa}$ \\
$\delta_{\mathrm{bl}}$ & Predawn leaf water potential & $\mathrm{MPa}$ \\
\hline
\end{tabular}

\section{Appendix C: Site Locations and Climate}

Site distribution generally encompassed the climatic range of each biome (Table C1). ENF geographic distribution was widest, ranging from western to eastern forests in inland and coastal areas. DNF was concentrated in a broad longitudinal band at the northern limit of the United States. DBF was distributed throughout the eastern United States, with a coastal western site. C3G and C4G together had broad coverage of the entire north-south-central U.S. grasslands. Shrubs were concentrated in the western United States and ranged from cold to warm climates.

Table C1. Locations and climate of simulation pixels for sensitivity analysis: Tann, mean annual temperature $\left({ }^{\circ} \mathrm{C}\right)$; Prcp, mean annual precipitation ( $\mathrm{cm}$ ); VP, mean annual water vapor pressure (Pa); SW, annual average daylight shortwave radiation $\left(\mathrm{W} \mathrm{m}^{-2}\right)$.

\begin{tabular}{lcrrrrl}
\hline & Latitude & Longitude & Tann & Prcp & VP & SW \\
\hline ENF & & & & & & \\
1 & 48.75 & -120.25 & 4.1 & 86.5 & 504.8 & 340.9 \\
2 & 48.25 & -121.75 & 8.3 & 230.0 & 802.3 & 296.6 \\
3 & 47.75 & -93.25 & 3.1 & 65.7 & 607.0 & 317.4 \\
4 & 47.25 & -123.75 & 9.5 & 304.0 & 889.5 & 287.0 \\
5 & 44.75 & -118.75 & 5.8 & 59.1 & 517.6 & 371.7 \\
6 & 44.75 & -73.25 & 7.1 & 80.7 & 835.3 & 307.2 \\
7 & 37.25 & -77.25 & 14.8 & 108.4 & 1239.7 & 368.2 \\
8 & 34.25 & -107.25 & 11.5 & 28.5 & 516.7 & 509.1 \\
9 & 32.75 & -87.75 & 17.5 & 131.8 & 1449.1 & 392.0 \\
10 & 31.75 & -110.75 & 15.9 & 47.8 & 851.5 & 480.4 \\
Mean & & & 9.7 & 114.2 & 821.4 & 367.1 \\
DNF & & & & & & \\
1 & 48.75 & -113.75 & 1.9 & 153.3 & 504.8 & 332.4 \\
2 & 47.25 & -115.75 & 5.9 & 116.1 & 660.2 & 304.7 \\
3 & 46.25 & -84.75 & 4.8 & 79.9 & 679.3 & 309.8 \\
74 & 45.75 & -67.75 & 5.1 & 102.6 & 683.5 & 328.8 \\
5 & 45.25 & -118.75 & 6.7 & 63.5 & 591.6 & 348.1 \\
\hline
\end{tabular}


Earth Interactions - Volume 4 (2000) - Paper No. 3 - Page 60

Table Cl. (Continued)

\begin{tabular}{|c|c|c|c|c|c|c|}
\hline L & Latitude & Longitude & Tann & Prcp & VP & SW \\
\hline 6 & 45.25 & -106.75 & 6.7 & 39.1 & 546.7 & 382.8 \\
\hline 7 & 44.75 & -119.25 & 7.6 & 38.7 & 513.3 & 385.6 \\
\hline 8 & 44.75 & -89.25 & 6.0 & 79.9 & 753.5 & 328.2 \\
\hline 9 & 44.75 & -72.75 & 5.1 & 111.0 & 731.0 & 324.3 \\
\hline 10 & 44.25 & -109.75 & 0.5 & 73.7 & 408.8 & 382.3 \\
\hline Mean & & & 5.0 & 85.8 & 607.3 & 342.7 \\
\hline \multicolumn{7}{|l|}{ DBF } \\
\hline 1 & 43.25 & -82.75 & 8.1 & 73.0 & 822.5 & 335.8 \\
\hline 2 & 43.25 & -70.75 & 8.1 & 110.2 & 820.7 & 335.8 \\
\hline 3 & 39.25 & -121.25 & 15.3 & 95.6 & 863.2 & 392.3 \\
\hline 4 & 39.25 & -78.25 & 11.4 & 96.4 & 985.1 & 363.9 \\
\hline 5 & 37.75 & -93.25 & 13.2 & 104.8 & 1133.8 & 373.8 \\
\hline 6 & 33.75 & -80.25 & 17.5 & 114.6 & 1411.2 & 396.0 \\
\hline 7 & 32.25 & -84.25 & 18.1 & 118.6 & 1476.7 & 393.7 \\
\hline 8 & 31.75 & -87.25 & 18.1 & 138.7 & 1490.0 & 403.2 \\
\hline 9 & 30.25 & -96.25 & 19.9 & 100.7 & 1606.5 & 393.6 \\
\hline 10 & 28.25 & -82.25 & 22.1 & 135.4 & 1863.7 & 398.1 \\
\hline Mean & & & 15.2 & 108.8 & 1247.3 & 378.6 \\
\hline \multicolumn{7}{|l|}{$\mathrm{C} 3 \mathrm{G}$} \\
\hline 1 & 45.25 & -104.25 & 6.4 & 35.8 & 568.3 & 372.2 \\
\hline 2 & 43.25 & -97.75 & 8.7 & 58.0 & 819.8 & 356.3 \\
\hline 3 & 41.75 & -106.25 & 5.3 & 29.6 & 433.2 & 419.5 \\
\hline 4 & 40.25 & -107.75 & 4.6 & 48.5 & 440.4 & 446.4 \\
\hline 5 & 40.25 & -99.75 & 11.1 & 55.1 & 861.1 & 391.9 \\
\hline 6 & 39.75 & -88.75 & 11.7 & 96.4 & 1069.6 & 352.7 \\
\hline 7 & 38.25 & -96.25 & 12.9 & 89.8 & 1114.8 & 370.1 \\
\hline 8 & 36.75 & -97.75 & 15.0 & 75.9 & 1195.7 & 384.4 \\
\hline 9 & 33.75 & -103.75 & 14.3 & 38.3 & 762.2 & 469.8 \\
\hline 10 & 33.75 & -100.75 & 16.5 & 54.0 & 1071.2 & 431.9 \\
\hline Mean & & & 10.6 & 58.0 & 833.6 & 399.5 \\
\hline \multicolumn{7}{|l|}{$\mathrm{C} 4 \mathrm{G}$} \\
\hline 1 & 42.75 & -98.25 & 9.2 & 58.0 & 849.9 & 351.2 \\
\hline 2 & 38.75 & -99.25 & 12.2 & 57.7 & 936.2 & 393.9 \\
\hline 3 & 36.25 & -102.75 & 12.9 & 37.6 & 741.6 & 454.8 \\
\hline 4 & 36.25 & -100.75 & 13.8 & 49.6 & 920.4 & 429.7 \\
\hline 5 & 35.75 & -98.25 & 15.6 & 70.8 & 1215.8 & 387.9 \\
\hline 6 & 33.75 & -101.25 & 15.2 & 50.0 & 953.7 & 441.2 \\
\hline 7 & 33.25 & -102.75 & 14.8 & 43.1 & 816.0 & 473.9 \\
\hline 8 & 31.75 & -102.25 & 17.7 & 34.7 & 934.2 & 459.6 \\
\hline 9 & 29.75 & -94.25 & 20.3 & 132.5 & 1816.1 & 364.2 \\
\hline 10 & 29.25 & -100.25 & 20.2 & 52.9 & 1328.3 & 429.6 \\
\hline Mean & & & 15.2 & 58.8 & 1051.2 & 418.6 \\
\hline \multicolumn{7}{|l|}{ Shrub } \\
\hline 1 & 44.75 & -121.25 & 9.6 & 32.9 & 514.4 & 374.0 \\
\hline 2 & 44.25 & -112.25 & 4.8 & 36.1 & 477.6 & 384.8 \\
\hline 3 & 42.25 & -119.75 & 7.6 & 28.1 & 442.7 & 408.8 \\
\hline 4 & 40.25 & -118.25 & 9.6 & 22.3 & 429.1 & 450.6 \\
\hline 5 & 40.25 & -109.25 & 8.0 & 22.6 & 428.3 & 448.0 \\
\hline 6 & 39.75 & -112.75 & 9.9 & 23.4 & 513.5 & 428.1 \\
\hline 7 & 39.25 & -119.25 & 10.1 & 19.3 & 422.2 & 451.8 \\
\hline
\end{tabular}


Earth Interactions - Volume 4 (2000) - Paper No. 3 - Page 61

Table C1. (Continued)

\begin{tabular}{lclrccc}
\hline & Latitude & Longitude & Tann & Prcp & VP & SW \\
\hline 8 & 39.25 & -112.25 & 9.1 & 35.8 & 555.9 & 432.5 \\
9 & 34.75 & -119.75 & 12.6 & 54.8 & 553.6 & 482.2 \\
10 & 29.75 & -103.75 & 17.7 & 32.9 & 826.5 & 508.8 \\
Mean & & & 9.9 & 30.7 & 516.4 & 437.0 \\
\hline
\end{tabular}

\section{References}

Aber, J. D., 1997: Why don't we believe the models. Bull. Ecol. Soc. Am., 78, 232-233.

Aber, J. D., and J. M. Melillo, 1980: Litter decomposition: Measuring relative contributions of organic matter and nitrogen to forest soils. Can. J. Bot., 58, 416-421.

Aber, J. D., and J. M. Melillo, 1982: Nitrogen immobilization in decaying hardwood leaf litter as a function of initial nitrogen and lignin content. Can. J. Bot., 60, 2263-2269.

Aber, J. D., and J. M. Melillo, 1991: Terrestrial Ecosystems. Saunders College Publishing, Chicago, $429 \mathrm{pp}$.

Aber, J. D., J. M. Melillo, and C. A. McClaugherty, 1990: Predicting long-term patterns of mass loss, nitrogen dynamics, and soil organic matter formation from initial fine litter chemistry in temperate forest ecosystems. Can. J. Bot., 68, 2201-2208.

Aber, J. D., P. B. Reich, and M. L. Goulden, 1996: Extrapolating leaf $\mathrm{CO}_{2}$ exchange to the canopy: A generalized model of forest photosynthesis compared with measurements by eddy correlation. Oecologia, 106, 257-265.

Aber, J. D., S. V. Ollinger, and C. T. Driscoll, 1997: Modeling nitrogen saturation in forest ecosystems in response to land use and atmospheric deposition. Ecol. Model., 101, 61-78.

Abrams, M. D., M. E. Kubiske, and S. A. Mostoller, 1994: Relating wet and dry year ecophysiology to leaf structure in contrasting temperate tree species. Ecology, 75, 123-133.

Acherar, M., and S. Rambal, 1992: Comparative water relations of four Mediterranean oak species. Vegetatio, 99-100, 177-184.

Agata, W., and E. Kamata, 1979: Ecological characteristics and dry matter production of some native grasses in Japan: 1. Annual growth patterns of Sasa nipponica communities. J. Jpn. Soc. Grass. Sci., 25, 103-109.

Alban, D. H., and W. A. Niering, 1975: Biomass and nutrient distribution in aspen, pine and spruce stands on the same soil types in Minnesota. J. For. Res., 8, 290-299.

Allison, F. E., R. M. Murphy, and C. J. Klein, 1963: Nitrogen requirements for the decomposition of various kinds of finely ground woods in soil. Soil Sci., 96, 187-190.

Alvera, B., 1973: Estudios en bosques de coniferas del Pirineo Central. Serie A: Pinar con acebo de San Juan del Peña. I Produccion de hojarsca. Pirineos, 109, 17-29.

Alvera, B., 1981: Productivity of forest ecosystems studied during the IBP: The woodlands data set. Dynamic Properties of Forest Ecosystems, edited by D. E. Reichle, Cambridge Univ. Press, New York, p. 615.

Anderson, J. M., 1973: The breakdown and decomposition of sweet chestnut (Castanea sativa) and beech (Fagus sylvatica) leaf litter in two deciduous woodland soils. Oecologia, 12, $275-288$.

Ando, T., K. Chiba, T. Nishimura, and T. Tanimoto, 1977: Temperate fir and hemlock forests in Shikoku. Primary Productivity in Japanese Forests, edited by T. Shidei and T. Kira, University of Tokyo Press, Tokyo, 213-245.

Appleby, R. F, and W. J. Davies, 1983: A possible evaporation site in the guard cell wall and the influence of leaf structure on the humidity response by stomata of woody plants. Oecologia, 56, 30-40. 
Auclair, D., and S. Méteyer, 1980: Méthodologie de l'évaluation de la biomasse aérienne sur pied et de la production en biomasse des taillis. Acta Oecologia, 1, 357-377.

Baldocchi, D. D., R. Valentini, S. Running, W. Oechel, and R. Dahlman, 1996: Strategies for measuring and modelling carbon dioxide and water vapour fluxes over terrestrial ecosystems. Global Change Biol., 2, 159-168.

Barclay, H. J., 1998: Conversion of total leaf area to projected leaf area in lodgepole pine and Douglas-fir. Tree Physiol., 18, 185-193.

Baruch, Z., M. M. Ludlow, and R. Davis, 1985: Photosynthetic responses of native and introduced $\mathrm{C}_{4}$ grasses from Venezuelan savannas. Oecologia, 67, 388-393.

Baskerville, G. L., 1965: Dry matter production in immature balsam fir stands. For. Sci. Monogr., 9.

Baskerville, G. L., 1966: Dry matter production in immature balsam fir stands: Roots, lesser vegetation, and total stand. For. Sci., 12, 49-53.

Bauer, G., E.-D. Schulze, and M. Mund, 1997: Nutrient contents and concentrations in relation to growth of Picea abies and Fagus sylvatica along a European transect. Tree Physiol., 17, $777-786$.

Beadle, C. L., P. G. Jarvis, H. Talbot, and R. E. Neilson, 1985: Stomatal conductance and photosynthesis in a mature Scots pine (Pinus sylvestris) forest: 2 . Dependence on environmental variables of single shoots. J. Appl. Ecol., 22, 573-586.

Beets, P. N., and D. S. Pllock, 1987: Accumulation and partitioning of dry matter in Pinus radiata as related to stand-age and thinning. N. Z. J. For. Sci., 17, 246-271.

Berg, B., 1988: Dynamics of nitrogen $\left({ }^{15} \mathrm{~N}\right)$ in decomposing Scots pine (Pinus sylvestris) needle litter. Long-term decomposition in a Scots pine forest. VI. Can. J. Bot., 66, $1539-1546$.

Berg, B., and C. McClaugherty, 1989: Nitrogen and phosphorus release from decomposing litter in relation to the disappearance of lignin. Can. J. Bot., 67, 1148-1156.

Berg, B., and G. Ekhbom, 1991: Litter mass-loss rates and decomposition patterns in some needle and leaf litter types. Long-term decomposition in a Scots pine forest. VII. Can. J. Bot., 69, 1449-1456.

Berg, B., G. Ekhbom, and C. McClaugherty, 1984: Lignin and holocellulose relations during longterm decomposition of some forest litters. Long-term decomposition in a Scots pine forest. IV. Can. J. Bot., 62, 2540-2550.

Billore, S. K., 1973: Net primary production and energetics of a grassland ecosystem at Ratlam, India. Ph.D. dissertation, Vikram University, Ujjain, India. [Available from Vikram University, Ujjain-456010, India.]

Birk, E. M., and P. M. Vitousek, 1986: Nitrogen availability and nitrogen use efficiency in loblolly pine stands. Ecology, 67, 69-79.

Black, T.A., J.-M. Chen, X. Lee, and R. M. Sagar, 1991: Characteristics of shortwave and longwave irradiances under a Douglas-fir forest stand. Can. J. For. Res., 21, 10201028.

Bliss, L. C., 1977: General summary Truelove Island ecosystem. Truelove Lowland, Devon Island, Canada: A High Arctic Ecosystem. edited by L. C. Bliss, University of Alberta Press, Edmonton, Canada, 657-675.

Bocock, K. L., 1963: Changes in the amount of nitrogen in decomposing leaf litter of sessile oak (Quercus petraea). J. Ecol., 51, 555-566.

Bocock, K. L., 1964: Changes in the amount of dry matter, nitrogen, carbon and energy in decomposing woodland leaf litter in relation to the activities of the soil fauna. J. Ecol., 52, 273-284.

Bocock, K. L., O. Gilbert, C. K. Copstick, D. C. Ewinn, J. S. Ward, and M. J. Woodman, 1964: Changes in leaf litter when placed on the surface of soils with contrasting humus types. I. Losses in dry weight of oak and ash litter. J. Soil Sci., 11, 1-9. 


\section{Earth Interactions - Volume 4 (2000) - Paper No. 3 • Page 63}

Bormann, F. H., T. G. Siccama, G. E. Likens, and R. H. Whittaker, 1970: The Hubbard Brook ecosystem study: Composition and dynamics of the tree stratum. Ecol. Monogr., 40, 373388.

Box, G. E. P., W. G. Hunter, and J. S. Hunter, 1978: Statistics for Experimenters., John Wiley, New York, $653 \mathrm{pp}$.

Bray, J. R., and L. A. Dudkiewicz, 1963: The composition, biomass and productivity of two Populus forests. Bull. Torrey Bot. Club, 90, 298-308.

Bréda, N., H. Cochard, E. Dreyer, and A. Granier, 1993: Water transfer in a mature oak stand (Quercus petraea): Seasonal evolution and effects of a severe drought. Can. J. For. Res., 23, 1136-1143.

Brendley, B. W., and E. J. Pell, 1998: Ozone-induced changes in biosynthesis of Rubisco and associated compensation to stress in foliage of hybrid poplar. Tree Physiol., 18, 81-90.

Brix, H., 1998: Effects of nitrogen fertilizer source and application rates on foliar nitrogen concentration, photosynthesis, and growth of Douglas-fir. Can. J. For. Res., 11, 775-780.

Brown, M. J., and G. G. Parker, 1994: Canopy light transmittance in a chronosequence of mixeddeciduous forests. Can. J. For. Res., 24, 1694-1703.

Buchmann, N., and E.-D. Schulze, 1999: Net $\mathrm{CO}_{2}$ and $\mathrm{H}_{2} \mathrm{O}$ fluxes of terrestrial ecosystems. Global Biogeochem. Cycles, 13, 751-760.

Bunce, J. A., 1993: Effects of doubled atmospheric carbon dioxide concentration on the response of assimilation and conductance to humidity. Plant, Cell Environ., 16, 189-197.

Bunce, J. A., 1996: Does transpiration control stomatal responses to water vapour pressure deficit. Plant, Cell Environ., 19, 131-135.

Burton, A. J., K. S. Pregitzer, and D. D. Reed, 1991: Leaf area and foliar biomass relationships in northern hardwood forests located along an $800 \mathrm{~km}$ acid deposition gradient. For. Sci., 37, 1041-1059.

Cadisch, G., H. Imhof, S. Urquiaga, R. M. Boddey, and K. E. Giller, 1996: Carbon turnover $\left(\delta^{13} C\right)$ and nitrogen mineralization potential of particulate light soil organic matter after rainforest clearing. Soil Biol. Biochem., 28, 1555-1567.

Carlson, R. E., N. N. Momen, O. Arjmand, and R. H. Shaw, 1979: Leaf conductance and leaf water potential relationships for two soybean cultivars grown under controlled irrigation. Agron. J., 71, 321-325.

Chen, J. M., P. D. Blanken, T. A. Black., M. Guilbeault, and S. Chen, 1997: Radiation regime and canopy architecture in a boreal aspen forest. Agric. For. Meteorol., 86, 107-125.

Churkina, G., and S. W. Running, 1998: Contrasting climatic controls on the estimated productivity of global terrestrial biomes. Ecosystems, 1, 206-215.

Clapp, R. B., and G. M. Hornberger, 1978: Empirical equations for some soil hydraulic properties. Water Resour. Res., 14, 601-604.

Clark, H., P. C. D. Newton, C. C. Bell, and E. M. Glasgow, 1997: Dry matter yield, leaf growth and population dynamics in Lolium perenne / Trifolium repens-dominated pasture turves exposed to two levels of elevated $\mathrm{CO}_{2}$. J. Appl. Ecol., 34, 304-316.

Clermont, L. P., and H. Schwartz, 1951: The chemical composition of Canadian woods. Pulp Pap. Mag. Can., 52, 103-105.

Cole, D. W., S. P. Gessel, and S. F. Dice, 1968: Distribution and cycling of nitrogen, phosphorus, potassium, and calcium in a second-growth Douglas-fir ecosystem. Primary Productivity and Mineral Cycling in Natural Ecosystems, edited by H. E. Young, University of Maine, Orono, ME, 197-232.

Cole, D. W., S. P. Gessel, and S. F. Dice, 1981: Productivity of forest ecosystems studied during the IBP: The woodlands data set. Dynamic Properties of Forest Ecosystems, edited by D. E. Reichle, Cambridge Univ. Press, New York, 567-672.

Collatz, J., P. J. Ferrar, and R. O. Slatyer, 1976: Effects of water stress and differential hardening 
treatments on photosynthetic characteristics of a xeromorphic shrub, Eucalyptus socialis, F. Muell. Oecologia, 23, 95-105.

Collins, N. J., J. H. Baker, and P. J. Tilbrook, 1975: Signy Island, maritime Antarctic. Structure and Function of Tundra Ecosystems, edited by T. Rosswall and O. W. Heal, Swedish Natural Science Research Council, Stockholm, Sweden, 345-374.

Comeau, P. G., and J. P. Kimmins, 1989: Above- and below-ground biomass and production of lodgepole pine on sites with differing soil moisture regimes. Can. J. For. Res., 19, 447454.

Coops, N., 1999: Improvement in predicting stand growth of Pinus radiata (D. Don) across landscapes using NOAA AVHRR and Landsat MSS imagery combined with a forest growth process model (3-PGs). Photogram. Eng. Remote Sens., 65, 1149-1156.

Cortez, J., J. M. Demard, P. Bottner, and L. J. Monrozier, 1996: Decomposition of Mediterranean leaf litters: A microcosm experiment investigating relationships between decomposition rates and litter quality. Soil Biol. Biochem., 28, 443-452.

Côte, W. A., 1977: Wood ultrastructure in relation to chemical composition. Recent Adv. Phytochem., 11, 1-14.

Coupland, R. T., and G. M. van Dyne, 1979: Natural temperate grasslands: Systems synthesis. Grassland Ecosystems of the World, edited by R. T. Coupland, Cambridge Univ. Press, New York, 97-106.

Cregg, B. M., 1994: Carbon allocation, gas exchange, and needle morphology of Pinus ponderosa genotypes known to differ in growth and survival under imposed drought. Tree Physiol., 14, 883-898.

Crockford, R. H., and D. P. Richardson, 1990: Partitioning of rainfall in a Eucalypt forest and pine plantations in southeastern Australia: I. Throughfall measurement in Eucalypt forest: Effect of method and species composition. Hydrol. Process., 4, 131-144.

Crone, E. E., and C. G. Jones, 1999: The dynamics of carbon-nutrient balance: Effects of cottonwood acclimation to short- and long-term shade on beetle feeding preferences. J. Chem. Ecol., 25, 635-656.

Crow, T. R., 1978: Biomass and production in three contiguous forests in northwest Wisconsin. Ecology, 59, 265-273.

Crunkilton, D. D., S. G. Pallardy, and H. E. Garrett, 1992: Water relations and gas exchange of northern red oak seedlings planted in a central Missouri clearcut and shelterwood. For. Ecol. Manage., 53, 117-129.

Dameisin, C., and S. Rambal, 1995: Field study of leaf photosynthetic performance by a Mediterranean deciduous oak tree (Quercus pubescens) during a severe drought. New Phytol., 131, 159-167.

Damman, A. W. H., 1964: Some forest types of central Newfoundland and their relation to environmental factors. For. Sci. Monogr., 8, 1-62.

Damman, A. W. H., 1971: Effect of vegetation changes on the fertility of a Newfoundland forest site. Ecol. Monogr., 41, 253-270.

Dang, Q.-L., H. A. Margolis, M. R. Coyea, M. Sy, and G. J. Collatz, 1997: Regulation of branchlevel gas exchange of boreal trees: Roles of shoot water potential and vapor pressure difference. Tree Physiol., 17, 521-535.

Dawson, J. O., and D. T. Funk, 1981: Seasonal change in foliar nitrogen concentration of Alnus glutinosa. For. Sci., 27, 239-243.

Day, F. P., and C. D. Monk, 1974: Vegetation patterns on a southern Appalachian watershed. Ecology, 55, 1064-1074.

Day, F. P., and C. D. Monk, 1977a: Net primary production and phenology on a southern Appalachian watershed. Am. J. Bot., 64, 1117-1125.

Day, F. P., and C. D. Monk, 1977b: Seasonal nutrient dynamics in the vegetation on a southern Appalachian watershed. Am. J. Bot., 64, 1126-1139. 


\section{Earth Interactions - Volume 4 (2000) - Paper No. 3 • Page 65}

DeAngelis, D. L., R. H. Gardiner, and H. H. Shugart Jr., 1981: Productivity of forest ecosystems studied during IBP: The woodlands data set. Dynamic Properties of Forest Ecosystems, edited by D. E. Reichle, Cambridge Univ. Press, New York, 567-672.

Deblonde, G., M. Penner, and A. Royer, 1994: Measuring leaf area index with the LI-COR LAI2000 in pine stands. Ecology, 75, 1507-1511.

Decei, I., 1981: Biomass of high productivity trees and young beech stands (Fagus sylvatica L.). Proc. Conf. on Mensuration, Growth and Yield, Kyoto, Japan, IUFRO Working Group on Forest Biomass Studies, School of Forestry and Natural Resources, University of Maine, Orono, ME, 125-128.

DeLucia, E. H., and W. H. Schlesinger, 1990: Ecophysiology of Great Basin and Sierra Nevada vegetation on contrasting soils. Plant Biology of the Basin and Range, edited by C. B. Osmond, L. F. Pitelka, and G. M. Hidy, Springer-Verlag, New York, 143-178.

DeLucia, E. H., W. H. Schlesinger, and W. D. Billings, 1988: Water relations and the maintenance of Sierran conifers on hydrothermally altered rock. Ecology, 69, 303-311.

Dentener, F. J., and P. J. Crutzen, 1994: A three-dimensional model of the global ammonia cycle. J. Atmos. Chem., 19, 331-369.

de Pury, D. G. G., and G. D. Farquhar, 1997: Simple scaling of photosynthesis from leaves to canopies without the errors of big-leaf models. Plant, Cell Environ., 20, 537-557.

Dice, S. F., 1970: The biomass and nutrient flux in a second growth douglas-fir ecosystem. Ph.D. dissertation, Thesis 18351, University of Washington, Seattle, WA, $165 \mathrm{pp}$. [Available from University of Washington, 60 Bloedel Hall, Box 352112, Seattle, WA 98195-2112.]

Donita, N., C. Bindiu, and V. Mocanu, 1981: Productivity of forest ecosystems studied during the IBP: The woodlands data set. Dynamic Properties of Forest Ecosystems, edited by D. E. Reichle, Cambridge Univ. Press, New York, 611-612.

Drew, A. P., and S. W. Running, 1975: Comparison of two techniques for measuring surface area of conifer needles. For. Sci., 22, 231-232.

Droste zu Hülshoff, B. v., 1970: Struktur, biomasse und zuwachs eines älteren fichtenbestandes. Forstwiss. ZentBl., 89, 162-171.

Duvigneaud, P., and A. Froment, 1969: Recherches sur l'ecosystème forêt. Serie E. Forêts de haute Belgique. Contribution No. 5. Eléments biogènes de l'édaphotope et phytocénose forestière. Bull. Inst. Sci. Nat. Belg., 45, 1-48.

Duvigneaud, P., and P. Kestemont, Eds., 1977: Productivité biologique en Belgique., Editions Duculot, Paris, 617.

Duvigneaud, P., P. Kestemont, and P. Ambroes, 1971: Productivité primaire des forêts tempérés d'essences feuillues caducifolices en Europe occidentale. Productivity of Forest Ecosystems, edited by P. Duvigneaud, UNESCO, Paris, 259-270.

Eastman, P. A. K., and E. L. Camm, 1995: Regulation of photosynthesis in interior spruce during water stress. Tree Physiol., 15, 229-235.

Edmonds, R. L., 1980: Litter decomposition and nutrient release in Douglas-fir, red alder, western hemlock, and Pacific silver fir ecosystems in western Washington. Can. J. For. Res., 10, 327-337.

Edmonds, R. L., 1984: Long-term decomposition and nutrient dynamics in Pacific silver fir needles in western Washington. Can. J. For. Res., 14, 395-400.

Edmonds, R. L., 1987: Decomposition rates and nutrient dynamics in small-diameter woody litter in four forest ecosystems in Washington, U.S.A. Can. J. For. Res., 17, 499-509.

Ellenberg, H., 1971: Integrated Experimental Ecology. Methods and Results of Ecosystem Research in the German Solling Project. Springer-Verlag, New York, 214 pp.

Ellenberg, H., 1981a: Productivity of forest ecosystems studied during the IBP: The woodlands data set. Dynamic Properties of Forest Ecosystems, edited by D. E. Reichle, Cambridge Univ. Press, New York 666-668. 


\section{Earth Interactions - Volume 4 (2000) - Paper No. 3 • Page 66}

Ellenberg, H., 1981b: Productivity of forest ecosystems studied during the IBP: The woodlands data set. Dynamic Properties of Forest Ecosystems, edited by D. E. Reichle, Cambridge Univ. Press, New York, 669-671.

Ellenberg, H., R. Mayer, and J. Schauermann, Eds., 1986: Ökosystemforschung Ergebnisse des Sollingprojekts 1966-1986. Eugen Ulmer Verlag, Stuttgart, Germany, 507 pp.

Ellsworth, D. S., and P. B. Reich, 1992a: Leaf mass per area, nitrogen content and photosynthetic carbon gain in Acer saccharum seedlings in contrasting forest light environments. Funct. Ecol., 6, 423-435.

Ellsworth, D. S., and P. B. Reich, 1992b: Water relations and gas exchange of Acer saccharum seedlings in contrasting natural light and water regimes. Tree Physiol., 10, 1-20.

Ellsworth, D. S., and P. B. Reich, 1993: Canopy structure and vertical patterns of photosynthesis and related leaf traits in a deciduous forest. Oecologia, 96, 169-178.

Epron, D., and E. Dreyer, 1993: Photosynthesis of oak leaves under water stress: Maintenance of high photochemical efficiency of photosystem II and occurrence of non-uniform $\mathrm{CO}_{2}$ assimilation. Tree Physiol., 13, 107-117.

Fahey, T. J., J. W. Hughes, M. Pu, and M. A. Arthur, 1978: Root decomposition and nutrient flux following whole-tree harvest of northern hardwood forest. For. Sci., 34, 744-768.

Fahey, T. J., J. B. Yavitt, and J. A. Pearson, 1985: The nitrogen cycle in lodgepole pine forests, southeastern Wyoming. Biogeochemistry, 1, 257-275.

Farquhar, G. D., S. von Caemmerer, and J. A. Berry, 1980: A biochemical model of photosynthetic $\mathrm{CO}_{2}$ assimilation in leaves of $\mathrm{C}_{3}$ species. Planta, 149, 78-90.

Fassnacht, K. S., S. T. Gower, J. M. Norman, and R. E. McMurtie, 1994: A comparison of optical and direct methods for estimating foliage surface area index in forests. Agric. For. Meteorol., 71, 183-207.

Field, C., and H. A. Mooney, 1983: Leaf age and seasonal effects on light, water and nitrogen use efficiency in a California (USA) shrub. Oecologia, 56, 348-355.

Field, C., J. Merino, and H. A. Mooney, 1983: Compromises between water-use efficiency and nitrogen-use efficiency in five species of California evergreens. Oecologia, 60, 384-389.

Fogel, R., 1983: Root turnover and productivity of coniferous forests. Plant Soil, 71, 75-85.

Ford, E. D., and P. J. Newbould, 1970: Stand structure and dry weight production through the sweet chestnut (Castanea sativa Mill.) coppice cycle. J. Ecol., 58, 275-296.

Ford, E. D., and P. J. Newbould, 1971: The leaf canopy of a coppiced deciduous woodland. $J$. Ecol., 59, 843-862.

Forrest, W. G., 1973: Biological and economic production in radiata pine plantations. J. Appl. Ecol., 10, 259-267.

Forrest, W. G., and J. D. Ovington, 1970: Organic matter changes in an age series of Pinus radiata plantations. J. Appl. Ecol., 7, 177-186.

Foster, J. R., and W. K. Smith, 1991: Stomatal conductance patterns and environment in high elevation phreatophytes of Wyoming. Can. J. Bot., 69, 647-655.

Franco, A. C., A. G. de Soyza, R. A. Virginia, J. F. Reynolds, and W. G. Whitford, 1994: Effects of plant size and water relations on gas exchange and growth of the desert shrub Larrea tridentata. Oecologia, 97, 171-178.

Franks, P. J., I. R. Cowan, and G. D. Farquhar, 1997: The apparent feedforward response of stomata to air vapour pressure deficit: Information revealed by different experimental procedures with two rainforest trees. Plant, Cell Environ., 20, 142-145.

Fujimori, T., 1971: Primary production of a young Tsuga heterophylla stand and some speculation about biomass of forest communities on the Oregon coast. Rep. PNW-123, USDA Forest Service, Portland, OR, 1-11.

Fujimori, T., S. Kawanabe, H. Saito, C. C. Grier, and T. Shidei, 1976: Biomass and primary production in forests of three major vegetation zones of the north-western United States. $J$. Jpn. For. Soc., 58, 360-373. 
Fyles, J. W., and W. B. McGill, 1987: Decomposition of boreal forest litters from central Alberta under laboratory conditions. Can. J. For. Res., 17, 109-114.

Gallardo, A., and J. Merino, 1993: Leaf decomposition in two Mediterranean ecosystems of southwest Spain: Influence of substrate quality. Ecology, 74, 152-161.

Gallego, H. A., M. Rico, G. Moreno, and I. Santa Regina, 1994: Leaf water potential and stomatal conductance in Quercus pyrenaica wild forests: Vertical gradients and response to environmental factors. Tree Physiol., 14, 1039-1047.

Gandar, M. V., 1982: Tropic ecology and plant/herbivore energetics. Ecology of Tropical Savannas, edited by W. D. Billings et al., Springer-Verlag, New York, 514-534.

Garnier, E., P. Cordonnier, J.-L. Guillerm, and L. Soniér, 1997: Specific leaf area and nitrogen concentration in annual and perennial grass species growing in Mediterranean old-fields. Oecologia, 11, 490-498.

Gash, J. H. C., C. R. Lloyd, and G. Lachaud, 1995: Estimating sparse forest rainfall interception with an analytical model. J. Hydrol., 170, 79-86.

Gausman, H. W., J. H. Everitt, and D. E. Escobar, 1979: Seasonal nitrogen concentration and reflectance of seven woody plant species. J. Rio Grande Valley Hort. Soc., 33, 101-104.

Gazarini, L. C., M. C. C. Araujo, N. Borralho, and J. S. Pereira, 1990: Plant area index in Eucalyptus globulus plantations determined indirectly by a light interception method. Tree Physiol., 7, 107-113.

Geiger, D. R., and J. C. Servaites, 1994: Diurnal regulation of photosynthetic carbon metabolism in $\mathrm{C}_{3}$ plants. Annu. Rev. Plant Physiol. Plant Mol. Biol., 45, 235-256.

Gholz, H. L., 1982.: Environmental limits on aboveground net primary production. Leaf area, and biomass in vegetation zones of the Pacific Northwest. Ecology, 63, 469-481.

Gholz, H. L., F. Fitz, and R. H. Waring, 1976: Leaf area differences associated with old-growth forest communities in the western Oregon Cascades. Can. J. For. Res., 6, 49-57.

Gholz, H. L., C. C. Grier, A. G. Campbell, and A. T. Brown, 1979: Equations for estimating biomass and leaf area of plants in the Pacific Northwest. For. Res. Lab. Rep. 41, Oregon State University, Corvallis, OR, 39.

Gholz, H. L., C. S. Perry, W. P. Cropper Jr., and L. C. Hendry, 1985: Litterfall, decomposition, and nitrogen and phosphorous dynamics in a chronosequence of slash pine (Pinus elliottii) plantations. For. Sci., 31, 463-478.

Gholz, H. L., L. C. Hendry, and W. P. J. Croppper, 1986: Organic matter dynamics of fine roots in plantations of slash pine (Pinus elliottii) in north Florida. Can. J. For. Res., 16, 529538.

Gilbert, O., and K. L. Bocock, 1960: Changes in leaf litter when placed on the surface of soils with contrasting humus types. II. Changes in the nitrogen content of oak and ash litter. $J$. Soil Sci., 11, 10-13.

Glassy, J. M., and S. W. Running, 1994: Validating diurnal climatology logic of the MT-CLIM model across a climatic gradient in Oregon. Ecol. Appl., 4, 248-257.

Goldstein, G. H., L. B. Brubaker, and T. M. Hinckley, 1985: Water relations of white spruce (Picea glauca (Moench) Voss) at tree line in north central Alaska. Can. J. For. Res., 15, 1080-1087.

Gollan, T., N. C. Turner, and E.-D. Schulze, 1985: The responses of stomata and leaf gas exchange to vapor pressure deficits and soil water content III. In the sclerophyllous woody species Nerium oleander. Oecologia, 65, 356-362.

Gordon, A. G., 1981: Productivity of forest ecosystems studied during the IBP: The woodlands data set. Dynamic Properties of Forest Ecosystems, edited by D. E. Reichle, Cambridge Univ. Press, New York, 576-579.

Gosz, J. R., 1980: Biomass distribution and production budget for a non-aggrading forest ecosystem. Ecology, 61, 507-514. 


\section{Earth Interactions - Volume 4 (2000) - Paper No. 3 - Page 68}

Gosz, J. R., G. E. Likens, and F. H. Bormann, 1972: Nutrient content of litterfall in the Hubbard Brook Experimental Forest, New Hampshire. Ecology, 53, 769-784.

Gosz, J. R., G. E. Likens, and F. H. Bormann, 1973: Nutrient release from decomposing leaf and branch litter in the Hubbard Brook Forest, New Hampshire. Ecol. Monogr., 43, 173-191.

Goulden, M. L., J. W. Munger, S.-M. Fan, B. C. Daube, and S. C. Wofsy, 1996: Exchange of carbon dioxide by a deciduous forest: Response to interannual climate variability. Science, 271, 1576-1578.

Gower, S. T., 1987: A comparison of above-ground productivity and carbon and nutrient allocation patterns of a deciduous (Larix occidentalis) and an evergreen (Pinus contorta) conifer in the east slopes of the Washington Cascades. Ph.D. dissertation, Thesis 34825 , University of Washington, Seattle, WA, $140 \mathrm{pp}$. [Available from University of Washington, 60 Bloedel Hall, Box 352112, Seattle, WA 98195-2112.]

Gower, S. T., and J. H. Richards, 1990: Larches: Deciduous conifers in an evergreen world. Bioscience, 40, 818-826.

Gower, S. T., and Y. Son, 1992: Difference in soil and leaf litterfall nitrogen dynamics for five forest plantations. Soil Sci. Soc. Am. J., 56, 1959-1966.

Gower, S. T., C. C. Grier, D. J. Vogt, and K. A. Vogt, 1987: Allometric relations of deciduous (Larix occidentalis) and evergreen conifers (Pinus contorta and Pseudotsuga menziesii) of the Cascade Mountains in central Washington. Can. J. For. Res., 17, 630-634.

Gower, S. T., K. A. Vogt, and C. C. Grier, 1992: Carbon dynamics of Rocky Mountain Douglasfir: Influence of water nutrient availability. Ecol. Monogr., 62, 43-65.

Grier, C. C., 1976: Biomass, productivity and nitrogen-phosphorous cycles in hemlock-spruce stands of the central Oregon coast. Proceedings of the Western Hemlock Management Conference W. A. Atkinson and R. J. Zasoski, Eds., College of Forest Resources, University of Washington, Seattle, WA, 71-87.

Grier, C. C., 1978: A Tsuga heterophylla-Picea sitchensis ecosystem of coastal Oregon: Decomposition and nutrient balances of fallen logs. Can. J. For. Res., 8, 198-206.

Grier, C. C., and W. A. Milne, 1981: Regression equation for calculating component biomass of young Abies amabilis (Dougl.) Forbes. Can. J. For. Res., 11, 184-187.

Grier, C. C., D. W. Cole, C. T. Dyrness, and R. L. Fredriksen, 1974: Nutrient cycling in 37- and 450-year-old Douglas fir ecosystems. Integrated Research in the Coniferous Forest Biome University of Washington, Seattle, WA, 21-36.

Grier, C. C., K. A. Vogt, M. R. Keyes, and R. L. Edmonds, 1981: Biomass distribution and aboveand below-ground production in young and mature Abies amabilis zone ecosystems of the Washington Cascades. Can. J. For. Res., 11, 155-167.

Groeneveld, D. P., 1997: Vertical point quadrant sampling and an extinction factor to calculate leaf area index. J. Arid Environ., 36, 475-485.

Guehl, J. M., 1991: Sensitivity of leaf gas exchange to atmospheric drought, soil drought, and water-use efficiency in some Mediterranean Abies species. Can. J. For. Res., 21, 15071515.

Hacke, U., and J. J. Sauter, 1995: Vulnerability of xylem to embolism in relation to leaf water potential and stomatal conductance in Fagus sylvatica f. and Populus balsamifera. J. Exp. Bot., 46, 1177-1183.

Hanley, D. P., 1976: Tree biomass and productivity estimated for three habit types of northern Idaho. College of Forestry Rep. 14, University of Idaho, Moscow, ID, 15 pp.

Harmon, M. E., J. F. Franklin, F. J. Swanson, P. Sollins, S. V. Gregory, J. D. Lattin, N. H. Anderson, G.W. Cline, N. G. Aumen, J. R. Sedell, G. W. Lienkaemper, K. Cromack Jr., and K. W. Cummins, 1986: Ecology of coarse woody debris in temperate ecosystems. Adv. Ecol. Res., 15, 133-302.

Harris, W. F, and G. S. Henderson, 1981: Productivity of forest ecosystems studied during the 
IBP: The woodlands data set. Dynamic Properties of Forest Ecosystems, edited by D. E. Reichle, Cambridge Univ. Press, New York, 658-661.

Harris, W. F., R. A. Goldstein, and G. S. Henderson, 1973: Analysis of forest biomass pools, annual primary production and turnover of biomass for a mixed deciduous forest watershed. IUFRO Biomass Studies, edited by H. E. Young, University of Maine, Orono, ME, 43-64.

Harris, W. F., R. S. Kinerson, and N. T. Edwards, 1977: Comparison of belowground biomass of natural deciduous forest and loblolly pine plantations. Pedobiologia, 17, 369-381.

Hart, S. C., M. K. Firestone, and E. A. Paul, 1992: Decomposition and nutrient dynamics of ponderosa pine needles in a Mediterranean-type climate. Can. J. For. Res., 22, 306-314.

Hatiya, K., K. Doi, and R. Kobayashi, 1965: Analysis of the growth of Japanese red pine (Pinus densiflora) stands. Bull. Gov. For. Exp. Stn. Tokyo, 176, 75-88.

Hayes, D. C., and T. R. Seastedt, 1987: Root dynamics of tallgrass prairie in wet and dry years. Can. J. Bot., 65, 787-791.

Heilman, P. E., and X. Fu-Guang, 1994: Effects of nitrogen fertilization on leaf area, light interception, and productivity of short-rotation Populus trichocarpa $\mathrm{x}$ Populus deltoides hybrids. Can. J. For. Res., 24, 166-173.

Henderson-Sellers, B., and A. Henderson-Sellers, 1996: Sensitivity evaluation of environmental models using fractional factorial analysis. Ecol. Model., 86, 291-295.

Hirose, T., M. J. A. Werger, T. L. Pons, and J. W. A. van Rheenen, 1988: Canopy structure and leaf nitrogen distribution in a stand of Lysimachia vulgaris $\mathrm{L}$. as influenced by stand density. Oecologia, 77, 145-150.

Hogan, K. P., A. P. Smith, J. L. Araus, and A. Saavedra, 1994: Ecotypic differentiation of gas exchange responses and leaf anatomy in a tropical forest understory shrub from areas of contrasting rainfall regimes. Tree Physiol., 14, 819-831.

Holbrook, N. M., J. L. Whitbeck, and H. A. Mooney, 1995: Drought responses of neotropical dry forest trees. Seasonally Dry Tropical Forests, edited by S. H. Bullock, H. A. Mooney, and E. Medina, Cambridge Univ. Press, New York, 243-276.

Holland, E. A., B. H. Braswell, J.-F. Lamarque, A. Townsend, J. Sulzman, J.-F. Müller, F. Dentener, G. Brasseur, H. I. Levy, J. E. Penner, and G.-J. Roelofs, 1997: Variations in the predicted spatial distribution of atmospheric nitrogen deposition and their impact on carbon uptake by terrestrial ecosystems. J. Geophys. Res., 102 (D13), 15,849-15,866.

Holm, E., and V. Jensen, 1981: Productivity of forest ecosystems studied during the IBP: The woodlands data set. Dynamic Properties of Forest Ecosystems, edited by D. E. Reichle, Cambridge Univ. Press, New York, 581.

Houghton, R. A., E. A. Davidson, and G. M. Woodwell, 1998: Missing sinks, feedbacks, and understanding the role of terrestrial ecosystems in the global carbon balance. Global Biogeochem. Cycles, 12, 25-34.

Hughes, M. K., 1970: Investigations of the ecosystem energetics of an English woodland. Ph.D. dissertation, University of Durham, Durham, United Kingdom, 188 pp. [Available from Durham University Library, Stockton Road, Durham DH1 3LY, United Kingdom.]

Hughes, M. K., 1971: Tree biocontent, net production and litterfall in a deciduous woodland. Oikos, 22, 62-73.

Hunt, E. R., Jr., S. C. Piper, R. Nemani, C. D. Keeling, R. D. Otto, and S. W. Running, 1996: Global net carbon exchange and intra-annual atmospheric $\mathrm{CO}_{2}$ concentrations predicted by an ecosystem process model and three-dimensional atmospheric transport model. Global Biogeochem. Cycles, 10, 431-456.

Hunt, H. W., E. R. Ingham, D. C. Coleman, E. T. Elliott, and C. P. P. Reid, 1988: Nitrogen limitation of production and decomposition in prairie, mountain meadow, and pine forest. Ecology, 69, 1009-1016.

Hytteborn, H., 1975: Deciduous woodland at Andersby, eastern Sweden. Above-ground tree and shrub production. Acta Phytogeogr. Suec., 61. 
Igboanugo, A. B., 1996: Stomatal resistance, respiration and net photosynthesis in sun and shade leaves of Quercus petraea and Nothofagus procera seedlings. Niger. J. Bot., 9-10, 15-24.

Intergovernmental Panel on Climate Change 1995: Climate Change 1995: The Science of Climate Change. Cambridge Univ. Press, New York, 572 pp.

Jain, S. K., 1971: Production studies in some grasslands of Sagar. Ph.D. dissertation, Saugar University, Sagar, India. [Available from Saugar University, Sagar 470003, India.]

Jakus, P., 1981: Productivity of forest ecosystems studied during the IBP: The woodlands data set. Dynamic Properties of Forest Ecosystems, edited by D. E. Reichle, Cambridge Univ. Press, New York, p. 586.

Jenkin, J. F., 1975: Macquarie Island, Subantarctic. Structure and Function of Tundra Ecosystems, edited by T. Rosswall and O. W. Heal, Swedish Natural Science Research Council, Stockholm, Sweden, 375-397.

Johnson, F. L., and P. G. Risser, 1974: Biomass, annual net primary production, and dynamics of six mineral elements in a Post oak-Blackjack oak forest. Ecology, 55, 1246-1258.

Jonasson, S., H. Medrano, and J. Flexas, 1997: Variation in leaf longevity of Pistacia lentiscus and its relationship to sex and drought stress inferred from leaf $\delta^{13} \mathrm{C}$. Funct. Ecol., 11, 282 289.

Jose, S., and A. R. Gillespie, 1996: Aboveground production efficiency and canopy nutrient contents of mixed-hardwood forest communities along a moisture gradient in the central United States. Can. J. For. Res., 26, 2214-2223.

Joslin, J. D., and G. S. Henderson, 1987: Organic matter and nutrients associated with fine root turnover in a white oak stand. For. Sci., 33, 330-346.

Kakubari, Y., 1977: Beech forests in the Naeba Mountains: Distribution of primary productivity along the altitudinal gradient. Primary Productivity in Japanese Forests, edited by T. Shidei and T. Kira, University of Tokyo Press, Tokyo, 201-212.

Kaldy, J. E., and K. H. Dunton, 1999: Ontogenetic photosynthetic changes, dispersal and survival of Thalassia testudinum (turtle grass) seedlings in a sub-tropical lagoon. J. Exp. Mar. Biol. Ecol., 240, 193-212.

Kawahara, T., Y. Tadaki, I. Takeuchi, A. Sato, K. Higuchi, and K. Kamo, 1979: Productivity and cycling of organic matter in natural Fagus crenata and two planted Chamaecyparis obtusa forests. Jpn. J. Ecol., 29, 387-395.

Keeling, C. D., 1994: Global historical $\mathrm{CO}_{2}$ emissions. Trends '93: A Compendium of Data on Global Change, edited by T. A. Boden et al., Oak Ridge National Laboratory, Oak Ridge, TN, 501-504.

Kelliher, F. M., D. Whitehead, and D. S. Pollock, 1992: Rainfall interception by trees and slash in young Pinus radiata D. Don stands. J. Hydrol., 131, 187-204.

Kelliher, F. M., R. Leuning, M. R. Raupach, and E.-D. Schulze, 1995: Maximum conductances for evaporation from global vegetation types. Agric. For. Meteorol., 73, 1-16.

Kellomäki, S., and K.-Y. Wang, 1997: Effects of elevated $\mathrm{O}_{3}$ and $\mathrm{CO}_{2}$ concentrations on photosynthesis and stomatal conductance in Scots pine. Plant, Cell Environ., 20, 995-1006.

Kern, J. S., 1994: Spatial patterns of soil organic carbon in the contiguous United States. Soil Sci. Soc. Am. J., 58, 439-555.

Kern, J. S., 1995: Geographic patterns of soil water-holding capacity in the contiguous United States. Soil Sci. Soc. Am. J., 59, 1126-1133.

Kersteins, G., 1995: Cuticular water permanence of European trees and shrubs grown in polluted and unpolluted atmospheres, and its relation to stomatal response to humidity in beech (Fagus sylvatica L.). New Phytol., 129, 495-503.

Kestemont, P., 1971: Productivité primaire des taillis simples et concept de nécromasse. Productivity of Forest Ecosystems, edited by P. Duvigneaud, UNESCO, Paris, 271-279.

Kestemont, P., 1975: Biomasse, nécromasse et productivité aériennes ligneuses de quelques peu- 
plements forestiers en Belgique. Ph.D. dissertation, Free University of Brussels, Brussels, Belgium. [Available from Bibliothque des Sciences et Techniques, CP 174 30, Ave. Depage, 1050 Bruxelles, Belgium.]

Keyes, M. R., and C. C. Grier, 1981: Above- and below-ground net production in 40-year-old Douglas-fir stands on low and high productivity sites. Can. J. For. Res., 11, 599-605.

Kimball, J. S., S. W. Running, and R. Nemani, 1997a: An improved method for estimating surface humidity from daily minimum temperatures. Agric. For. Meteorol., 85, 87-98.

Kimball, J. S., P. E. Thornton, M. A. White, and S. W. Running, 1997b: Simulating forest productivity and surface-atmosphere carbon exchange in the BOREAS study region. Tree Physiol., 17, 589-599.

Kimball, J. S., M. A. White, and S. W. Running, 1997c: BIOME-BGC simulations of stand hydrologic processes for BOREAS. J. Geophys. Res., 102, 29,043-29,051.

Kimmins, J. P., and B. C. Hawkes, 1978: Distribution and chemistry of fine roots in a white spruce-subalpine fir stand in British Columbia: Implication for management. Can. J. For. Res., 8, 256-279.

Kimura, M., 1963: Dynamics of vegetation in relation to soil development in northern Yatsugataki Mountains. Jpn. J. Bot., 18, 255-287.

Kimura, M., 1969: Ecological and physiological studies on the vegetation on Mount Shimagare. VII. Analysis of production processes of young stand based on the carbohydrate economy. Bot. Mag., Tokyo, 82, 6-19.

Kimura, M., I. Mototani, and K. Hogetsu, 1968: Ecological and physiological studies on the vegetation on Mount Shimagare. VI. Growth and dry matter production of young Abies stands. Bot. Mag., Tokyo, 81, 287-296.

Kinerson, R. S., C. W. Ralston, and C. G. Wells, 1977: Carbon cycling in a loblolly pine plantation. Oecologia, 29, 1-10.

Kirkman, L. K., and R. R. Sharitz, 1993: Growth in controlled water regimes of three grasses common in freshwater wetlands of the southeastern U.S. Aquat. Bot., 44, 345-359.

Kitazawa, Y., 1981: Productivity of forest ecosystems studied during the IBP: The woodlands data set. Dynamic Properties of Forest Ecosystems, edited by D. E. Reichle, Cambridge Univ. Press, New York, p. 603.

Kittel, T. G. F., J. A. Royle, C. Daly, N. A. Rosenbloom, W. P. Gibson, H. H. Fisher, D. S. Schimel, D. S. Berliner, and VEMAP2 Participants, A gridded historical (1895-1993) bioclimate dataset for the conterminous United States. Preprints, 10th Conf. on Applied Climatology, Reno, NV, Amer. Meteor. Soc., 219-222.

Klaassen, W., H. J. M. Lankreijer, and A. W. L. Veen, 1996: Rainfall interception near a forest edge. J. Hydrol., 185, 349-361.

Kloeppel, B. D., S. T. Gower, I. W. Treichel, and S. Kharuk, 1998: Foliar carbon isotope discrimination in Larix species and sympatric evergreen conifers: A global comparison. Oecologia, 114, 153-159.

Knapp, A. K., 1985: Effect of fire and drought on the ecophysiology of Andropogon gerardii and Panicum virgatum in a tallgrass prairie. Ecology, 66, 1309-1320.

Koch, G. W., J. S. Amthor, and M. L. Goulden, 1994: Diurnal patterns of leaf photosynthesis, conductance and water potential at the top of a lowland rain forest canopy in Cameroon: Measurements from the Radeau des Cimes. Tree Physiol., 14, 347-360.

Koerper, G. J., and C. J. Richardson, 1980: Biomass and net annual primary production regressions for Populus grandidenta in northern lower Michigan. Can. J. For. Res., 92-101.

Körner, C., 1995: Leaf diffusive conductances in the major vegetation types of the globe. Ecophysiology of Photosynthesis, edited by E.-D. Schulze and M. M. Caldwell, Springer-Verlag, New York, 463-490.

Körner, C., P. Bannister, and A. F. Mark, 1986: Altitudinal variation in stomatal conductance, 


\section{Earth Interactions - Volume 4 (2000) - Paper No. 3 • Page 72}

nitrogen count, and leaf anatomy in different plant forms in New Zealand. Oecologia, 69, $577-588$.

Kozlowski, T. T., and S. G. Pallardy 1997: Physiology of Woody Plants. Academic Press, San Diego, $411 \mathrm{pp}$.

Kozlowski, T. T., P. J. Kramer, and S. G. Pallardy 1991: The Physiological Ecology of Woody Plants. Academic Press, San Diego, 657 pp.

Krumlik, G. J., and J. P. Kimmins, 1976: Biomass and nutrient distribution in two undisturbed forest ecosystems. 16th IUFRO World Congress, Oslo, Norway, IUFRO, 92-103.

Küchler, A. W., 1964: Potential natural vegetation of the conterminous United States. Manual to accompany the map. Spec. Publ. 36, Am. Geogr. Soc., New York, 143 pp.

Küchler, A. W. 1975: Potential Natural Vegetation of the United States. 2d ed., Map 1:3,168,000, Am. Geogr. Soc., New York.

Kudo, G., 1995: Leaf traits and shoot performance of an evergreen shrub, Ledum palustre ssp. decumbens, in accordance with latitudinal change. Can. J. Bot., 73, 1451-1456.

Kuehn, G. D., and B. A. McFadden, 1969: Ribulose 1,5-diphosphate carboxylase from Hydrogenomonas eutropha and Hydrogenomonas facilis. II. Molecular weight, subunits, composition, and sulfhydryl groups. Biochem. J., 8, 2403-2408.

Kull, O., and Ü. Niinemets, 1993: Variations in leaf morphometry and nitrogen concentration in Betula pendula Roth., Corylus avellana L. and Lonicera xylosteum L.. Tree Physiol., 12, 31-318.

Kull, O., A. Koppel, and A. Noormets, 1998: Seasonal changes in leaf nitrogen pools in two Salix species. Tree Physiol., 18, 45-51.

Kumar, A., and M. C. Joshi, 1972: The effect of grazing on the structure and productivity of vegetation near Pilani, Rajasthan, India. J. Ecol., 60, 665-675.

Lacey, C. J., J. Walker, and I. R. Noble, 1982: Fire in Australian tropical savannas. Ecology of Tropical Savannas, edited by W. D. Billings et al., Springer-Verlag, New York, 246-272.

Lajtha, K., and F. J. Barnes, 1991: Carbon gain and water use in pinyon pine-juniper woodlands of northern New Mexico: Field versus phytotron chamber measurements. Tree Physiol., 9, 59-67.

Lankreijer, H. J. M., M. J. Hendricks, and W. Klaassen, 1993: A comparison of models simulating rainfall interception of forests. Agric. For. Meteorol., 64, 187-199.

Lavrenko, E. M., and Z. V. Karamysheva, 1992: Steppes of the former Soviet Union. Natural Grasslands, edited by R. T. Coupland, Elsevier, New York, 3-59.

Lawson, G. J., G. Cottam, and O. L. Loucks, 1981: Productivity of forest ecosystems studied during the IBP: The woodlands data set. Dynamic Properties of Forest Ecosystems, edited by D. E. Reichle, Cambridge Univ. Press, New York, 663-664.

Lemée, G., 1978: La hêtraie naturelle de Fontainebleau. Problemes d'Ecologie: Structure et Fonctionnement des Ecosystèmes Terrestres, edited by M. Lamotte and F. Bourlière, Masson, Paris, 75-127.

Leverenz, J., J. D. Deans, E. D. Ford, P. G. Jarvis, R. Milne, and D. Whitehead, 1982: Systematic spatial variation of stomatal conductance in a Sitka (Picea sitchensis) spruce plantation. $J$. Appl. Ecol., 19, 835-852.

Lewis Smith, R. I., and D. W. H. Walton, 1975: South Georgia, Subantarctic. Structure and Function of Tundra Ecosystems, edited by T. Rosswall and O. W. Heal, Swedish Natural Science Research Council, Stockholm, Sweden, 399-423.

Li, H., Z. Wen, M. Huang, and M. Wang, 1997: A genetic study on characteristics of crown light interception in Populus deltoides. Can. J. For. Res., 27, 1465-1470.

Linder, S., and B. Axelsson, 1982: Changes in carbon uptake and allocation patterns as a result of irrigation and fertilization in a young Pinus sylvestris stand. Proc. IUFRO Workshop, Corvallis, OR, IUFRO, 38-44. 


\section{Earth Interactions - Volume 4 (2000) - Paper No. 3 • Page 73}

Lindroth, A., and D. Perttu, 1981: Simple calculation of extinction coefficient of forest stands. Agric. Meteorol., 25, 97-110.

Link, S. O., G. W. Gee, and J. L. Downs, 1990: The effect of water stress on phenological and ecophysiological characteristics of cheatgrass and Sandberg's bluegrass. J. Range Manage., 43, 506-513.

Lutz, H. J., and A. C. Cline, 1947: Results of the first thirty years of experimentation in silviculture in the Harvard Forest, 1908-1938. Part I: The conversion of stands of old field origin by various methods of cutting and subsequent cultural treatments. Harvard Univ. For. Bull., 23.

Maass, J. M., J. M. Vose, W. T. Swank, and A. Martinez-Yrizar, 1995: Seasonal changes of leaf area index (LAI) in a tropical deciduous forest in west Mexico. For. Ecol. Manage., 74, 171-180.

Madgwick, H. A. I., 1962: Studies in the growth and nutrition of Pinus resinosa Ait. Ph.D. dissertation, State University College of Forestry, Syracuse University, Syracuse, NY, 111 pages.

Madgwick, H. A. I., 1968: Seasonal changes in biomass and annual production of an old field Pinus virginiana stand. Ecology, 49, 149-152.

Madgwick, H. A. I., E. H. White, G. K. Xydias, and A. L. Leaf, 1970: Biomass of Pinus resinosa in relation to potassium nutrition. For. Sci., 16, 154-159.

Madgwick, H. A. I., D. S. Jackson, and P. J. Knight, 1977a: Above-ground dry matter, energy and nutrient contents of trees in an age series of Pinus radiata plantations. N. Z. J. For. Sci., 7, 445-468.

Madgwick, H. A. I., D. S. Jackson, and P. J. Knight, 1977b: Dry matter and nutrient data on Pinus radiata trees and stands. Soils and Site Productivity Rep. 94 (unpublished), New Zealand Forest Service, Forest Research Institute, Rotorua, New Zealand, 21 pp..

Makino, A., H. Sakashita, J. Hidema, M. T., K. Ojima, and B. Osmond, 1992: Distinctive responses of ribulose-1.5-bisphosphate carboxylase and carbonic anhydrase in wheat leaves to nitrogen nutrition and their possible relationships to $\mathrm{CO}_{2}$-transfer resistance. Plant Physiol., 100, 1737-1743.

Makino, A., H. Nakano, and T. Mae, 1994: Responses of ribulose-1, 5-bisphosphate carboxylase, cytochrome $f$, and sucrose synthesis enzymes in rice leaves to leaf nitrogen and their relationships to photosynthesis. Plant Physiol., 105, 173-179.

Mälkönen, E., 1974: Annual primary production and nutrient cycle in some Scots pine stands. Commun. Inst. For. Fenn. (Helsinki), Vol. 5, No. 84, Finnish Forest Research Institute, Helsinki, Finland, 87 pp.

Mälkönen, E., 1977: Annual primary production and nutrient cycle in a birch stand. Commun. Inst. For. Fenn (Helsinki), Vol. 5, No. 91, Finnish Forest Research Institute, Helsinki, Finland, $35 \mathrm{pp}$.

Maruyama, K., 1971: Effect of altitude on dry matter production of primeval Japanese beech forest communities in Naeba Mountains. Mem. Fac. Agric. Niigata Univ., 9, 87-171.

Maruyama, K., 1977: Beech forests in the Naeba Mountains. Comparison of forest structure biomass and net primary productivity between the upper and lower parts of beech forest zone. Primary Productivity in Japanese Forests, edited by T. Shidei and T. Kira, University of Tokyo Press, Tokyo, 186-201.

Matsuda, Y., F. Kubota, and W. Agata, 1991: Analytical study on high productivity in Napier grass (Pennisetum purpureum Schumach): 1. Comparison of the characteristics of dry matter production between Napier grass and corn plants. J. Jpn. Soc. Grass. Sci., 37, 150-156.

Matyssek, R., and E.-D. Schulze, 1987: Heterosys in hybrid larch (Larix decidua x leptolepsis). I. The role of leaf characteristics. Trees, 1, 219-224.

McClaugherty, C. A., J. D. Aber, and J. M. Melillo, 1982: The role of fine roots in the organic matter and nitrogen budgets of two forested ecosystems. Ecology, 63, 1481-1490. 


\section{Earth Interactions - Volume 4 (2000) - Paper No. 3 - Page 74}

McClaugherty, C. A., J. D. Aber, and J. M. Melillo, 1984: Decomposition dynamics of fine roots in forested ecosystems. Oikos, 42, 378-386.

McClaugherty, C. A., J. Pastor, and J. D. Aber, 1985: Forest litter decomposition in relation to soil nitrogen dynamics and litter quality. Ecology, 66, 266-275.

McWilliam, A.-L. C., J. M. Roberts, O. M. R. Cabral, M. V. B. R. Leitao, A. C. L. de Costa, G. T. Maitelli, and C. A. G. P. Zamparoni, 1993: Leaf area index and above-ground biomass of terra firme rain forest and adjacent clearings in Amazonia. Funct. Ecol., 7, 310-317.

Meinzer, F. C., 1982: The effects of vapor pressure on stomatal control of gas exchange in Douglas fir (Pseudotsuga menziesii) saplings. Oecologia, 54, 236-242.

Melillo, J. M., D. J. Aber, and J. F. Muratore, 1982: Nitrogen and lignin control of hardwood leaf litter decomposition dynamics. Ecology, 63, 621-626.

Menaut, J. C., and J. Cesar, 1979: Structure and primary productivity of Lamto savannas, Ivory Coast. Ecology, 60, 1197-1210.

Milchunas, D. G., and W. K. Laurenroth, 1992: Carbon dynamics and estimates of primary productivity by harvest, ${ }^{14} \mathrm{C}$ dilution, and ${ }^{14} \mathrm{C}$ turnover. Ecology, 73, 593-607.

Miller, H. G., and J. D. Miller, 1976: Effect of nitrogen supply on net primary production in Corsican pine. J. Appl. Ecol., 13, 249-256.

Miller, H. G., J. M. Cooper, and J. D. Miller, 1976: Effect of nitrogen supply on nutrients in litterfall and crown leaching in a stand of Corsican pine. J. Appl. Ecol., 13, 233-248.

Miller, P. M., L. E. Eddleman, and J. M. Miller, 1993: Influence of vapor pressure deficit and soil moisture on gas exchange of Juniperus occidentalis. Northwest Sci., 67, 147-155.

Minderman, G., 1967: Addition, decomposition and accumulation of organic matter in forests. $J$. Ecol., 56, 355-362.

Misra, C. M., 1973: Primary productivity of a grassland ecosystem at Ujjain. Ph.D. dissertation, Vikram University, Ujjain, India. [Available from Vikram University, Ujjain-456010, India.]

Mitchell, A. K., and T. M. Hinckley, 1993: Effects of foliar nitrogen concentration on photosynthesis and water use efficiency in Douglas-fir. Tree Physiol., 12, 403-410.

Miyaji, K.-I., W. S. da Silva, and P. De T. Alvim, 1997: Longevity of leaves of a tropical tree, Theobroma cacao, grown under shading, in relation to position within the canopy and time of emergence. New Phytol., 135, 445-454.

Monsi, D. A., J. G. Isebrands, D. L. Dicmann, and N. N.D., 1953: Über den Lichtfaktor in den pflanzengesellschaften und Seine Bedeutung für die stoff production. Jpn. J. Bot., 29, $17-$ 37.

Monteith, J. L., 1995: A reinterpretation of stomatal responses to humidity. Plant, Cell Environ., 18, 357-364.

Mooney, H. A., and C. Chu, 1983: Stomatal responses to humidity of coastal and interior populations of a California shrub. Oecologia, 57, 148-150.

Morgan, J. A., and R. H. Brown, 1983: Photosynthesis and growth of bermudagrass (Cynodon dactylon cultivar Coastal). Crop Sci., 23, 347-352.

Motovalli, P. P., C. A. Palm, W. J. Parton, E. T. Elliott, and S. D. Frey, 1994: Comparison of laboratory and modeling simulation methods for estimating soil carbon pools in tropical forest soils. Soil Biol. Biochem., 26, 935-944.

Mtambanengwe, F., and H. Kirchmann, 1995: Litter from a tropical savanna woodland (Miombo): Chemical composition and carbon and nitrogen mineralization. Soil Biol. Biochem., 27, $1639-1651$.

Nadelhoffer, K. J., J. D. Aber, and J. M. Melillo, 1985: Fine roots, net primary production, and soil nitrogen availability: A new hypothesis. Ecology, 66, 1377-1390.

Naidu, S. L., J. H. Sullivan, A. H. Teramura, and E. H. DeLucia, 1993: The effects of ultraviolet$B$ radiation on photosynthesis of different aged needles in field-grown loblolly pine. Tree Physiol., 12, 151-162. 
Naik, M. L., 1973: Ecological studies on some grasslands of Ambikapur. Ph.D. dissertation, Sagar University, Sagar, India, [Available from Saugar University, Sagar 470003, India.]

Nambiar, E. K. S., 1987: Do nutrients retranslocate from fine roots. Can. J. For. Res., 17, $917-$ 918.

Nemani, R., S. W. Running, L. E. Band, and D. L. Peterson, 1993: Regional hydroecological simulation system: An illustration of the integration of ecosystem models in a GIS. Environmental Modeling with GIS, edited by M. F. Goodchild, B. O. Parks, and L. T. Steyaert, Oxford University Press, New York, 296-304.

Nemeth, J. C., 1973a: Dry matter production in young loblolly (Pinus taeda L.) and slash pine (Pinus elliottii Engelm.) plantations. Ecol. Monogr., 43, 21-41.

Nemeth, J. C., 1973b: Forest biomass estimation: Permanent plots and regression techniques. IUFRO Biomass Studies, edited by H. E. Young, University of Maine, Orono, ME, 79-88.

Ni, B.-R., and S. G. Pallardy, 1991: Response of gas exchange to water stress in seedlings of woody angiosperms. Tree Physiol., 8, 1-9.

Nihlgard, B., 1972: Plant biomass, primary production and distribution of chemical elements in a beech and a planted spruce forest in south Sweden. Oikos, 23, 69-81.

Nihlgard, B., and L. Lindgren, 1977: Plant biomass, primary production and bio-elements of three mature beech forests in south Sweden. Oikos, 28, 95-104.

Nihlgard, B., and L. Lindgren, 1981: Productivity of forest ecosystems studied during the IBP: The woodlands data set. Dynamic Properties of Forest Ecosystems, edited by D. E. Reichle, Cambridge Univ. Press, New York, 617-621.

Nobel, P. S. 1991: Physiochemical and Environmental Plant Physiology. Academic Press, San Diego, $635 \mathrm{pp}$.

Norman, J. M., and G. S. Campbell, 1989: Canopy structure. Plant Physiological Ecology Field Methods and Instrumentation, edited by R.W. Pearcy et al., Chapman and Hall, London, 301-325.

Oberbauer, S. F., B. R. Strain, and G. H. Reichers, 1987: Field water relations of a wet tropical forest tree species, Pentaclethra macroloba (Mimosaceae). Oecologia, 71, 369-374.

Ogino, K., 1977: A beech forest at Ashiu-Biomass, its increment and net production. Primary Productivity in Japanese Forests, edited by T. Shidei and T. Kira, University of Tokyo Press, Tokyo, 172-186.

Olsvig, L. S., 1980: A comparative study of northeastern pine barrens vegetation. Ph.D. dissertation, Cornell University, Ithaca, NY, 479 pp. [Available from Kroch Library, Cornell University, Ithaca, NY 14853.]

Osonubi, O., and W. J. Davies, 1980: The influence of plant water stress on stomatal control of gas exchange at different levels of atmospheric humidity. Oecologia, 46, 1-6.

Østbye, E., A. Berg, O. Blehr, M. Espeland, E. Gaare, A. Hagen, O. Hesjedal, S. Hagvar, S. Kjelvik, L. Lien, I. Mysterud, A. Sandhaug, H.-K. Skar, A. Skartveit, O. Skre, T. Skogland, T. Solhøy, N. C. Stenseth, and F. E. Wielgolaski, Hardangervidda, 1975: Norway. Structure and Function of Tundra Ecosystems, edited by T. Rosswall and O. W. Heal, Swedish Natural Science Research Council, Stockholm, Sweden, 225-264.

Ovington, J. D., and H. A. I. Madgwick, 1959a: The growth and composition of natural stands of birch. I. Dry matter production. Plant Soil, 10, 271-283.

Ovington, J. D., and H. A. I. Madgwick, 1959b: The growth and composition of natural stands of birch. II. The uptake of nutrients. Plant Soil, 10, 389-400.

Ovington, J. D., D. Heitkamp, and D. B. Lawrence, 1963: Plant biomass and productivity of prairie, savanna, oakwood and maize field ecosystems in central Minnesota. Ecology, 44, $52-63$.

Paavilainen, E., 1980: Effect of fertilization on plant biomass and nutrient cycling on a drained dwarf shrub pine swamp. Comm. Inst. Fenn. For. (Helsinki), Vol. 5, No. 98, Finnish Forest Research Institute, Helsinki, Finland, 71 pp. 
Pan, Y., J. M. Melillo, A. D. McGuire, D. W. Kicklighter, L. F. Pitelka, K. Hibbard, L. L. Pierce, S. W. Running, D. S. Ojima, W. J. Parton, D. S. Schimel, and VEMAP Members, 1998: Modeled responses of terrestrial ecosystems to elevated atmospheric $\mathrm{CO}_{2}: \mathrm{A}$ comparison of simulations by the biogeochemistry models of the Vegetation/Ecosystem Modeling and Analysis Project (VEMAP). Oecologia, 114, 389-404.

Panshin, A. J., and C. de Zeeuw 1980: Textbook of Wood Technology. 4th ed., McGraw-Hill, New York, $722 \mathrm{pp}$.

Panshin, A. J., C. de Zeeuw, and H. P. Brown 1964: Textbook of Wood Technology. 2d ed., McGraw-Hill, New York, 643 pp.

Pastor, J., and J. G. Bockheim, 1981: Biomass and production of an aspen-mixed hardwoodspodosol ecosystem in northern Wisconsin. Can. J. For. Res., 11, 132-138.

Pastor, J., J. D. Aber, and C. A. McClaugherty, 1984: Aboveground production and N and P cycling along a nitrogen mineralization gradient on Blackhawk Island, Wisconsin. Ecology, 65, 256-268.

Pierce, L. L., and S. W. Running, 1988: Rapid estimation of coniferous forest leaf area index using a portable integrating radiometer. Ecology, 69, 1762-1767.

Pierce, L. L., S. W. Running, and J. Walker, 1994: Regional-scale relationships of leaf area index to specific leaf area and leaf nitrogen content. Ecol. Appl., 4, 313-321.

Piper, S. C., 1995: Construction and Description of a Gridded Global Dataset of Daily Temperature and Precipitation for Terrestrial Biospheric Modelling. SIO Reference Series, No. 94-13, Scripps Institute of Oceanography, University of California, San Diego, 73.

Pollard, D. F. W., 1972: Above-ground dry matter production in three stands of trembling aspen. Can. J. For. Res., 2, 27-33.

Poorter, H., and J. R. Evans, 1998: Photosynthetic nitrogen-use efficiency of species that differ inherently in specific leaf area. Oecologia, 116, 26-37.

Potter, C. S., J. T. Randerson, C. B. Field, P. A. Matson, P. M. Vitousek, H. A. Mooney, and S. A. Klooster, 1993: Terrestrial ecosystem production: A process model based on global satellite and surface data. Global Biogeochem. Cycles, 7, 811-841.

Pugnaire, F. I., P. Haase, and J. Puigdefábregas, 1996: Facilitation between higher plant species in a semiarid environment. Ecology, 77, 1420-1426.

Ralston, C. W., 1973: Annual primary productivity in a loblolly pine plantation. IUFRO Biomass Studies, edited by H. E. Young, University of Maine, Orono, ME, 107-117.

Randerson, J. T., M. V. Thompson, T. J. Conway, I. Y. Fung, and C. B. Field, 1997: The contribution of terrestrial sources and sinks to trends in the seasonal cycle of atmospheric carbon dioxide. Global Biogeochem. Cycles, 11, 535-560.

Reich, P. B., and T. M. Hinckley, 1980: Water relations, soil fertility, and plant nutrient composition of a pygmy oak ecosystem. Ecology, 61, 400-416.

Reich, P. B., B. D. Kloeppel, D. S. Ellsworth, and M. B. Walters, 1995a: Different photosynthesisnitrogen relations in deciduous hardwood and evergreen coniferous tree species. Oecologia, 104, 24-30.

Reich, P. B., T. Koike, S. T. Gower, and A. W. Schoettle, 1995b: Causes and consequences of variation in conifer leaf life-span. Ecophysiology of Conifer Forests, edited by W. K. Smith and T. M. Hinckley, Academic Press, New York, 225-254.

Reich, P. B., M. B. Walters, and D. S. Ellsworth, 1997: From tropics to tundra: Global convergence in plant functioning. Proc. Natl. Acad. Sci. USA, 94, 13,730-13,734.

Reich, P. B., D. S. Ellsworth, M. B. Walters, J. M. Vose, C. Gresham, J. C. Volin, and W. D. Bowman, 1999: Generality of leaf trait relationships: A test across six biomes. Ecology, 80, 1955-1969.

Reichle, D. E., N. T. Edwards, W. F. Harris, and P. Sollins, 1981: Productivity of forest ecosystems studied during the IBP: The woodlands data set. Dynamic Properties of Forest Ecosystems, edited by D. E. Reichle, Cambridge Univ. Press, New York, p. 657. 
Reiners, W. A., 1972: Structure and energetics of three Minnesota forests. Ecology, 42, 71-94.

Reiners, W. A., and N. M. Reiners, 1970: Energy and nutrient dynamics of forest floors in three Minnesota forests. J. Ecol., 58, 497-519.

Richards, J. H., 1981: Ecophysiology of a deciduous timberline tree, Larix lyallii Parl. Ph.D. dissertation, University of Alberta, Edmonton, Canada, 228 pp. [Available from University of Alberta, 114 St.-89 Ave., Edmonton, AB T6G 2M7, Canada.]

Rochow, J. J., 1974a: Estimates of above-ground biomass and primary productivity in a Missouri forest. J. Ecol., 62, 567-577.

Rochow, J. J., 1974b: Litterfall relations in a Missouri forest. Oikos, 25, 80-85.

Rochow, J. J., 1975: Mineral nutrient pool and cycling in a Missouri forest. J. Ecol., 63, 985994.

Roessler, R. G., and R. K. Monson, 1985: Midday depression in net photosynthesis and stomatal conductance in Yucca glauca. Relative contributions of leaf temperature and leaf-to-air water vapor concentration difference. Oecologia, 67, 380-387.

Ross, D. J., and K. R. Tate, 1993: Microbial carbon and N, and respiratory activity, in litter and soil in a southern beech (Nothofagus) forest: Distribution and properties. Soil Biol. Biochem., 25, 477-483.

Running, S. W., 1976: Environmental control of leaf water conductance in conifers. Can. J. For. Res., 6, 104-112.

Running, S. W., 1980: Environmental and physiological control of water flux through Pinus contorta. Can. J. For. Res., 10, 82-91.

Running, S. W., 1994: Testing Forest-BGC ecosystem process simulations across a climatic gradient in Oregon. Ecol. Appl., 4, 238-247.

Running, S. W., and J. C. Coughlan, 1988: A general model of forest ecosystem processes for regional applications. I. Hydrological balance, canopy gas exchange and primary production processes. Ecol. Model., 42, 125-154.

Running, S. W., and S. T. Gower, 1991: FOREST BGC, A general model of forest ecosystem processes for regional applications. II. Dynamic carbon allocation and nitrogen budgets. Tree Physiol., 9, 147-160.

Running, S. W., and R. R. Nemani, 1991: Regional hydrologic and carbon balance responses of forests resulting from potential climate change. Clim. Change, 19, 349-368.

Running, S. W., and E. R. Hunt Jr., 1993: Generalization of a forest ecosystem process model for other biomes, BIOME-BGC, and an application for global-scale models. Scaling Physiological Processes: Leaf to Globe, edited by J. R. Ehleringer and C. B. Field, Academic Press, San Diego, 141-157.

Ryan, M. G., 1991: Effects of climate change on plant respiration. Ecol. Appl., 1, 157-167.

Rydholm, S. A. 1965: Pulping Processes. Wiley, New York, 1269.

Sampson, D. A., and H. L. Allen, 1998: Light attenuation in a 14-year-old loblolly pine stand as influenced by fertilization and irrigation. Trees, 13, 80-87.

Sampson, D. A., and F. W. Smith, 1993: Influence of canopy architecture on light penetration in logepole pine (Pinus contorta var. latifolia) forests. Agric. For. Meteorol., 64, 63-79.

Santantonio, D., R. K. Hermann, and W. S. Overton, 1977: Root biomass studies in forest ecosystems. Pedobiologia, 17, 1-31.

Satoo, T., 1970: A synthesis of studies by the harvest method: Primary production relations in the temperate deciduous forests of Japan. Analysis of Temperate Forest Ecosystems, edited by D. E. Reichle, Springer-Verlag, New York, 55-72.

Satoo, T., 1971: Materials for the study of growth in stands. VIII. Primary production relations in plantations of Norway spruce in Japan. Bull. Tokyo Univ. For., 65, 125-142.

Satoo, T., 1973: Materials for the studies of growth of forest stands. XI. Primary production relations in a young plantation of Abies sachalinensis in Hokkaido. Bull. Tokyo Univ. For., 66, 127-137. 
Satoo, T., 1974: Materials for the studies of growth in stands. IX. Primary production relations in a natural forest of Betula maximowicziana in Hokkaido. Bull. Tokyo Univ. For., 66, 109117.

Satoo, T., R. Kunugi, and A. Kumekawa, 1956: Materials for the studies of growth in stands. III. Amount of leaves and production of wood in an aspen (Populus davidiana) second growth in Hokkaido. Bull. Tokyo Univ. For., 52, 33-51.

Scatena, F. N., 1990: Watershed scale rainfall interception on two forested watersheds in the Luquillo Mountains of Puerto Rico. J. Hydrol., 113, 89-102.

Schaefer, D., Y. Steinberger, and W. G. Whitford, 1985: The failure of nitrogen and lignin control of decomposition in a North American desert. Oecologia, 65, 382-386.

Schimel, D. S., B. H. Braswell, R. McKeown, D. S. Ojima, W. J. Parton, and W. Pulliam, 1996: Climate and nitrogen controls on the geography and timescales of terrestrial biogeochemical cycling. Global Biogeochem. Cycles, 10, 677-692.

Schimel, D. S., J. Melillo, H. Tian, A. D. McGuire, D. Kicklighter, T. Kittel, N. Rosenbloom, S. Running, P. Thornton, D. Ojima, W. Parton, R. Kelly, M. Sykes, R. Neilson, and B. Rizzo, 2000: Contribution of increasing $\mathrm{CO}_{2}$ and climate to carbon storage by ecosystems in the United States. Science, 287, 2004-2006.

Schlesinger, W. H., 1978: Community structure, dynamic and nutrient cycling in the Okefenokee cypress swamp forest. Ecol. Monogr., 48, 43-65.

Schlessinger, W. H., 1985: Decomposition of chaparral shrub foliage. Ecology, 66, 1353-1359.

Schulze, E.-D., O. L. Lange, U. Buschbom, L. Kappen, and M. Evenari, 1972: Stomatal responses to changes in humidity in plants growing in the desert. Planta, 108, 259-270.

Schulze, E.-D., F. M. Kelliher, C. Körner, J. Lloyd, and R. Leuning, 1994: Relationships among maximum stomatal conductance, ecosystem surface conductance, carbon assimilation rate and plant nitrogen nutrition: A global ecology scaling exercise. Annu. Rev. Ecol. Syst., 25, $629-660$.

Seeman, J. R., 1989: Light adaptation/acclimation of photosynthesis and the regulation of ribulose1,5-bisphosphate carboxylase activity in sun and shade plants. Plant Physiol., 91, 379-386.

Shankar, V., K. A. Shankarnarayan, and P. Rai, 1973: Primary productivity, energetics and nutrient cycling in Sehima-Heteropogon grassland. I. Seasonal variation in composition, standing crop and net production. Trop. Ecol., 14, 238-251.

Sims, P. L., and R. T. Coupland, 1979: Producers. Grassland Ecosystems of the World: Analysis of Grasslands and Their Uses, edited by R. T. Coupland, Cambridge Univ. Press, New York, 49-72.

Sinclair, T. R., and K. R. Knoer, 1982: Distribution of photosynthetically active radiation in the canopy of a loblolly pine plantation. J. Appl. Ecol., 19, 183-191.

Singer, F. J., and M. K. Harter, 1996: Comparative effects of elk herbivory and 1988 fires on northern Yellowstone National Park grasslands. Ecol. Appl., 6, 185-199.

Singh, A. K., 1972: Structure and primary net production and mineral contents of two grassland communities of Chakia Hill, Varanasi. Ph.D. dissertation, Banaras Hindu University, Varanasi, India. [Available from Banaras Hindu University, Varanasi-221005, India.]

Singh, J. S., and P. S. Yadava, 1974: Seasonal variation in composition, plant biomass and net primary productivity of a tropical grassland at Kurukshetra, India. Ecol. Monogr., 44, 351375.

Singh, J. S., K. P. Singh, and P. S. Yadava, 1979: Ecosystem synthesis. Grassland Ecosystems of the World: Analysis of Grasslands and Their Uses, edited by R. T. Coupland, Cambridge Univ. Press, New York, 231-239.

Smith, S. D., C. A. Herr, K. L. Leary, and J. M. Piorkowski, 1995: Soil-plant water relations in a Mojave Desert mixed shrub community: A comparison of three geomorphic surfaces. $J$. Arid Environ., 29, 339-351. 
Smith, S. D., R. K. Monson, and J. E. Anderson 1997: Physiological Ecology of North American Desert Plants, Adaptations of Desert Organisms. Springer-Verlag, New York, 286 pp.

Smith, T. M., H. H. Shugart, and F. I. Woodward, Eds., 1997: Plant Functional Types: Their Relavence to Ecosystem Properties and Global Change. Cambridge Univ. Press, New York, 369 pp.

Sollins, P., D. E. Reichle, and J. S. Olson, 1973: Organic matter budget and model for a southern Applachian Liriodendron forest. Rep. EDFP-IBP-73/2, Oak Ridge National Laboratory, Oak Ridge, TN.

Staaf, H., and B. Berg, 1982: Accumulation and release of plant nutrients in decomposing Scots pine needle litter. Long-term decomposition in a Scots pine forest II. Can. J. Bot., 60, 15611568.

Stohlgren, T. J., 1988: Litter dynamics in two Sierran mixed conifer forests. II. Nutrient release in decomposing leaf litter. Can. J. For. Res., 18, 1136-1144.

Strojan, C. J., 1978: Forest leaf litter decomposition in the vicinity of a zinc smelter. Oecologia, 32, 203-212.

Stump, L. M., and D. Binkley, 1993: Relationships between litter quality and nitrogen availability in Rocky Mountain forests. Can. J. For. Res., 23, 492-502.

Sugiyama, S., M. Yoneyama, N. Takahashi, and K. Gotoh, 1985: Canopy structure and productivity of Festuca arundinacea Schreb. swards during vegetative and reproductive growth. Grass Forage Sci., 40, 49-55.

Swank, W. T., and H. T. Schreuder, 1973: Temporal changes in biomass, surface area and net production for a Pinus strobus L. forest. IUFRO Biomass Studies, edited by H. E. Young, University of Maine, Orono, ME, 173-182.

Swank, W. T., and H. T. Schreuder, 1974: Comparison of three methods of estimating surface area and biomass for a forest of young eastern white pine. For. Sci., 20, 91-100.

Symbula, M., and F. P. J. Day, 1988: Evaluation of two methods for estimating belowground production in a fresh-water swamp forest. Am. Midl. Nat., 120, 405-415.

Tadaki, Y., K. Hatiya, and H. Miyauchi, 1967: Studies on the productivity of Abies veitchii in the natural forests at Mount Fuji. J. Jpn. For. Soc., 49, 421-428.

Tadaki, Y., K. Hatayi, and K. Tachaiki, 1969: Studies on the production structure of forest. XV. Primary productivity of Fagus crenata in plantation. J. Jpn. For. Soc., 51, 331-339.

Tadaki, Y., K. Hatiya, K. Tochiaki, H. Miyauchi, and U. Matsuda, 1970: Studies on the production structure of forest. XVI. Primary productivity of Abies veitchii in the subalpine zone of Mount Fuji. Bull. Govt. For. Exp. Stn. Tokyo, 229, 1-22.

Taylor, B. R., C. E. Prescott, W. J. F. Parsons, and D. Parkinson, 1991: Substrate control of litter decomposition in four Rocky Mountain coniferous forests. Can. J. Bot., 69, 2242-2250.

Tenhunen, J. D., O. L. Lange, and D. Jahner, 1982: The control by atmospheric factors and water stress of midday stomatal closure in Arbutus unedo growing in a natural macchia. Oecologia, 55, 165-169.

Tenhunen, J. D., R. Hanano, M. Abril, and E. W. Weiler, 1994: Above- and below-ground environmental influences on leaf conductance of Ceanothus thyrsiflorus growing in a chaparral environment: Drought response and the role of abscissic acid. Oecologia, 99, 306-314.

Thornton, P. E., 1998: Description of a numerical simulation model for predicting the dynamics of energy, water, carbon, and nitrogen in a terrestrial ecosystem. Ph.D. dissertation, University of Montana, Missoula, MT, 280 pp. [Available from Mansfield Library, University of Montana, Missoula, MT 59812.]

Thornton, P. E., and S. W. Running, 1999: An improved algorithm for estimating incident daily solar radiation from measurements of temperature, humidity, and precipitation. Agric. For. Meteorol., 93, 211-228.

Thornton, P. E., S. W. Running, and M. A. White, 1997: Generating surfaces of daily meteorological variables over large regions of complex terrain. J. Hydrol., 190, 214-251. 


\section{Earth Interactions - Volume 4 (2000) - Paper No. 3 - Page 80}

Thurnbull, M. H., D. T. Tissue, K. L. Griffin, G. N. D. Rogers, and D. Whitehead, 1998: Photosynthetic acclimation to long-term exposure to elevated $\mathrm{CO}_{2}$ concentration in Pinus radiata D.Don is related to age of needles. Plant, Cell Environ., 21, 1019-1028.

Tieszen, L. L., M. C. Lewis, P. C. Miller, J. Mayo, F. S. Chapin III, and W. C. Oechel, 1981: Tundra ecosystems: A comparative analysis. The International Biological Programme, edited by L. C. Bliss, O. W. Heal, and J. J. Moore, Vol. 25, Cambridge Univ. Press, New York, 285-356.

Timmell, T. E., 1957: Carbohydrate reserves of ten Northern American species of wood. Tappi, 40, 568-572.

Timmell, T. E., 1967: Recent progress in the chemistry of wood hemicellulose. Wood Sci. Technol., 1, 45-70.

Tissue, D. T., K. L. Griffin, R. B. Thomas, and B. R. Strain, 1995: Effects of low and elevated $\mathrm{CO}_{2}$ on $\mathrm{C}_{3}$ and $\mathrm{C}_{4}$ annuals. II. Photosynthesis and leaf biochemistry. Oecologia, 101, 2128.

Titlyanova, A. A., and N. I. Bazilevich, 1979: Ecosystem synthesis of meadows: Nutrient cycling. Grassland Ecosystems of the World, edited by R. T. Coupland, Cambridge Univ. Press, New York, 170-180.

Turner, J., 1981: Nutrient cycling in an age sequence of western Washington Douglas fir stands. Ann. Bot., 48, 159-169.

Turner, J., and J. N. Long, 1975: Accumulation of organic matter in a series of Douglas fir stands. Can. J. For. Res., 5, 681-690.

Turner, J., D. W. Cole, and S. P. Gessel, 1976: Mineral nutrition accumulation and cycling in a stand of red alder (Alnus rubra). J. Ecol., 64, 965-974.

Turner, N. C., E.-D. Schulze, and T. Gollan, 1985: The responses of stomata and leaf gas exchange to vapour pressure deficits and soil water content $\Pi$. In the mesophytic herbaceous species Helianthus annuus. Oecologia, 65, 348-355.

van Cleve, K., L. A. Viereck, and R. L. Schlentner, 1971: Accumulation of nitrogen in alder (Alnus) ecosystems near Fairbanks, Alaska. Arct. Alp. Res., 3, 101-114.

van der Drift, J., 1974: Project Meerdink: Production and decomposition of organic matter in an oak woodland. Final Report 1966-71 of the Netherlands Contribution to the IBP, NorthHolland, New York.

van der Drift, J., 1981: Productivity of forest ecosystems studied during the IBP: The woodlands data set. Dynamic Properties of Forest Ecosystems, edited by D. E. Reichle, Cambridge Univ. Press, New York, p. 607.

van Praag, J. J., S. Sougnez-Remy, F. Weissen, and G. Carletti, 1988: Root turnover in a beech and a spruce stand of the Belgian Ardennes. Plant Soil, 105, 87-103.

VEMAP, 1995: Vegetation/Ecosystem Modeling and Analysis Project (VEMAP): Comparing biogeography and biogeochemistry models in a continental-scale study of terrestrial ecosystem responses to climate change and $\mathrm{CO}_{2}$ doubling. Global Biogeochem. Cycles, 9, 407-437.

Vogt, K. A., C. C. Grier, C. E. Meier, and R. L. Edmonds, 1982: Mycorrhizal role in net primary production and nutrient cycling in Abies amabilis ecosystems in western Washington. Ecology, 63, 370-380.

Vogt, K. A., C. C. Grier, and D. J. Vogt, 1986: Production, turnover, and nutrient dynamics of above- and below-ground detritus of world forests. Adv. Ecol. Res., 15, 303-377.

Vogt, K. A., D. J. Vogt, S. T. Gower, and C. C. Grier, 1990: Carbon and nitrogen interactions for forest ecosystems. Proceedings, Above- and Below-ground Interactions in Forest Trees in Acidified Soils, H. Persson, Ed., Air Pollution Research Report Series, No. 32, Environmental Research Programme of the Commission of the European Communities, 203-235.

Wallin, K. F., and K. F. Raffa, 1999: Altered constitutive and inducible phloem monoterpenes following natural defoliation of jack pine: Implications to host mediated interguild interactions and plant defense theories. J. Chem. Ecol., 25, 861-880. 


\section{Earth Interactions - Volume 4 (2000) - Paper No. 3 • Page 81}

Waring, R. H., and W. H. Schlesinger 1985: Forest Ecosystems: Concepts and Management. Academic Press, San Diego.

Wedin, D. A., L. L. Tieszen, B. Dewey, and J. Pastor, 1995: Carbon isotope dynamics during grass decomposition and soil organic matter formation. Ecology, 76, 1383-1392.

Wells, C. G., J. R. Jorgensen, and C. E. Burnette, 1975: Biomass and mineral elements in a thinned loblolly pine plantation at age 16. USDA Forest Service Research Paper SE-126, Asheville, NC, $10 \mathrm{pp}$.

White, M. A., P. E. Thornton, and S. W. Running, 1997: A continental phenology model for monitoring vegetation responses to interannual climatic variability. Global Biogeochem. Cycles, 11, 217-234.

White, M. A., S. W. Running, and P. E. Thornton, 1999: The impact of growing-season length variability on carbon assimilation and evapotranspiration over 88 years in the eastern U.S. deciduous forest. Int. J. Biometeorol., 42, 139-145.

Whittaker, R. H., 1963: Net production of heath balds and forest heaths in the Great Smoky Mountains. Ecology, 46, 176-182.

Whittaker, R. H., 1966: Forest dimensions and production the Great Smoky Mountains. Ecology, 47, 103-121.

Whittaker, R. H., 1971: Measurement of net primary production of forests. Productivity of Forest Ecosystems, edited by P. Duvigneaud, UNESCO, Paris, 159-175.

Whittaker, R. H., and W. A. Niering, 1968: Vegetation of the Santa Catalina Mountains, Arizona. IV. Limestone and acid soils. J. Ecology, 56, 523-544.

Whittaker, R. H., and W. A. Niering, 1975: Vegetation of the Santa Catalina Mountains, Arizona. V. Biomass, production, and diversity along the elevational gradient. Ecology, 56, 771-790.

Whittaker, R. H., F. H. Bormann, G. E. Likens, and T. G. Siccama, 1974: The Hubbard Brook ecosystem study: Forest biomass and production. Ecol. Monogr., 44, 233-252.

Wielgolaski, F. E., 1975: Primary productivity of alpine meadow communities. Fennoscandian Tundra Ecosystems. Part I: Plants and Microorganisms, edited by F. E. Wielgolaski, Springer-Verlag, New York, 121-128.

Williams, D. G., and R. A. Black, 1994: Drought response of a native and introduced Hawaiian grass. Oecologia, 97, 512-519.

Wise, L. E. and E. C. Jahn, Eds. 1952: Wood Chemistry. 2d ed., Van Nostrand Reinhold, New York.

Woodrow, I. E., and J. A. Berry, 1988: Enzymatic regulation of photosynthetic $\mathrm{CO}_{2}$ fixation in $\mathrm{C}_{3}$ plants. Annu. Rev. Plant Physiol. Plant Molecular Biol., 39, 533-594.

Wullschleger, S. D., 1993: Biochemical limitations to carbon assimilation in $\mathrm{C}_{3}$ plants-A retrospective analysis of the $\mathrm{A} / \mathrm{C}_{\mathrm{i}}$ curves from 109 species. J. Exp. Bot., 44, 907-920.

Yin, X., 1989: Biomass and nutrient fluxes on disturbed and undisturbed sites at the Hardies Creek Forest, Wisconsin. Ph.D. dissertation, University of Minnesota, St. Paul, MN, 169 pp. [Available from University of Minnesota, B-50 Natural Resources, Administration Building, 2003 Upper Buford Circle, St. Paul, MN 55108.].

Yin, X., 1993: Variation in foliar nitrogen concentration by forest type and climatic gradients in North America. Can. J. For. Res., 23, 1587-1602.

Yin, X., and J. A. Perry, 1991: Factors affecting nitrogen concentration of fine roots in forest communities: Regression analysis literature data. For. Sci., 37, 374-382.

Yoshimura, K., 1967: Growth and biomass of Norway spruce forest in Ashu experimental forest. Bull. Kyoto Univ. For., 39, $27-34$.

Young, H. E., 1972: Biomass sampling methods for puckerbush studies. in Forest Biomass Studies, IUFRO Working Group on Forest Biomass Studies, University of Maine, Orono, ME, 179190.

Zavitkovski, J., and R. D. Stevens, 1972: Primary productivity of red alder ecosystems. Ecology, 53, 235-242. 
Zavitkovski, J., J. G. Isebrands, and T. R. Crow, 1976: Application of growth analysis in forest biomass studies.. Proceedings of the Third North American Forest Biology Workshop, C. P. P. Reid and C. H. Fechner, Eds., College of Forestry and Natural Resources, Colorado State University, Fort Collins, CO, 196-226.

Zimmermann, P. H., J. Feichter, H. K. Rath, P. J. Crutzen, and W. Weiss, 1989: A global threedimensional source-receptor model investigation using ${ }^{85} \mathrm{Kr}$. Atmos. Environ., 23, 25-35.

Zobler, L., 1986: A world soil file for global climate modeling. NASA Tech. Memo. 87802, 33 pp.

Table 1. Mean BIOME-BGC parameter values for evergreen needle leaf forest (ENF), shrub, deciduous needle leaf forest (DNF), deciduous broadleaf forest (DBF), and grass biomes. Parameter descriptions and individual values are presented in appendix A.

\begin{tabular}{|c|c|c|c|c|c|}
\hline $\begin{array}{l}\text { Parameter } \\
(\mathrm{C}=\text { carbon, } \mathrm{N}=\text { nitrogen })\end{array}$ & $\mathrm{ENF}$ & Shrub & DNF & $\mathrm{DBF}$ & Grass \\
\hline Leaf and fine root turnover (LFRT) ${ }^{\mathrm{a}}$ & 0.26 & 0.26 & 1.0 & 1.0 & 1.0 \\
\hline Live wood turnover $(\text { LWT })^{a}$ & 0.70 & 0.70 & 0.70 & 0.70 & \\
\hline Fire mortality $(\mathrm{FM})^{\mathrm{a}}$ & $5.0 \mathrm{E}-3$ & 0.010 & $5.0 \mathrm{E}-3$ & $2.5 \mathrm{E}-3$ & $5.0 \mathrm{E}-2$ \\
\hline Whole plant mortality (WPM) ${ }^{\mathrm{a}}$ & $5.0 \mathrm{E}-3$ & 0.020 & $5.0 \mathrm{E}-3$ & $5.0 \mathrm{E}-3$ & 0.10 \\
\hline New fine root $C$ to new leaf $C$ ratio $(F R C: L C)^{b}$ & 1.4 & 1.4 & 1.4 & 1.2 & 1.0 \\
\hline New stem $C$ to new leaf $C$ ratio $(S C: L C)^{b}$ & 2.2 & 0.22 & 2.2 & 2.2 & \\
\hline $\begin{array}{l}\text { New live wood } C \text { to new total wood } C \text { ratio } \\
\text { (LWC:TWC })^{b}\end{array}$ & 0.071 & 1.0 & 0.071 & 0.16 & \\
\hline New coarse root $C$ to new stem $C$ ratio $(C R C: S C)^{b}$ & 0.29 & 0.29 & 0.29 & 0.22 & \\
\hline Leaf $C: N\left(C: N_{\text {leaf }}\right)^{c}$ & 42 & 35 & 27 & 25 & 25 \\
\hline Litter $C: N\left(C: \mathrm{N}_{\mathrm{lit}}\right)^{\mathrm{c}}$ & 93 & 75 & 120 & 55 & 45 \\
\hline Fine root $C: N\left(C: N_{f r}\right)^{c}$ & 58 & 58 & 58 & 48 & 50 \\
\hline Live wood C:N $\left(C: N_{1 w}\right)^{c}$ & 50 & 50 & 50 & 50 & \\
\hline Dead wood C:N $\left(C: N_{d w}\right) c$ & 730 & 730 & 730 & 550 & \\
\hline Fine root labile $\left(\mathrm{FR}_{\mathrm{lab}}\right)^{\mathrm{d}}$ & 34 & 34 & 34 & 34 & 34 \\
\hline Fine root cellulose $\left(\mathrm{FR}_{\mathrm{cec}}\right)^{\mathrm{d}}$ & 44 & 44 & 44 & 44 & 44 \\
\hline Fine root lignin $\left(\mathrm{FR}_{\mathrm{lig}}\right)^{\mathrm{d}}$ & 22 & 22 & 22 & 22 & 22 \\
\hline Litter labile $\left(\mathrm{L}_{\mathrm{lab}}\right)^{\mathrm{d}}$ & 31 & 56 & 31 & 38 & 68 \\
\hline Litter cellulose $\left(\mathrm{L}_{\text {cel }}\right)^{\mathrm{d}}$ & 45 & 29 & 45 & 44 & 23 \\
\hline Litter lignin $\left(\mathrm{L}_{\mathrm{iig}}\right)^{\mathrm{d}}$ & 24 & 15 & 24 & 18 & 9.0 \\
\hline Dead wood cellulose $\left(\mathrm{DW}_{\mathrm{cel}}\right)^{\mathrm{d}}$ & 71 & 71 & 71 & 77 & \\
\hline Dead wood lignin $\left(\mathrm{DW}_{\mathrm{lig}}\right)^{\mathrm{d}}$ & 29 & 29 & 29 & 23 & \\
\hline Specific leaf area (SLA)e & 8.2 & 12 & 22 & 32 & 49 \\
\hline All-sided to projected leaf area ratio $\left(\mathrm{LAI}_{\text {allproro }}\right)^{\mathrm{f}}$ & 2.6 & 2.3 & 2.6 & 2.0 & 2.0 \\
\hline Shaded to sunlit specific leaf area ratio $\left(\mathrm{SLA}_{\text {shd:sun }}\right)^{\mathrm{g}}$ & 2.0 & 2.0 & 2.0 & 2.0 & 2.0 \\
\hline Maximum stomatal conductance $\left(g_{s \max }\right)^{\mathrm{h}}$ & $6.0 \mathrm{E}-3$ & $6.0 \mathrm{E}-3$ & $6.0 \mathrm{E}-3$ & $6.0 \mathrm{E}-3$ & $6.0 \mathrm{E}-3$ \\
\hline Cuticular conductance $\left(g_{\mathrm{cut}}\right)^{\mathrm{h}}$ & $6.0 \mathrm{E}-5$ & $6.0 \mathrm{E}-5$ & $6.0 \mathrm{E}-5$ & $6.0 \mathrm{E}-5$ & $6.0 \mathrm{E}-5$ \\
\hline Boundary layer conductance $\left(g_{\mathrm{b}}\right)^{\mathrm{h}}$ & 0.09 & 0.02 & 0.09 & 0.01 & 0.04 \\
\hline $\begin{array}{l}\text { Leaf water potential at initial } g_{s \max } \text { reduction } \\
\left(\mathrm{LWP}_{\mathrm{i}}\right)^{\mathrm{i}}\end{array}$ & -0.65 & -0.81 & -0.65 & -0.34 & -0.73 \\
\hline $\begin{array}{l}\text { Leaf water potential at final } g_{s \max } \text { reduction } \\
\left(\mathrm{LWP}_{\mathrm{f}}\right)^{\mathrm{i}}\end{array}$ & -2.5 & -4.2 & -2.5 & -2.2 & -2.7 \\
\hline $\begin{array}{l}\text { Vapor pressure deficit at initial } g_{\text {smax }} \text { reduction } \\
\left(\text { VPD }_{\mathrm{i}}\right)^{\mathrm{j}}\end{array}$ & 0.61 & 0.97 & 0.61 & 1.1 & 1.0 \\
\hline $\begin{array}{l}\text { Vapor pressure deficit at final } g_{s \max } \text { reduction } \\
\left(\mathrm{VPD}_{\mathrm{f}}\right)^{j}\end{array}$ & 3.1 & 4.1 & 3.1 & 3.6 & 5.0 \\
\hline Water interception coefficient $\left(W_{\text {int }}\right)^{\mathrm{k}}$ & 0.045 & 0.045 & 0.045 & 0.045 & 0.022 \\
\hline Light extinction coefficient $(\mathrm{k})^{1}$ & 0.51 & 0.55 & 0.51 & 0.54 & 0.48 \\
\hline Percent of leaf $\mathrm{N}$ in Rubisco (PLNR) ${ }^{\mathrm{d}}$ & 3.3 & 3.3 & 8.8 & 8.8 & 21 \\
\hline
\end{tabular}

Unit key: ${ }^{\mathrm{a}}=1 \mathrm{yr}^{-1}, \mathrm{~b}=\mathrm{kg} \mathrm{C} \mathrm{kg} \mathrm{C}^{-1},{ }^{\mathrm{c}}=\mathrm{kgC} \mathrm{kg} \mathrm{N}{ }^{1},{ }^{d}=$ percent, ${ }^{\mathrm{e}}=\mathrm{m}^{2} \mathrm{~kg} \mathrm{C}^{-1},{ }^{\mathrm{f}}=\mathrm{LAI} \mathrm{LAI}^{-1},{ }^{\mathrm{g}}=$ SLA SLA ${ }^{-1},{ }^{\mathrm{h}}=\mathrm{m} \mathrm{s}^{-1},{ }^{\mathrm{i}}=\mathrm{MPa},{ }^{\mathrm{j}}=\mathrm{kPa},{ }^{\mathrm{k}}=1 \mathrm{LAI}^{-1}$ day $^{-1},{ }^{1}=$ unitless. 
Table 2. Effect of varying input parameters in evergreen needle leaf (ENF), shrub, deciduous needle leaf (DNF), deciduous broad leaf (DBF), $C_{3}$ grass (C3G), and $C_{4}$ grass (C4G) biomes. Here, $\Delta=$ change in simulated mean annual 1895-1993 net primary production (NPP, $\mathrm{g} \mathrm{m}^{-2}$ ) caused by increasing the parameter from the mean minus $20 \%$ to the mean plus $20 \%$. Each value represents the mean from 10 pixels per biome. Only those parameters significantly affecting at least one biome are shown. LFRT not varied for deciduous biomes.

\begin{tabular}{lllllll}
\hline & ENF & Shrub & DNF & DBF & C3G & C4G \\
\hline C:N $_{\text {lear }}$ & $-87^{\mathrm{a}}$ & $-24^{\mathrm{a}}$ & $-80^{\mathrm{a}}$ & $-63^{\mathrm{a}}$ & $31^{\mathrm{a}}$ & $36^{\mathrm{a}}$ \\
C:N & $72^{\mathrm{a}}$ & $19^{\mathrm{a}}$ & $24^{\mathrm{c}}$ & $42^{\mathrm{a}}$ & $26^{\mathrm{a}}$ & $30^{\mathrm{a}}$ \\
LFRT & $43^{\mathrm{a}}$ & $4^{\mathrm{c}}$ & & & & \\
FRC:LC & $-56^{\mathrm{a}}$ & $-16^{\mathrm{a}}$ & $-48^{\mathrm{a}}$ & $-18^{\mathrm{c}}$ & $6^{\mathrm{c}}$ & $8^{\mathrm{b}}$ \\
SLA & $-64^{\mathrm{a}}$ & $-11^{\mathrm{a}}$ & $-29^{\mathrm{b}}$ & $-62^{\mathrm{a}}$ & $-5^{\mathrm{c}}$ & $-2^{\mathrm{c}}$ \\
$\mathrm{g}_{\text {smax }}$ & $-61^{\mathrm{a}}$ & $-14^{\mathrm{a}}$ & $-45^{\mathrm{a}}$ & $-38^{\mathrm{a}}$ & $-7^{\mathrm{b}}$ & $1^{\mathrm{c}}$ \\
$\mathrm{LAI}_{\text {all:proj }}$ & $-41^{\mathrm{a}}$ & $-2^{\mathrm{c}}$ & $0^{\mathrm{c}}$ & $0^{\mathrm{c}}$ & $0^{\mathrm{c}}$ & $0^{\mathrm{c}}$ \\
$\mathrm{PLNR}^{\mathrm{c}}$ & $142^{\mathrm{a}}$ & $37^{\mathrm{a}}$ & $104^{\mathrm{a}}$ & $104^{\mathrm{a}}$ & $4^{\mathrm{c}}$ & $-1^{\mathrm{c}}$ \\
$\mathrm{FR}_{\text {cel }}$ & $0^{\mathrm{c}}$ & $0^{\mathrm{c}}$ & $21^{\mathrm{c}}$ & $5^{\mathrm{c}}$ & $27^{\mathrm{a}}$ & $32^{\mathrm{a}}$ \\
$\mathrm{FR}_{\text {lig }}$ & $0^{\mathrm{c}}$ & $0^{\mathrm{c}}$ & $-19^{\mathrm{c}}$ & $-5^{\mathrm{c}}$ & $-21^{\mathrm{a}}$ & $-27^{\mathrm{a}}$ \\
$W_{\text {int }}$ & $-41^{\mathrm{a}}$ & $-2^{\mathrm{c}}$ & $-16^{\mathrm{c}}$ & $-31^{\mathrm{b}}$ & $-1^{\mathrm{c}}$ & $0^{\mathrm{c}}$ \\
$\mathrm{K}$ & $7^{\mathrm{c}}$ & $-3^{\mathrm{c}}$ & $17^{\mathrm{c}}$ & $-54^{\mathrm{a}}$ & $0^{\mathrm{c}}$ & $-2^{\mathrm{c}}$ \\
$\mathrm{FR}_{\text {lab }}$ & $0^{\mathrm{c}}$ & $0^{\mathrm{c}}$ & $15^{\mathrm{c}}$ & $2^{\mathrm{c}}$ & $13^{\mathrm{a}}$ & $14^{\mathrm{a}}$ \\
$\mathrm{L}_{\text {cel }}$ & $0^{\mathrm{c}}$ & $0^{\mathrm{c}}$ & $9^{\mathrm{c}}$ & $4^{\mathrm{c}}$ & $10^{\mathrm{a}}$ & $13^{\mathrm{a}}$ \\
$\mathrm{FM}^{\mathrm{c}}$ & $-\mathrm{l}^{\mathrm{c}}$ & $0^{\mathrm{c}}$ & $-23^{\mathrm{c}}$ & $-3^{\mathrm{c}}$ & $-58^{\mathrm{a}}$ & $-73^{\mathrm{a}}$ \\
$\mathrm{L}_{\text {lab }}$ & $0^{\mathrm{c}}$ & $0^{\mathrm{c}}$ & $4^{\mathrm{c}}$ & $1^{\mathrm{c}}$ & $16^{\mathrm{a}}$ & $17^{\mathrm{a}}$ \\
\hline
\end{tabular}

a Significant at the $1 \%$ level.

b Significant at the $5 \%$ level.

c Not significant.

Table 3. Design of the $2^{5-1}$ half-fraction factorial sensitivity analysis for ENF and C3G biomes. Columns two-six show contrast coefficients for the five parameters in the analysis (different for ENF and C3G). A plus symbol indicates that the parameter was set at the mean plus the standard error while a minus symbol indicates mean minus standard error. When C3G FR $L_{c e l}$ were set to,$+ F_{l l g}$ and $L_{\text {lig }}$ were reduced by equal magnifude; thus $+F R_{c e l}$ and $+L_{c e l}$ indicate high quality litter and $-F_{\text {cel }}$ and $-L_{c e l}$ indicate the reverse. The ENF and C3G columns under the mean NPP section show the mean NPP $\left(\mathrm{g} \mathrm{m}^{-2}\right.$ ) from the simulation using the parameter levels of columns two-six. For example, simulation 1 had C: $\mathrm{N}_{\text {leat }}, g_{\text {smax }}, \mathrm{PLNR}$, and SLA at low levels and $\mathrm{C}: \mathrm{N}_{\mathrm{fr}}$ at a high level and produced a mean 99-yr NPP for the 10 ENF pixels of $441 \mathrm{~g} \mathrm{~m}^{-2}$.

\begin{tabular}{|c|c|c|c|c|c|c|c|}
\hline \multicolumn{6}{|c|}{ Parameter contrast coefficient } & \multicolumn{2}{|c|}{ Mean NPP } \\
\hline $\begin{array}{l}\text { ENF } \\
\text { C3G }\end{array}$ & $\begin{array}{l}C: N_{\text {leaf }} \\
C: N_{\text {leaf }}\end{array}$ & $\begin{array}{c}g_{\operatorname{smax}} \\
\text { FM }\end{array}$ & $\begin{array}{c}\text { PLNR } \\
\text { FR }_{\text {cel }}\end{array}$ & $\begin{array}{c}\text { SLA } \\
\mathrm{L}_{\text {cel }}\end{array}$ & $\begin{array}{l}C: N_{f r} \\
C: N_{f r}\end{array}$ & ENF & $\mathrm{C} 3 \mathrm{G}$ \\
\hline 1 & - & - & - & - & + & 441 & 228 \\
\hline 2 & + & - & - & - & - & 375 & 220 \\
\hline 3 & - & + & - & - & - & 388 & 193 \\
\hline 4 & + & + & - & - & + & 400 & 212 \\
\hline 5 & - & - & + & - & - & 472 & 229 \\
\hline
\end{tabular}


Earth Interactions - Volume 4 (2000) - Paper No. 3 • Page 84

Table 3. (Continued)

\begin{tabular}{|c|c|c|c|c|c|c|c|}
\hline \multicolumn{6}{|c|}{ Parameter contrast coefficient } & \multicolumn{2}{|c|}{ Mean NPP } \\
\hline $\begin{array}{l}\text { ENF } \\
\text { C3G } \\
\end{array}$ & $\begin{array}{l}C: N_{\text {leaf }} \\
C: N_{\text {leaf }}\end{array}$ & $\begin{array}{c}g_{\text {smax }} \\
\mathrm{FM} \\
\end{array}$ & $\begin{array}{c}\text { PLNR } \\
\text { FR }_{\text {cel }} \\
\end{array}$ & $\begin{array}{l}\text { SLA } \\
\mathrm{L}_{\text {cel }}\end{array}$ & $\begin{array}{l}C: N_{f r} \\
C: N_{f r}\end{array}$ & ENF & C3G \\
\hline 6 & + & - & + & - & + & 493 & 247 \\
\hline 7 & - & + & + & - & + & 498 & 218 \\
\hline 8 & + & + & + & - & - & 439 & 216 \\
\hline 9 & - & - & - & + & - & 381 & 225 \\
\hline 10 & + & - & - & + & + & 392 & 245 \\
\hline 11 & - & + & - & + & + & 403 & 217 \\
\hline 12 & + & + & - & + & - & 342 & 209 \\
\hline 13 & - & - & + & + & + & 487 & 253 \\
\hline 14 & + & - & + & + & - & 430 & 245 \\
\hline 15 & - & + & + & + & - & 437 & 221 \\
\hline 16 & + & + & + & + & + & 453 & 241 \\
\hline
\end{tabular}

Table 4. Main effects of parameter variation in ENF and C3G biomes. Effect columns show the expected effect on NPP $\left(\mathrm{g} \mathrm{m}^{-2}\right)$ of raising a parameter from the mean minus standard error to the mean plus standard error. Values are mean and $95 \%$ confidence interval calculated from the $t$ distribution with nine degrees of freedom.

\begin{tabular}{|c|c|c|c|}
\hline \multicolumn{2}{|c|}{ ENF } & \multicolumn{2}{|c|}{$\mathrm{C} 3 \mathrm{G}$} \\
\hline Parameter & Effect & Parameter & Effect \\
\hline $\mathrm{C}: \mathrm{N}_{\text {leaf }}$ & $-23.1 \pm 7.9$ & $\mathrm{C}: \mathrm{N}_{\text {leaf }}$ & $6.6 \pm 1.8$ \\
\hline$g_{\operatorname{smax}}$ & $-13.9 \pm 3.6$ & $\mathrm{FM}$ & $-20.6 \pm 3.4$ \\
\hline PLNR & $73.3 \pm 23.1$ & $\mathrm{FR}_{\text {cel }}$ & $15.0 \pm 3.8$ \\
\hline SLA & $-22.6 \pm 7.0$ & $\mathrm{~L}_{\mathrm{cel}}$ & $11.6 \pm 2.9$ \\
\hline$C: N_{f r}$ & $37.9 \pm 14.5$ & $C: N_{f r}$ & $13.0 \pm 3.8$ \\
\hline
\end{tabular}

Table 5. Two-way interaction effects of parameter variation in ENF and C3G biomes. The interaction columns show the expected interaction effect on NPP $\left(\mathrm{g} \mathrm{m}^{-2}\right)$ caused by raising the shown parameters from mean minus standard error to mean plus standard error. Values are mean and $95 \%$ confidence interval calculated from the $t$ distribution with nine degrees of freedom (* indicates significant interaction effect).

\begin{tabular}{|c|c|c|c|}
\hline \multicolumn{2}{|c|}{ ENF } & \multicolumn{2}{|c|}{$\mathrm{C} 3$} \\
\hline Parameters & Interaction & Parameters & Interaction \\
\hline$C: N_{\text {leaf }} \times g_{\text {smax }}$ & $-0.2 \pm 0.9$ & $\mathrm{C}: \mathrm{N}_{\mathrm{leaf}} \times 9.1 .1 .4$ & $0.7 \pm 1.4$ \\
\hline C: $N_{\text {leaf }} \times$ PLNR $^{*}$ & $2.9 \pm 2.5$ & $C: N_{\text {leaf }} \times F R_{c e l}$ & $0.5 \pm 0.9$ \\
\hline$C: \mathrm{N}_{\mathrm{leaf}} \times 9.1 .1 .1 .2$ & $-0.1 \pm 0.5$ & $\mathrm{C}: \mathrm{N}_{\text {leaf }} \times \mathrm{L}_{\mathrm{cel}}$ & $-0.6 \pm 1.1$ \\
\hline $\mathrm{C}: \mathrm{N}_{\text {leaf }} \times \mathrm{C}: \mathrm{N}_{\mathrm{fr}}$ & $0.1 \pm 0.7$ & $\mathrm{C}: \mathrm{N}_{\text {leaf }} \times \mathrm{C}: \mathrm{N}_{\mathrm{fr}}$ & $0.8 \pm 1.1$ \\
\hline$g_{\text {smax }} \times$ PLNR & $0.2 \pm 1.1$ & $\mathrm{FM} \times \mathrm{FR}_{\mathrm{cel}}$ & $1.3 \pm 2.9$ \\
\hline$g_{s \max } \times$ SLA & $0.1 \pm 0.5$ & $\mathrm{FM} \times \mathrm{L}_{\mathrm{cel}}$ & $0.4 \pm 1.4$ \\
\hline$g_{s \operatorname{six}} \times C: \mathbf{N}_{\mathrm{fr}} *$ & $-0.7 \pm 0.2$ & $\mathrm{FM} \times \mathrm{C}: \mathrm{N}_{\mathrm{fr}}$ & $-0.5 \pm 1.1$ \\
\hline PLNR $\times 1.1 .1 .1 .3$ & $-1.0 \pm 1.1$ & $\mathrm{FR}_{\text {cel }} \times \mathrm{L}_{\text {cel }}$ & $0.9 \pm 1.1$ \\
\hline
\end{tabular}


Table 6. Comparison of parameterization schemes. Spatial prediction refers to a mean value calculated from Figure 3 or Figure 4; single value per biome refers to the mean value calculated from the literature searches described in the preceding sections. Parameters are leaf carbon to nitrogen ratio ( $C: \mathrm{N}_{\text {leaf }}, \mathrm{kg} C \mathrm{~kg} \mathrm{~N}^{-1}$ ), specific leaf area (SLA, $\mathrm{m}^{2} \mathrm{~kg} \mathrm{C}^{-1}$ ), leaf life span (months), and the percent of leaf nitrogen in Rubisco (PLNR, dimensionless). Biomes are evergreen needle leaf forest (ENF) and deciduous broad leaf forest (DBF). DBF leaf life span from BIOME-BGC phenology model (White et al. 1997).

\begin{tabular}{lccccc}
\hline & \multicolumn{2}{c}{ ENF } & & \multicolumn{2}{c}{ DBF } \\
\cline { 2 - 3 } \cline { 5 - 6 } & $\begin{array}{c}\text { Spatial } \\
\text { prediction }\end{array}$ & $\begin{array}{c}\text { Single value } \\
\text { per biome }\end{array}$ & & $\begin{array}{c}\text { Spatial } \\
\text { prediction }\end{array}$ & $\begin{array}{c}\text { Single value } \\
\text { per biome }\end{array}$ \\
\hline C: $N_{\text {leaf }}$ & 50 & 42 & & 33 & 25 \\
SLA & 9.2 & 8.2 & & 16 & 32 \\
Leaf life span & 47 & 46 & & 13 & 6.6 \\
PLNR & 3.9 & 3.3 & & 5.5 & 8.8 \\
\hline
\end{tabular}

Earth Interactions is published jointly by the American Meteorological Society, the American Geophysical Union, and the Association of American Geographers. Permission to use figures, tables, and brief excerpts from this journal in scientific and education works is hereby granted provided that the source is acknowledged. Any use of material in this journal that is determined to be "fair use" under Section 107 or that satisfies the conditions specified in Section 108 of the U.S. Copyright Law (17 USC, as revised by P.L. 94-553) does not require the publishers' permission. For permission for any other form of copying, contact one of the copublishing societies. 